\title{
Modification of the Disease Progression in Mice with \\ Experimental Autoimmune Encephalomyelitis Using
}

Anti-Mitotic Compounds.

By

Kevin Patrick Crume

\author{
A thesis submitted to \\ Victoria University of Wellington \\ in fulfilment of the requirements for the degree of \\ Doctor of Philosophy in Cell \& Molecular Bioscience
}

Victoria University of Wellington 


\section{Abstract}

Multiple sclerosis (MS) is a multi-faceted disease, and is believed to be caused by an autoimmune response to myelin antigens in the central nervous system. Experimental autoimmune encephalomyelitis (EAE), an animal model for MS, manifests itself in various forms that parallel many aspects of MS, including the appearance of symptoms, initiation events, and pathophysiology. The hallmark of any immune response is the antigen-specific proliferation of immune cells, and during the initiation events of EAE, proliferating $\mathrm{CD}^{+} \mathrm{T}$ cells are the primary mediators of disease. This thesis explores if targeting these proliferating cells with the anti-mitotic compounds paclitaxel and peloruside A can delay or prevent the onset of EAE, thus providing a novel therapeutic avenue for MS research.

The anti-cancer compound, paclitaxel, is an anti-mitotic drug that prevents microtubule depolymerisation. Although paclitaxel has been used in the clinical setting to treat cancer for over a decade, it has been determined that paclitaxel stimulates murine toll-like receptor 4 (TLR4) complex, which is the major LPS receptor. A novel microtubule-stabilising compound, peloruside, is currently subject to intensive investigations due to its functional similarity to paclitaxel. The results from this project found that peloruside and paclitaxel inhibited the proliferation of mitogen-stimulated splenocytes with $\mathrm{IC}_{50}$ values of $83 \mathrm{nM}$ and $30 \mathrm{nM}$, respectively, but did not affect the viability of non-proliferating cells. In contrast to paclitaxel, peloruside did not cause the TLR4-mediated production of the inflammatory mediators, TNF- $\varepsilon$, IL-12, and nitric oxide, when cultured with IFN- $\varepsilon$ stimulated murine macrophages. Interestingly, when LPS was included with either paclitaxel or 
peloruside A, both drugs decreased the production of TNF- $\varepsilon$ and nitric oxide from macrophages, suggesting that microtubule-stabilising compounds may have antiinflammatory effects.

To identify any immunomodifying effects of paclitaxel in vivo, paclitaxel was administered to mice that were immunised with the myelin protein MOG in complete Freund's adjuvant (CFA) to induce EAE. When Taxol was administered to mice for 5 consecutive days immediately following CFA/MOG immunisation, the onset of EAE was delayed by approximately 1 week. Moreover, the administration of peloruside following the same treatment regime also resulted in a similar delay of disease onset. Taxol treatments, however, lead to significant mortality in immunised, but not unimmunised mice. Interestingly, although Taxol is an anti-mitotic drug, the proliferation of antigen-specific T cells was not inhibited in vivo by the Taxol treatment. The findings revealed in this thesis present an opportunity to pursue a new avenue of research for the therapeutic treatment of MS sufferers, and possibly other inflammatory autoimmune disorders. 


\section{Acknowledgements}

I would especially like to acknowledge the guidance provided by my supervisor, Dr. Anne La Flamme. I couldn't have asked for better supervision, and it has been an honour to be her first PhD student. Also, I can't say thanks enough to my cosupervisor, Dr. John Miller, for providing such excellent feedback, as well as keeping us students smiling.

This work could not have been done without the endless support and encouragement from my wife, Joanne. Other supporters include Marina, Joanna, Paula, Lisa, Thomas Backstrom, and the EAE group at Malaghan Institute of Medical Research. These individuals have been vital for the success of this project. Although the Monday meetings were difficult sometimes, I always felt a bit more smarterer afterwards.

I'd also like to acknowledge the friendship and support of my co-workers in and around KK601. Tom, Andrew, Bruce, James, Marina, Cliff, Simon and Tim: you fellas have made education fun, but you never taught me anything about biology! To Anja, Amy, and Bronwyn: thank you for being tolerant.

Other key individuals include Dr. Peter Northcote and the Natural Products Group in the VUW Chemistry Department for providing peloruside, Dr. Shirley Pledger for her statistical advice, Shay for maintaining the animal facility, the SBS administration, and Sarah and Greg at the Pathology Dept., Wellington Hospital, for preparing slides for histology.

Lastly, this research would have been impossible without the funding from Wellington Medical Research Foundation, VUW Faculty of Science Research Grant, and the VUW Postgraduate Scholarship. 


\section{Table of Contents}

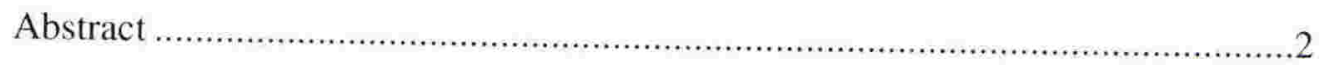

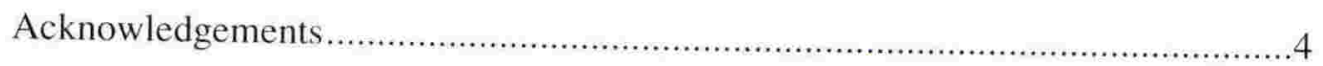

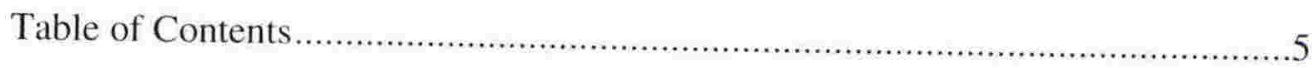

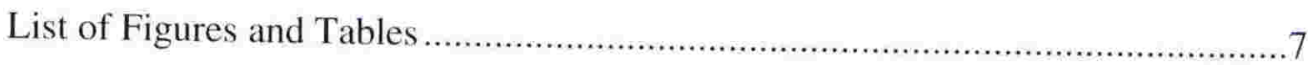

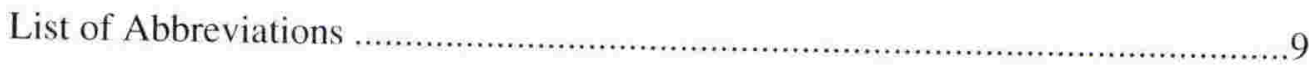

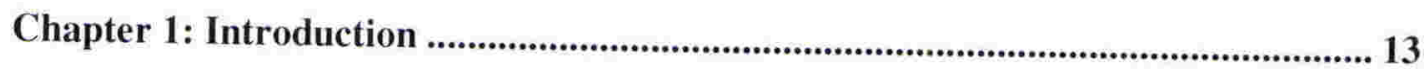

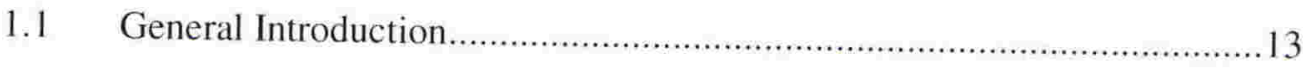

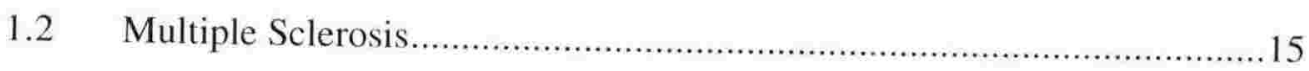

1.3 Experimental Autoimmune Encephalomyelitis ......................................2 24

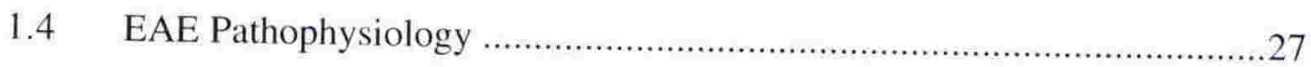

1.5 Current MS Treatments and Investigational Therapies.............................34

1.6 Anti-proliferative Agents and the Treatment of Hyper-proliferative

Diseases

1.7 Aims

Chapter 2: General Methods

2.1 Animals, Maintenance, and Ethical Guidelines ...................................4 44

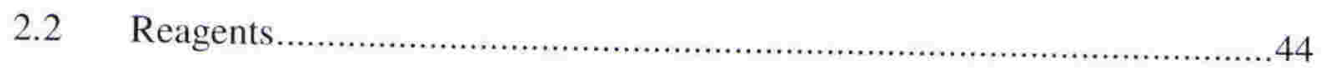

2.3 Primary Cell Preparation and Culture .................................................45

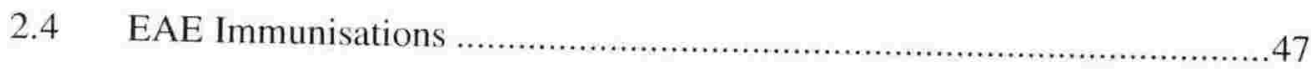

2.5 Measuring Body Temperature, Weight, and Appearance of Mice. ............48

2.6 Enzyme-Linked Immunosorbant Assays for Cytokine Analysis ...............49

2.7 Metabolism and Proliferation Assays.................................................49

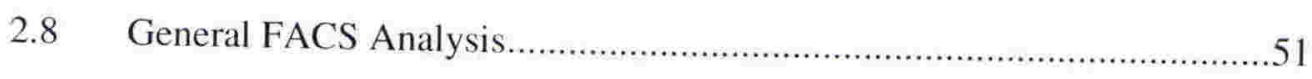

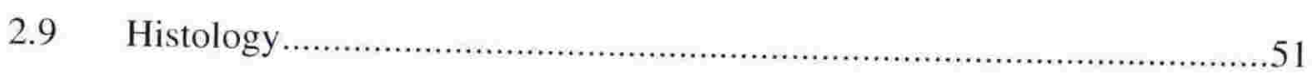


Chapter 3: Immunomodulatory Effects of the Anti-Mitotic Compounds,

Paclitaxel and Peloruside.

3.1 Introduction

3.2 Methods

3.3 Results

3.4 Discussion

Chapter 4: In Vivo Immunomodulatory Effects of Paclitaxel

4.1 Introduction

4.2 Methods

4.3 Results

4.4 Discussion

Chapter 5: Effects of Anti-Mitotic Drugs on EAE.

5.1 Introduction

5.2 Methods

5.3 Results

5.4 Discussion

Chapter 6: General Discussion

6.1 Summary.

6.2 Future Directions

References

Appendix A: $\quad$ Buffers and Reagents ................................................................. 161

Appendix B: Determining peloruside purity .................................................... 164

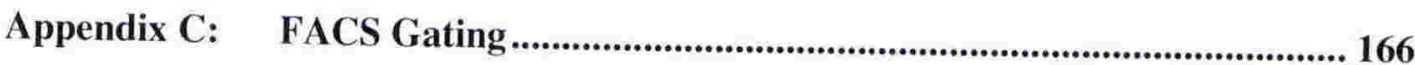




\section{List of Figures and Tables}

\section{Chapter 1}

Figure 1.1 The four subtypes of MS

Figure 1.2 Hematoxylin and eosin (H\&E) stained longitudinal sections of spinal cords.

Figure 1.3 A schematic interpretation of the molecular and pathological events that lead to myelin destruction.

Figure 1.4 Structures of the anti-mitotic, microtubule-stabilising compounds, paclitaxel and peloruside A.

Table 1.1 Current MS therapies.

\section{Chapter 2}

Figure 2.1 Examples of infiltrating cell foci.

\section{Chapter 3}

Figure 3.1 Anti-CD3-stimulated T cell responses to sponge metabolites and cyclosporin A.

Figure 3.2 Determining $\mathrm{IC}_{50}$ values of peloruside and paclitaxel in splenocytes.

Figure 3.3 Effect of peloruside and paclitaxel on BMMØ.

Figure 3.4 Paclitaxel in BMM $\varnothing$.

Figure 3.5 Peloruside in BMMØ.

Figure 3.6 Effect of peloruside and paclitaxel in LPS-stimulated BMMØ.

\section{Chapter 4}

Figure 4.1 Taxol has no effect on body temperature or weight.

Figure 4.2 Taxol on days 6, 8, and 12 is ineffective at altering EAE.

Figure 4.3 EAE spinal cord lesions.

Figure 4.4 Lymphocyte re-stimulations. 
Figure 4.5 A single Taxol treatment has no effect on proliferation.

Figure 4.6 Proliferation occurs in intestines after single injection of Taxol

Figure 4.7 Schematic diagram depicting the effect of P-gp efflux pump expression on the paclitaxel concentrations surrounding cells over time in vitro and in vivo.

Table 4.1 Lymphocyte populations after Taxol treatments.

\section{Chapter 5}

Figure 5.1 Effect of Taxol on mice with EAE.

Figure 5.2 Taxol at $20 \mathrm{mg} / \mathrm{kg}$ delays the onset of EAE.

Figure 5.3 Weight-loss from Taxol-treatment.

Figure 5.4 Peloruside at $10 \mathrm{mg} / \mathrm{kg}$ delays the onset of EAE.

Figure 5.5 Effects of $20 \mathrm{mg} / \mathrm{kg}$ Taxol administered after disease onset.

Figure 5.6 Overlay of Taxol, peloruside, and control treatment results.

Figure 5.7 Toxicity of Taxol corresponds to initial onset of EAE symptoms.

Figure 5.8 Spinal cords from drug-treated mice with active EAE.

Figure 5.9 Populations of T cells from LN isolated from drug-treated mice with EAE.

Figure 5.10 Re-stimulation of $\mathrm{LN}$ cells that were isolated 30 days p.i.

Figure 5.11 Taxol does not affect MOG-specific T cell proliferation.

Table 5.1 Treatments of $20 \mathrm{mg} / \mathrm{kg}$ Taxol and $10 \mathrm{mg} / \mathrm{kg}$ peloruside significantly delay the onset of EAE.

Table 5.2 Comparisons between different drug treatments. 


\section{List of Abbreviations}

\begin{tabular}{|c|c|}
\hline AEC & - animal ethics committee \\
\hline APC & - antigen presenting cell \\
\hline $\mathbf{B B B}$ & - blood-brain barrier \\
\hline ВММФ & - bone marrow-derived macrophage \\
\hline $\mathbf{B R}$ & - brain \\
\hline Brd U & - 5-bromo-2-deoxyuridine \\
\hline BSA & - bovine serum albumin \\
\hline dPBS & - Dulbecco's cation-free PBS \\
\hline CD & - cluster of differentiation \\
\hline CCL & - chemokines ligands \\
\hline CFA & - complete Freund's adjuvant \\
\hline CFSE & - carboxyfluorescein succinimidyl ester \\
\hline CNS & - central nervous system \\
\hline Con A & - concanavalin A \\
\hline COX-2 & - cyclooxygenase-2 \\
\hline CTCM & - complete $\mathrm{T}$ cell media \\
\hline CTLA 4 & - cytotoxic T-lymphocyte-associated protein 4 \\
\hline $\mathrm{Cy}-\mathrm{C}$ & - CyChrome \\
\hline DC & - dendritic cell \\
\hline DLN & - draining lymph node \\
\hline DMEM & - Dulbecco's modified Eagles medium \\
\hline DMSO & - dimethyl sulfoxide \\
\hline EAE & - experimental autoimmune encephalomyelitis \\
\hline
\end{tabular}




\begin{tabular}{|c|c|}
\hline eIF4A & - eukaryotic initiation factor $4 \mathrm{~A}$ \\
\hline ELISA & - enzyme-linked immunosorbant assay \\
\hline FACS & - fluorescence activated cell sorting \\
\hline FCS & - foetal calf serum \\
\hline FITC & - fluorescein isothiocyanate \\
\hline GM-CSF & - granulocyte/macrophage colony stimulating factor \\
\hline H\&E & - hematoxylin and eosin \\
\hline HRP & - horseradish peroxidase \\
\hline ICAM 1 & - intracellular adhesion molecule 1 \\
\hline IFA & - incomplete Freund's adjuvant \\
\hline IFN- $\alpha$ & - interferon $\varepsilon$ \\
\hline IHC & - immunohistochemistry \\
\hline IL & - interleukin \\
\hline iNOS & - inducible nitric-oxide synthase \\
\hline INT & - intestine \\
\hline i.p. & - intraperitoneal \\
\hline i.v. & - intravenous \\
\hline $\mathbf{L N}$ & - lymph node tissue \\
\hline MBP & - myelin basic protein \\
\hline MLN & - mesenteric lymph node \\
\hline LPS & - lipopolysaccharide \\
\hline MAF & - Ministry of Agriculture and Forestry \\
\hline MHC & - major histocompatability complex \\
\hline MMP & - matrix metalloproteinases \\
\hline Мø & - macrophage \\
\hline
\end{tabular}




\begin{tabular}{|c|c|}
\hline MOG & - myelin oligodendrocyte glycoprotein \\
\hline MS & - multiple sclerosis \\
\hline M.tb. & - Mycobacterium tuberculosis \\
\hline MTD & - maximum tolerated dose \\
\hline MTT & -3-(4,5-dimethylthiazol-2-yl)-2,5-diphenyltetrazolium bromide \\
\hline NMR & - nuclear magnetic resonance \\
\hline NO & - nitric oxide \\
\hline PBS & - phosphate-buffered saline \\
\hline PEC & - peritoneal exudate cells \\
\hline Per-CP & - peridinin chlorophyll A protein \\
\hline p.i. & - post-immunisation \\
\hline PI & - propidium iodide \\
\hline PLP & - proteolipoprotein \\
\hline PTx & - pertussis toxin \\
\hline SA & - streptavidin \\
\hline SA-HRP & - streptavidin-horseradish peroxidase conjugate \\
\hline s.c. & - subcutaneous \\
\hline SCD & - spinal cord \\
\hline SEM & - standard error of the mean \\
\hline SH2D2A & $-\mathrm{SH} 2$ domain protein $2 \mathrm{~A}$ \\
\hline SPL & - splenic tissue \\
\hline TCR & $-\mathrm{T}$ cell receptor \\
\hline Th & $-\mathrm{T}$ helper cell \\
\hline TIRF & - total internal reflective fluorescence \\
\hline TLRs & - toll-like receptors \\
\hline
\end{tabular}




\section{TNF- $\alpha-$ tumor necrosis factor $\varepsilon$}

TPBS $\quad-0.05 \%$ Tween-20 in phosphate-buffered saline

VLA-4 - very late antigen 4 


\section{Chapter 1: Introduction}

\subsection{General Introduction}

Our knowledge of molecular biology and human diseases has expanded exponentially over the past century with exciting new discoveries announced time and time again. With the advent of new molecular techniques to identify novel drug targets, biotechnology is ever increasingly delving into innovative ways to treat difficult diseases. One such complex set of diseases includes those that fall under the category of autoimmunity. Because autoimmune disorders, such as systematic lupus erythematosus, rheumatoid arthritis, and multiple sclerosis, have no single known cause of disease, there is little that can be done to prevent the initial induction with the current technology. Treating autoimmunity after the onset of symptoms is proving just as difficult. Although the cellular mechanisms of disease are being elucidated, there is still no cure for autoimmunity. This situation, however, is bound to change. New targets are coming to light, while old targets are being revisited with novel approaches. The exciting field of biotechnology is in its infancy, and with all of the technical knowledge of today, we are barely scratching the surface of what we will see in the years to come.

This thesis is a condensed summarisation of a three-year project encompassing multiple aspects of autoimmunity and the treatment thereof. The findings presented herein may shed light on novel therapies for autoimmune diseases in general; however, the primary focus of this research revolves around multiple sclerosis. It is reasonable to say that, because of the multi-faceted nature of the immune system, a cure for multiple sclerosis may come at a cost. For those that suffer with 
autoimmunity, this cost may be well worth the risks. It is the hope of this researcher that this thesis can aid in the development of a therapy or cure for autoimmune disorders that has minimal impact on other systems aside from the intended target. To understand the therapy, one must understand the disease. 


\section{$1.2 \quad$ Multiple Sclerosis}

Multiple sclerosis (MS) is a disease caused by demyelination of nerves in the central nervous system (CNS), often leaving affected individuals in a debilitated state.

Although the cause is still a mystery, it is believed to be an inflammatory autoimmune disorder involving the destruction of myelin tissue by autoreactive immune cells in the CNS. Myelin sheaths surround nerve fibers, protecting and enhancing electrical impulses as they travel through the CNS. In MS, autoreactive immune cells are believed to target myelin, causing lesions that can interfere with nerve signal transduction in the CNS. The resulting inflammatory lesions can cause irreversible damage to the myelin-producing oligodendrocytes. Despite recent advances in our understanding of the disease, there are very few treatment options. To date, there is no cure.

\section{Cause of MS}

Over the years, epidemiological and population studies have attempted to tease out a specific cause of MS. Although there is still no single genetic or environmental factor responsible for this disorder, there are associated genetic and environmental risks with varying degrees of concordance that control the susceptibility to developing MS. It is estimated that MS affects 2.5 million people worldwide, women being 2 to 3 times more likely to develop MS than men. The predominance of MS in women, as well as the temporary improvement of disease during pregnancy, provides strong evidence of a hormonal link (Confavreux et al., 1998; Marrie, 2004). Several studies have identified a sharp rise in the total number of regulatory $\mathrm{T}$ cells $\left(\mathrm{T}_{\text {reg }}\right)$ during pregnancy (Somerset et al., 2004; Polanczyk et al., 2005), which are attributed to recovery from inflammation. The suppression of relapses and increased 
$\mathrm{T}_{\text {reg }}$ numbers during pregnancy may be attributed to the effects of high estradiol and/or low progesterone (Sicotte et al., 2002).

Hormonal differences alone cannot explain the occurrence of MS since there is also a clear genetic component, as shown by twin and family studies. If one identical twin develops MS, the likelihood of the second twin getting MS increases compared to non-identical twins (McFarland, 1992). Immediate family members of MS patients are also at an elevated risk for MS (Broadley et al., 2000; McFarland, 1992). The exact genes responsible for the increased risk of MS are still being investigated; however, a recent study reveals an association of MS incidence with variations in HLA class I and II alleles, TCRE, CTLA4, ICAM1, and SH2D2A, further supporting underlying genetic involvement (Dyment et al., 2004).

Although genetics has a role in MS, some people with many of the genetic risks do not develop the disease. A large body of evidence exists that points to environmental factors regulating disease induction. Because MS incidence increases the further one lives from the equator, it has been postulated that decreased levels of UV light in the wintertime, and the resulting decrease in vitamin D biosynthesis, is linked to MS induction (Ponsonby et al., 2002). Vitamin D has many immunological functions which supports the idea that deficiencies in vitamin D may increase the risk of developing MS (Cantorna, 2006; VanAmerongen et al., 2004). One study found that women who supplemented their diet with vitamin D had a significantly decreased chance of developing MS (Munger et al., 2004); however, other evidence suggests that vitamin D has very little effect in MS patients already afflicted with MS 
(Wingerchuk et al., 2005). Although vitamin D is frequently studied when pursuing an environmental cause of MS, it is only one aspect of this complex disease.

Simple changes in protein structures, including amino acid modifications or glycosylation, may initiate an autoimmune response. As an example, posttranslational modifications of myelin proteins have been implicated in MS (Beniac et al., 2000; Mamula et al., 1999; Raijmakers et al., 2005; van Boekel and van Venrooij, 2003). One of the more popular theories is that there is a viral or bacterial "trigger" that sets off a cascade of events, due to either molecular mimicry or bystander activation, that ultimately leads to myelin-reactive immune cells entering the CNS (Sospedra and Martin, 2005). An example of a post-infection neurological disorder is Guillan-Barre syndrome - caused by molecular mimicry - whereby an autoimmune response is initiated towards gangliosides because of their antigenic similarities to antigens from a primary infection. There is a strong concordance rate between the development of MS and previous infection with Epstein-Barr virus, human herpes-virus 6, chlamidia pneumoniae, or other infectious agents (Martyn et al., 1993; Moore et al., 2002; Sriram et al., 1998; respectively). In fact, one study found that anti-Epstein-Barr virus antibodies were found in 100\% of MS patients ( $\mathrm{n}$ = 107) (Wandinger et al., 2000). Taken together, these theories support the notion that MS is a multi-faceted disease requiring several inputs from both genetic and environmental sources in order for the onset to occur.

\section{Symptoms of MS}

The presentation of MS varies from case to case, but the most commonly experienced symptoms include numbness and tingling in the hands and feet, limb 
weakness, and optic neuritis. As the disease progresses, the symptoms may worsen to include loss of manual dexterity, deterioration in mobility, impaired thinking, muscle spasms, difficulty with speech and swallowing, and paralysis of extremities. Because many of these symptoms are not specific to MS, the diagnosis of MS is difficult and complex. There is no definitive test to confirm the diagnosis of MS; however, useful information comes from patient history, MRI scans, spinal taps to determine IgG levels, and serum antibody tests for myelin component reactivity.

There are four typical subsets of MS that are prevalent among patients: a) relapsingremitting, b) primary-progressive, c) secondary-progressive, and d) progressiverelapsing (Fig. 1.1). The most common form of MS at the time of original diagnosis is relapsing-remitting (Fig. 1.1 a), accounting for as many as $85 \%$ of all MS cases at the time of original diagnosis. It is characterised by distinct periods of CNS inflammation and worsening symptoms, followed by partial or complete recovery. Currently, there is no method to predict a relapse. Patients can be free from exacerbation for months or years, and then suddenly experience a relapse lasting for days or months. Relapsing-remitting MS frequently evolves into one of the other forms with age.

Patients suffering from secondary-progressive MS (Fig. $1.1 \mathrm{c}$ ) typically show symptoms similar to those with the relapsing-remitting form; however, the symptoms of secondary-progressive patients tend to steadily worsen with or without disease exacerbations. Once the disease becomes more progressive, minor recovery may or may not occur. It is estimated that approximately $50 \%$ of people who ultimately 
develop relapsing-remitting MS develop this form of disease within the first decade of initial diagnosis.

One of the less common MS subtypes, primary-progressive (Fig. 1.1 b), is characterised by a steady worsening of symptoms without defined periods of relapse. Plateaus and temporary improvements are not uncommon, and the rate of degeneration may vary. This form of MS generally affects less than 10\% of patients.

Even less common, at $5 \%$ of MS patients, is the progressive-relapsing form of disease (Fig. 1.1 d). From the onset, these patients exhibit progressive symptoms with clearly defined inflammatory relapses. Minor recovery may follow these flareups; but, unlike relapsing-remitting MS, there is a continuous progression of disease severity. A more comprehensive collection of MS information can be found on the website for the National MS Society (www.nationalmssociety.org) in the USA. 


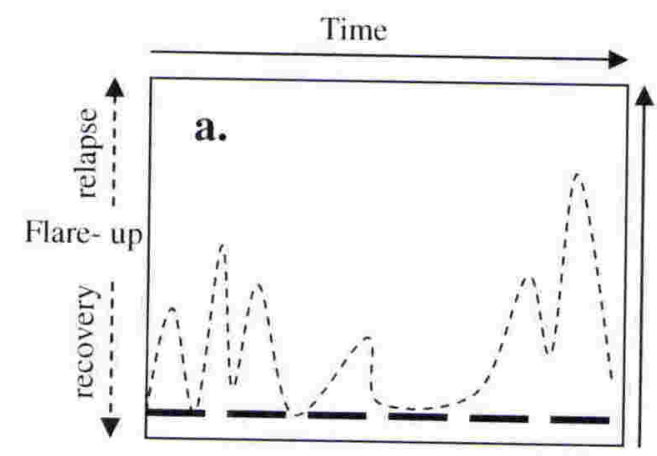

Relapsing-Remitting

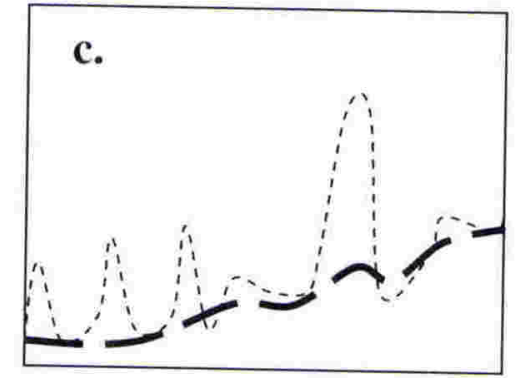

Secondary-Progressive

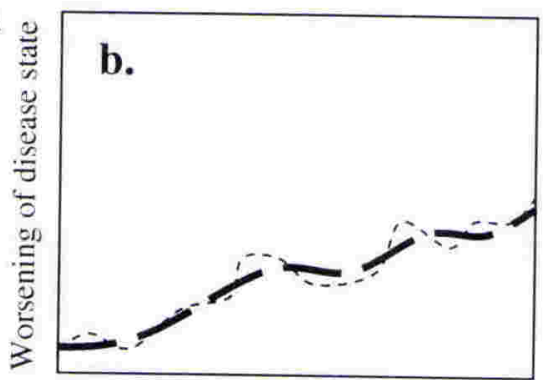

Primary-Progressive

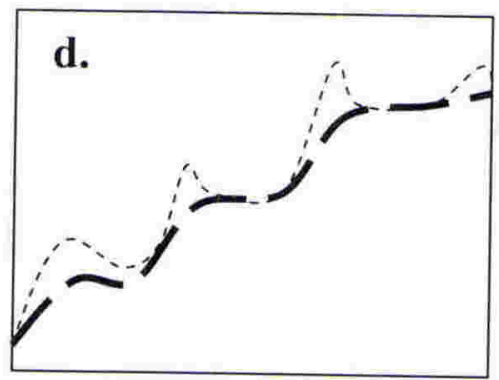

Progressive-Relapsing

- $=$ Overall level of disease

$=$ Relapse/recovery of exacerbations

\section{Figure 1.1 The four subtypes of MS}

The common manifestations of MS - relapsing-remitting (a), primary-progressive (b), secondary-progressive (c), and progressive-relapsing (d) - are shown above. Relapses are represented by short dashes (---) and overall paralysis is shown by long dashes $(---)$. 


\section{$\underline{\text { Etiology of MS }}$}

Interestingly, the etiology of these different MS subtypes does not correlate with a specific cellular cause. The lesions themselves show a considerable amount of heterogeneity, with underlying CNS pathology ranging from oligodendropathy and little inflammation to highly inflamed lesions dominated by $\mathrm{CD} 8^{+} \mathrm{T}$ cells and macrophages (Lucchinetti et al., 2000). Antibody involvement has also been attributed to the formation of CNS lesions (Walsh et al., 1986; Baranzini et al., 1999), and the highly elevated presence of antibodies in the cerebral spinal fluid is common in MS patients (Kabat et al., 1950), which contributes to the diagnosis of MS. Despite the variation of lesion presentation in later stages of disease, MS is still generally considered a CD4 ${ }^{+} \mathrm{Th}_{1}$-mediated disease, especially in the earlier stages (Hellings et al., 2002; Sospedra and Martin, 2005; Stinissen et al., 1997). This belief is supported by the fact that $\mathrm{CD} 4^{+} \mathrm{T}$ cells are, at least in part, necessary for the B cell and $\mathrm{CD}^{+} \mathrm{T}$ cell maturation. Moreover, the function of two of the most promising genetic susceptibility candidates, class II-restricted HLA-DR and-DQ, is primarily associated with $\mathrm{CD} 4^{+} \mathrm{T}$ cells. But, not all $\mathrm{CD} 4^{+} \mathrm{T}$ cells are the same. It has been shown that both MS patients and normal, healthy people have $\mathrm{CD} 4^{+} \mathrm{T}$ cells that respond to myelin proteins (Burns et al., 1983), so auto-reactive T cells alone cannot explain why some individuals get MS and others don't. Interestingly, MS patients generally have a greater abundance of autoreactive T cells with $1 / 10^{4}$ or more T cells being myelin-reactive (Muraro et al., 2003; Hong et al., 2004) indicating clonal T cell expansion. Teasing out the overall picture of MS development gets complicated considering difficulties of obtaining pathological information from MS patients in a non-invasive way. 
Although a common and specific initiation event for MS has not been identified and there is a variation of the general presentation of disease, as well as the heterogeneity of lesions, there is enough evidence to piece together the pathophysiological similarities that lead to the development of MS. As evidenced above, the early stages of MS are primarily dominated by myelin reactive $\mathrm{CD} 4^{+} \mathrm{T}$ cells of the $\mathrm{Th}_{1}$ variety. In conjunction with antigen presenting cells (APC) displaying HLA-DR or -DQ, CD4 ${ }^{+}$ $\mathrm{T}$ cells become activated in response to myelin proteins. These cells then expand, but individuals may have variation in the downstream activation of other immune cell activity, including mast cells, $\mathrm{CD} 8^{+} \mathrm{T}$ cells, and $\mathrm{B}$ cells, which may explain the phenotypic deviation and the presence of $\mathrm{Th}_{2}$-type cells in what is considered $a \mathrm{Th}_{1}$ mediated disease. This basic model explains the initial events and inflammatory relapses, but most MS patients present with relapsing and remitting phases.

A naturally suppressive subset of $\mathrm{CD} 4^{+} \mathrm{T}$ cells exists that are also $\mathrm{CD} 25^{+}$and express FoxP3. These regulatory $\mathrm{T}$ cells $\left(\mathrm{T}_{\text {reg }}\right)$ are involved in the resolution phase of immune responses. In MS patients, however, overall $\mathrm{T}_{\text {reg }}$ numbers are generally present at lower levels and the $\mathrm{T}_{\text {reg }}$ functions are not as suppressive (Viglietta et al., 2004), suggesting that there might be an inability to effectively suppress a flare-up, or an impaired resolution of inflammation. Studies on $\mathrm{T}_{\text {reg }}$ involvement of autoimmunity are in the early stages, but the evidence presented thus far gives a strong indication that $\mathrm{T}_{\text {regs }}$ may hold the key to ameliorating inflammation.

Nonetheless, $\mathrm{CD} 4^{+} \mathrm{T}$ cells as a whole present an opportunity to develop drugs that halt the initiation events of an inflammatory relapse, thus leading to an improvement of MS symptoms. The $\mathrm{CD}^{+} \mathrm{T}$ cell hypothesis is further supported by studies done in 
the experimental autoimmune encephalomyelitis (EAE) animal model of MS (Sospedra and Martin, 2005). 


\subsection{Experimental Autoimmune Encephalomyelitis}

There are written accounts and drawings from as far back as the Middle Ages describing clearly what we know now to be MS; however, the recognition of MS as a specific disease - i.e., not "madness" - did not occur until the later decades of the $19^{\text {th }}$ century. The understanding of this disease grew at a very slow pace during the first few decades of the $20^{\text {th }}$ century. During this time, MS was believed to be virusinduced. In 1935, Dr. Thomas Rivers of the Rockefeller Institute in New York City injected virus-free myelin into laboratory animals, ultimately causing an autoimmune reaction to myelin (Rivers and Schwentker, 1935). The subsequent disease, now referred to as EAE, had many of the characteristics of MS. The development of the EAE animal model marked a turning point for research into MS. To date, the EAE animal model has provided valuable insight into MS pathophysiology, and it is still the gold standard disease prototype. It is well known, however, that MS is not as simple or straight forward as the EAE model (Sriram and Steiner, 2005; Steinman and Zamvil, 2005), but the similarities have helped MS researchers around the world develop an understanding of the human disease, as well as inflammatory autoimmune diseases in general.

The EAE model has improved markedly since the days of Dr. Rivers' initial experiments. The model Dr. Rivers employed required 85 injections of rabbit brain extracts over the course of a year to induce EAE in primates (Rivers and Schwentker, 1935). Today, with the help of complete Freund's adjuvant (CFA), the number of injections of brain extract to bring about disease has been reduced to one. In addition, variations in the EAE phenotype can be induced using different animals or different 
myelin peptide fragments. Although each model has its own advantages and disadvantages, they all have similarities to MS and its various sub-types.

Although the primate EAE model still exists, it is relatively inconvenient to do studies considering the availability and ease of murine models of disease. In fact, the murine models of EAE may be more suitable for the application of treatments to human MS because of the similarities each bears to the various sub-types of MS. For example, SJL mice with proteolipoprotein-induced EAE phenotypically display several relapsing-remitting episodes of disease. One proposed mechanism for relapsing EAE is believed to be epitope spreading (Miller et al., 2007), which has also been postulated as a trigger for flare-ups in human MS (Sospedra et al., 2005). Another example of EAE and MS similarities is the Lewis rat model when immunised with spinal cord homogenate, which generally results in a single bout of CNS inflammation followed by a partial or complete remission. Parallels have been drawn between the Lewis rat model of EAE and primary-progressive MS. Some of the newer animal models included in the EAE family involve transgenic mice, including some that express $\mathrm{T}$ cells specific to myelin antigens. Specifically, the 2D2 transgenic EAE mouse model expresses $\mathrm{CD} 4^{+} \mathrm{T}$ cells with a specificity towards MOG $_{35-55}$ peptide (Bettelli et al., 2003). Strikingly similar to MS, the resulting CNS inflammation may be spontaneous and is generally preceded by optic neuritis. Various other EAE models have been utilised for their MS-like properties (Skundric, 2005), and more models are being developed that better represent the most current understanding of MS etiology. 
When left non-immunised, most experimental animal strains do not typically develop EAE. Disease can, however, be actively induced in normal healthy animals. The EAE animal model is a result of initiating a $\mathrm{CD} 4^{+} \mathrm{T}$ cell response towards a specific myelin antigen. Myelin antigens commonly used for this purpose are myelin basic protein (MBP), proteolipoprotein ( $\mathrm{PLP}_{139-151}$ ), and myelin oligodendrocyte glycoprotein $\left(\mathrm{MOG}_{35-55}\right)$. These antigens, in addition to complete Freund's adjuvant (CFA), comprise the immunisation that causes actively induced EAE. EAE can also be passively induced by transferring encephalitogenic $\mathrm{T}$ cells isolated from an animal with EAE into a normal healthy animal of the same haplotype. Passive induction can also be termed "adoptive transfer" and because this method by-passes the activation/priming stage when naïve $\mathrm{T}$ cells are activated, it is used to focus on the later stages of the disease process involving CNS infiltration and nerve tissue damage. The experiments described in this thesis will solely use active induction of EAE in mice.

The EAE animal model used for the studies in this thesis is the C57BL/6 mouse. This strain is commonly used, and, when EAE is induced, it is characterised by typical disease onset two weeks post-immunisation (p.i.) followed by a partial remission by 4 weeks p.i. There are many advantages to using this model, including high disease incidence (80\%-100\%) and a consistent and progressive pattern of paralysis. Both early-stage MS and the C57BL/6 model of EAE are considered $\mathrm{CD}^{+} \mathrm{Th}_{1}$-mediated diseases, thus providing a strong correlation between the work undertaken herein and the translation of these results into possible outcomes for the treatment of MS. 


\subsection{EAE Pathophysiology}

Negative selection in the thymus during $\mathrm{T}$ cell development is believed to eliminate the vast majority of autoreactive T cells; however, there are autoreactive $\mathrm{T}$ cell "escapees" that have the potential to induce autoimmunity (Wekerle and Linington, 2006). The activities of these rogue $\mathrm{T}$ cells are generally kept under control during normal immune function, and it takes the combination of auto-antigen with a strong inflammatory adjuvant to activate the naïve autoreactive T cells. Even after activation of the naïve cells, the cells must be able to find their target organ/tissues to cause disease.

The C57BL/6 model of actively induced EAE is brought about by immunising each mouse subcutaneously with $50 \mathrm{\varepsilon g}$ of $\mathrm{MOG}_{35-55}$ peptide emulsified in complete Freund's adjuvant (CFA); CFA contains incomplete Freund's adjuvant (IFA) and heat-killed Mycobacterium tuberculosis (M.tb.). Although MOG peptide is quantitatively a minor component of myelin, it is still able to induce a powerful antigenic response in MOG-reactive T cells. In conjunction with the strong toll-like receptor (TLR) agonist, M.tb., antigen-presenting cells (APCs) presenting MOGloaded MHC II molecules will activate the $\mathrm{CD}^{+} \mathrm{T}$ cells expressing a MOG-specific T cell receptor (TCR). Along with myelin peptide, other toll-like receptor agonists emulsified in IFA, such as peptidoglycan (Visser et al., 2005) or CpG oligonucleotides (Segal et al., 2000), have successfully induced EAE, further emphasising the necessity of a TLR agonist for disease induction.

To enhance the encephalogenic response, the addition of intraperitoneally injected pertussis toxin (PTx) is required on the day of immunisation, as well as 2 days post- 
immunisation (Lee and Olitsky, 1955). It has been suggested that PTx facilitates permeability of the blood-brain barrier (BBB), thereby allowing access of encephalogenic T cells to the brain and spinal cord (Kerfoot et al., 2004; Yong et al., 1993). Other research shows that PTx augments the APC response in lymphoid tissue and in the CNS, enhancing T cell activation and clonal expansion (Hofstetter et al., 2002). Regardless of its mechanism of action, PTx clearly augments the diseaseinducing immunisation, resulting in an increased incidence of disease.

The EAE priming process is meant to activate, differentiate, and expand naïve T cells with a TCR-specificity toward $\mathrm{MOG}_{35-55}$ peptide into encephalogenic $\mathrm{Th}_{1}$ cells. The mechanism behind this specific $\mathrm{T}$ cell activation and $\mathrm{Th}_{1}$ differentiation lies in the priming of APCs, such as dendritic cells (DC) and macrophages (MØ), with bacterial products within the CFA (as previously mentioned) that stimulate TLRs. At the site of immunisation, the primary APC responsible for antigen presentation is the DC. The immunisation ligates specific TLRs on DCs, causing them to mature. Mature APCs produce large amounts of inflammatory cytokines and mediators including IL1, IL-12, and TNF- $\varepsilon$. The mature APCs then migrate to the draining lymph nodes (DLN) and present MOG antigen on MHC II to CD4 $4^{+}$T cells (Targoni et al., 2001). The inflammatory environment introduced by the APCs induces the $\mathrm{CD}^{+}$MOGreactive $\mathrm{T}$ cells to respond in a manner considered to be representative of the $\mathrm{Th}_{1}$, or inflammatory, variety. Once $\mathrm{Th}_{1} \mathrm{CD} 4^{+} \mathrm{T}$ cells are activated, they proliferate rapidly and secrete lymphotoxin, IFN-E, and TNF- $\varepsilon$ (Juedes et al., 2000). These cytokines further stimulate APCs, causing a positive feedback cycle of inflammation. The activated MOG-reactive T cells circulate throughout the body, constantly scanning APCs for MOG peptides presented on MHC II molecules. The numbers of MOG- 
reactive $\mathrm{T}$ cells peak in the periphery shortly before the mice display symptoms of disease (Targoni et al., 2001), which occurs approximately 7-10 days after immunisation in C57BL/6 mice.

Because MOG is only found in the CNS, an immunoprivileged site, the MOGreactive $T$ cells must cross the $\mathrm{BBB}$ to gain access to the antigen-containing tissue. As mentioned earlier, PTx may aid in facilitating the movement of these cells across the BBB; however, a T cell can enter the CNS without PTx, but only when activated, and entry is independent of its TCR specificity (Hickey, 1999). These activated MOG-reactive T cells migrate into the CNS between day 7 post-immunisation (Juedes et al., 2000) and the day prior to disease onset (Targoni et al., 2001). Once across the BBB, the T cells recognise MOG presented by various APCs, both exogenous (MØ) and endogenous (microglia [MG]). Interestingly, these CNSinfiltrating $\mathrm{T}$ cells show very little proliferation, if any, in response to myelin presented on APC's within the CNS (Ohmori et al., 1992). Upon TCR stimulation within the BBB, the activated MOG-reactive T cells respond by secreting inflammatory cytokines (as mentioned above) and chemokines such as CCL3 and CCL4 (Chavarria and Alcocer-Varela, 2004; Karpus and Ransohoff, 1998). In addition, resident APCs secrete other chemokines, such as CCL2 and CCL5, that serve to further enhance T cell activities (Chavarria and Alcocer-Varela, 2004; Juedes et al., 2000). The chemotaxic cytokine trails draw M $\varnothing$, mast cells, B cells, and DCs into the CNS. The constant supply of inflammatory cytokines delivered back and forth between T cells and APCs leads to repetitive stimulation and massive destruction of the myelin and myelin-producing oligodendrocytes (Ubogu et al., 
2005), ultimately causing lesions in the perivascular regions of the brain and spinal cord (Fig. 1.2).

The inflammation cycle does not proceed unchecked, since C57BL/6 mice with EAE do, in fact, recover from disease, albeit only partially. The cause of recovery is primarily associated with $\mathrm{CD} 4^{+} \mathrm{CD} 25^{+} \mathrm{FoxP}^{+} \mathrm{T}_{\text {reg }}$ cells. When $\mathrm{T}_{\text {reg }}$ cells are depleted from mice during EAE, natural recovery is inhibited (McGeachy et al., 2005). Moreover, low numbers of $\mathrm{CD}^{+} \mathrm{CD}^{+} 5^{+}$cells isolated from the CNS of recovering mice confers protection from EAE development when these cells are passively transferred to immunised mice (McGeachy et al., 2005). On activation, these cells produce potent anti-inflammatory cytokines, including TGF- $\varepsilon$ and IL-10, which are believed to be responsible for downregulating inflammatory interactions between APCs and autoreactive T cells in the CNS (Mekala et al., 2005; Zhang et al., 2004). $\mathrm{T}_{\text {reg }}$ cells are currently a topic of intensive investigation, both in EAE and MS, and multiple subsets of $\mathrm{T}_{\text {reg }}$ cells have been identified with varying roles in different diseases.

From immunisation of autoantigens to recovery from disease, EAE exhibits a complex pattern of CNS demyelination comparable to human MS. Sospedra and Martin (2005) have recently reviewed the factors involved in demyelination during MS development, and a summary diagram from their paper is reproduced in Fig. 1.3. The C57BL/6 EAE model provides a consistent disease phenotype by which many studies have attempted to tease out the complexities of autoimmune interactions. Current cellular/pathophysiological theories have been described above, but they are constantly changing or being improved upon as new studies continue to delineate and 
analyse additional aspects of the disease. For further information about the cellular pathologies of MS and EAE, please refer to the comprehensive review article by El Behi et al., 2005, which details the activities of the major immune cells in both EAE and MS. Ultimately, the $\mathrm{CD} 4^{+} \mathrm{T}$ cell response that is fundamental in the early stages of EAE and MS has been described many times before. It is this fundamental characteristic that provides a defined target for novel treatments that may directly translate into a clinical therapy for MS. 

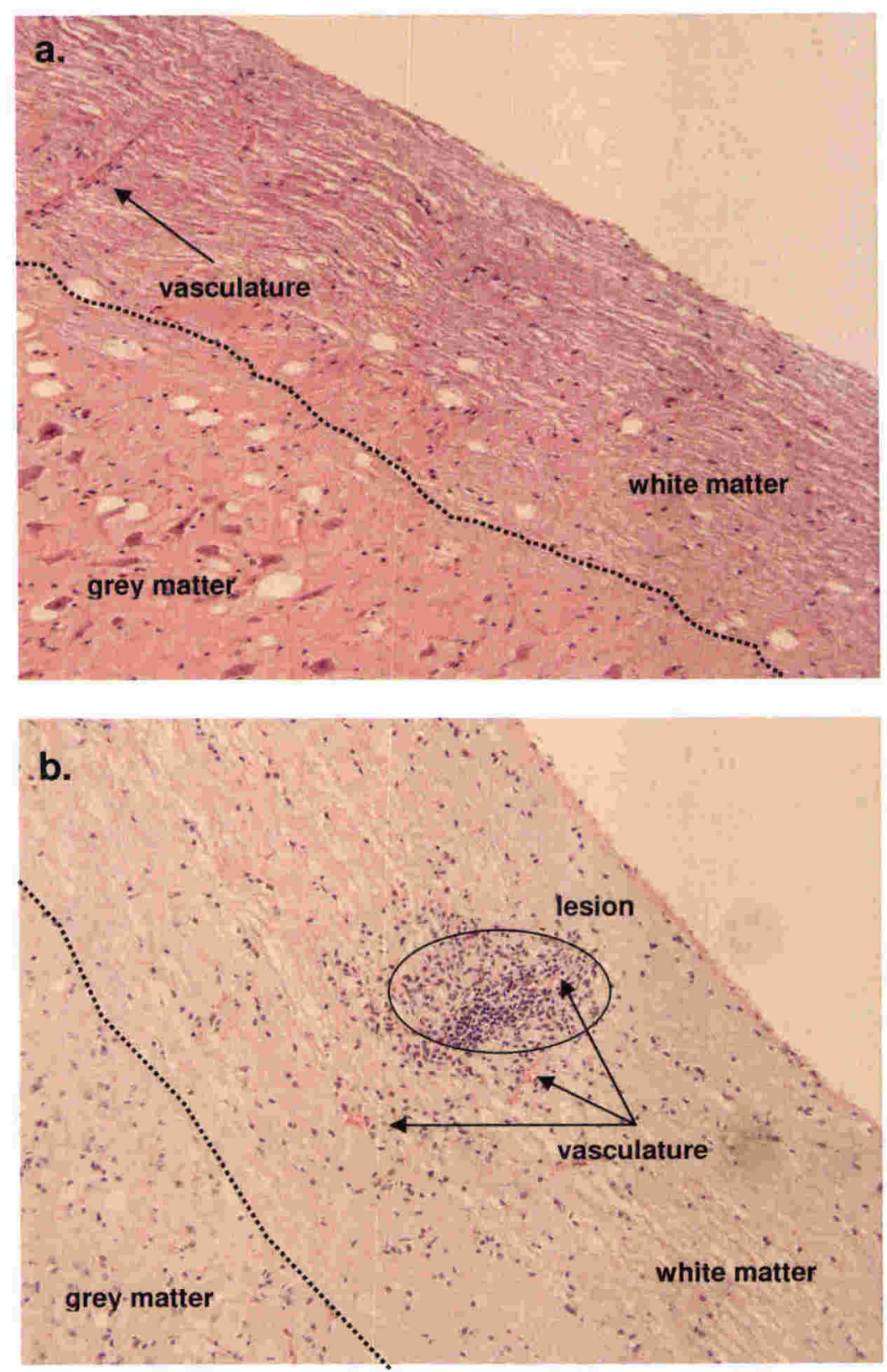

Figure 1.2 Hematoxylin and eosin (H\&E) stained longitudinal sections of spinal cords. Above are pictures of a normal spinal cord (a) and a spinal cord from a mouse with EAE (b). The white matter, which contains the myelinated nerve fibers, is discernible by the long neuronal tracts. Also labeled is the grey matter, and a dotted line is drawn between them for ease of identification. A typical lesion is circled in Panel b, and is characterised by the accumulation of nucleated cellular infiltrates. The arrows point to three small sections of red blood cells in the vasculature (darker pink appearance). Autoimmune cell infiltration across the BBB initiates from within the brain vasculature. These pictures were taken with a $100 x$ magnification. 


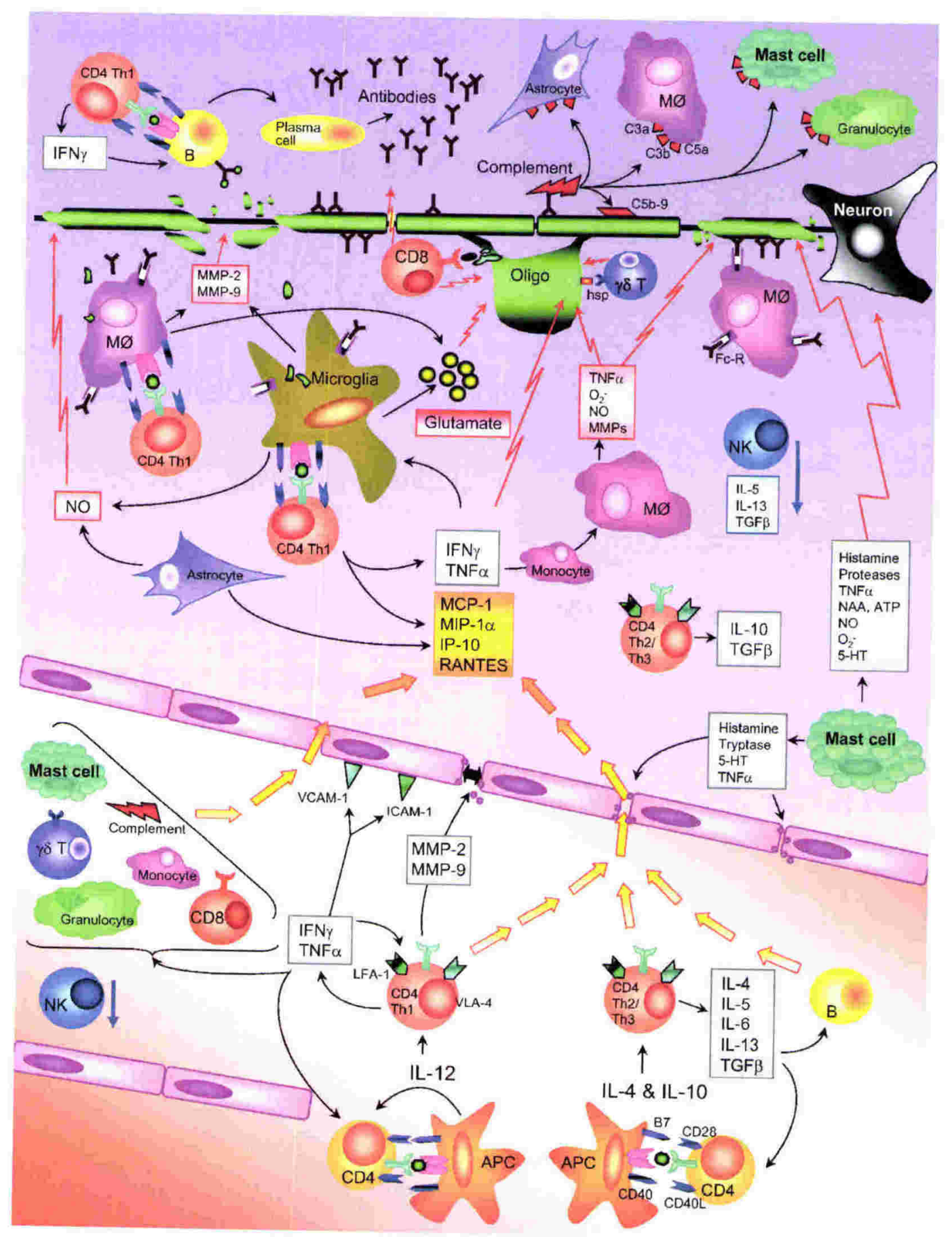

(Sospedra and Martin, 2005, with permission from the journal)

Figure 1.3 A schematic interpretation of the molecular and pathological events that lead to myelin destruction in MS. 


\subsection{Current MS Treatments and Investigational Therapies}

The current knowledge of MS is limited by the ability of researchers to perform indepth cellular and physiological analyses in patients suffering from the disease.

Although clinical studies on MS have been carried out, many experiments would be too invasive and unethical to perform in humans, which is why the EAE animal model is so useful. The EAE animal model has been crucial for the development of current drugs and for evaluating potential therapies to treat MS (Steinman, 1999; Steinman and Zamvil, 2005). The downside, however, is that not all drugs that are effective in EAE will have therapeutic effects in MS (Steinman, 1999; Steinman and Zamvil, 2005). It is suggested that the rationale behind designing therapies for MS in the EAE model can be tricky due to the relatively short treatment durations in EAE and lack of multi-point screening of side-effects in animals (Steinman and Zamvil, 2005). For this reason, drug therapies should target an activity that is present in both MS and EAE.

\section{$\underline{\text { Glatiramer acetate }}$}

All of the current MS treatments have successfully ameliorated EAE by preventing or blocking a common mechanism found in both diseases. One of the more successful FDA-approved MS therapies to date is glatiramer acetate which shows a relapse reduction rate of $30 \%$ (Johnson et al., 1995). Glatiramer acetate consists of random-length synthetic polypeptides containing fixed molar ratios of four amino acids found in myelin: tyrosine, glutamate, alanine, and lysine. Originally used in an attempt to initiate EAE, it was found to have the opposite effect. Its mechanism of action is still unclear, but current studies indicate that glatiramer acetate binds tightly to MHC II on APCs (Yong, 2002). Instead of inducing the typical $\mathrm{Th}_{1}$ response 
found in MS/EAE, the resulting $\mathrm{T}$ helper cells take on a $\mathrm{Th}_{2}$ phenotype and secrete anti-inflammatory cytokines such as IL-4, IL-5, and IL-10. Further evidence suggests that this tight binding to MCH II by glatiramer acetate causes T cell anergy (Schmied et al., 2003), probably due to the prevention of antigen presentation to effector $\mathrm{T}$ cells. There are few side-effects associated with glatiramer acetate, and the safety profile is very clean; however, only $30 \%$ of MS patients show any benefit from this treatment.

\section{Interferon- $\varepsilon$}

Another FDA-approved treatment that has been used with success rates similar to those of glatiramer acetate is the cytokine, interferon- $\varepsilon$. With only a $30 \%$ relapse reduction rate, however, it, like glatiramer acetate, is falling well short of an ideal treatment (Feldmann and Steinman, 2005). Interferon- $\varepsilon$ works through an entirely different mechanism compared to glatiramer acetate. The therapeutic action exerted by interferon- $\varepsilon$ prevents upregulation of MHC II on APCs, while downregulating costimulatory molecules (Yong, 2002). In addition, interferon- $\varepsilon$ has anti-proliferative effects on T cell clones (Yong, 2002).

\section{$\underline{\text { Natalizumab }}$}

One of the strategies employed to treat MS is to target the adhesion molecule $\varepsilon_{4}$ integrin with monoclonal antibodies. The treatment, called natalizumab, prevents VLA-4 on autoreactive T cells from binding to $\varepsilon_{4}$-integrin, ultimately preventing $\mathrm{T}$ cell adhesion and migration across the BBB (von Andrian and Engelhardt, 2003). Although natalizumab is more successful than the other drugs detailed above, with a $66 \%$ reduction in annual MS relapse rates, it is closely associated with a rare, but 
potentially fatal disease known as progressive multifocal leukoencephalopathy (PML) (Bennett, 2006). The safety profile of natalizumab is currently being reviewed by the FDA in the United States of America. If this drug is approved for all MS patients, it would have the highest relapse-reduction rate of all clinically available treatments. One of its disadvantages, however, is that it must be administered regularly to maintain a therapeutic effect.

\section{Mitoxantrone and Pixantrone}

Another clinically approved treatment for MS is the anti-proliferative compound, mitoxantrone. Mitoxantrone is a topoisomerase II inhibitor that decreases overall numbers of B cells and T cells, as well as maintains T cell suppression (Fidler et al., 1986; Gbadamosi et al., 2003). Mitoxantrone successfully ameliorates EAE and has had success in MS (Gonsette, 1996; Ridge et al., 1985). However, there is an increasing concern that long-term use of mitoxantrone can cause cardiotoxicity (Avasarala et al., 2003). A related alternative drug with no known cardiotoxicity problems, pixantrone, is proving to be a possible successor to mitoxantrone due to its greater anti-proliferative effects (Cavaletti et al., 2004). Until pixantrone is approved by the FDA, mitoxantrone is the only anti-proliferative drug on the market for treatment of MS. All of the currently approved treatments for MS are summarised in Table 1.1. 


\begin{tabular}{|c|c|c|c|c|c|}
\hline Trade name & Generic name & Type of drug & Mechanism & \begin{tabular}{|l|} 
Approx. $\%$ \\
of relapse \\
reduction
\end{tabular} & \begin{tabular}{|l|} 
Year of \\
introduction \\
to the market
\end{tabular} \\
\hline Copaxone® & $\begin{array}{l}\text { glatiramer } \\
\text { acetate }\end{array}$ & $\begin{array}{l}\text { Random chain } \\
\text { polymer of } \\
\text { amino acids }\end{array}$ & Unknown & $30-35 \%$ & 1996 \\
\hline Avonex $\circledast$ & interferon- $B$ 1-a & Cytokine & Unknown & $30-35 \%$ & 1996 \\
\hline Rebif ${ }^{\circ}$ & interferon- $B$ 1-a & Cytokine & Unknown & $30-35 \%$ & 2002 \\
\hline Betaseron ${ }^{\circledR}$ & interferon- $B 1-b$ & Cytokine & Unknown & $30-35 \%$ & 1993 \\
\hline Tysabri® & natalizumab & $\begin{array}{l}\text { Mouse } \\
\text { antibody }\end{array}$ & $\begin{array}{l}\text { Prevents VLA-4 } \\
\text { from binding a4- } \\
\text { integrin }\end{array}$ & $60-70 \%$ & 2004 \\
\hline Novantrone $\AA$ & mitoxantrone & $\begin{array}{l}\text { Topoisomerase } \\
\text { II inhibitor }\end{array}$ & $\begin{array}{l}\text { Reduces overall } \\
T \text { and } B \text { cell } \\
\text { numbers }\end{array}$ & $30-35 \%$ & 2000 \\
\hline
\end{tabular}

\section{Table 1.1 Current MS therapies.}

The research into MS treatments cover various aspects of disease, including simple remedies such as dietary changes to include more vitamin D or oral tolerance of myelin antigens, but the treatments extend beyond this to involve the manipulation of the immune response in general. Other possible therapies might even come from parasites and associated antigens. Evidence suggests that Schistosoma mansoni antigens ameliorate EAE through a strong Th2-inducing mechanism via the STAT6 pathway (La Flamme et al., 2003; Sewell et al., 2003). There are dozens, if not hundreds, of treatments that have proven to have some ameliorative effect in EAE and are currently involved in clinical trials or awaiting approval for clinical trials. Ideally, the most successful drug will remove autoreactive immune cells and restore 
normal immune system activity. This has never been achieved, leaving ample room for further research into a cure for MS. 


\subsection{Anti-proliferative Agents and the Treatment of Hyper- proliferative Diseases}

As previously mentioned, mitoxantrone is an anti-proliferative agent that has been approved by the FDA to treat MS. It works by inhibiting the topoisomerase II enzyme, effectively preventing cell division in interphase by slowing cells in $\mathrm{S}$ phase of the cell cycle and halting cells in $\mathrm{G}_{2}$ phase (Feofanov et al., 1999). Topoisomerase II is one of many possible targets that are involved in cell-cycle control.

One particular group of anti-proliferative agents that bind microtubules or soluble tubulin prevents proliferation during mitosis, or $\mathrm{M}$ phase of the cell cycle, generally by disrupting microtubular action. The primary function of microtubules during anaphase of mitosis is to segregate duplicated chromosomes (Pellman, 2001). Because of this, microtubule disruption is a popular method to treat cancer. Chromosomal segregation, however, is not the only role of microtubules. During normal cell function, microtubules play a major part in scaffolding, migration, and intracellular transport (Schiff and Horwitz, 1980). Although there is a variety of known microtubule disrupting agents (Attard et al., 2006), most of these compounds are difficult to obtain or are very costly.

\section{$\underline{\text { Paclitaxel }}$}

Paclitaxel is a naturally occurring compound isolated from the Pacific yew tree (Wani et al., 1971). It is widely used in the clinical setting as a chemotherapeutic drug (Bhalla, 2003), and its relative abundance makes it inexpensive and accessible. The potent anti-tumor activity exhibited by paclitaxel is primarily due to its ability to 
arrest dividing cells in $\mathrm{G}_{2} / \mathrm{M}$ phase by preventing microtubule depolymerisation, ultimately causing arrested cells to apoptose (Bhalla, 2003; Donaldson et al., 1994; Fuchs and Johnson, 1978; Manfredi et al., 1982; Rowinsky et al., 1988). While the focus of paclitaxel research has been to target cancer, cancer cells are not the only types of cells affected by paclitaxel. For example, the proliferation of stimulated lymphocytes is inhibited after exposure to paclitaxel in cultures (Brown et al., 1985; Chuang et al., 1994). Patients treated with paclitaxel experience many side-effects, including a decrease in the number of lymphocytes, likely due to the anti-mitotic nature of the drug (Kotsakis et al., 2000; Sako et al., 2004; Tsavaris et al., 2002). Given these inhibitory effects on immune cell proliferation, paclitaxel may have the desired immunomodifying activities needed for treatment of EAE or MS, since CD4 ${ }^{+}$ T lymphocytes must undergo mitosis to clonally expand.

Paclitaxel has previously been used as an immunomodifier in autoimmunity. A micellar formulation of paclitaxel successfully delayed or prevented the onset of EAE in Lewis rats (Cao et al., 2000). It was theorised that the impact on EAE was directly related to the anti-mitotic nature of paclitaxel. The authors, however, did not investigate the mechanisms behind this delay of onset. Other studies found that paclitaxel could impact collagen-induced arthritis and prevent the onset of disease altogether (Brahn et al., 1994; Oliver et al., 1994). Yet again, the mechanisms of prevention were not investigated. Although collagen-induced arthritis is an entirely different disease from EAE, both diseases are believed to be primarily mediated by $\mathrm{CD}^{+} \mathrm{T}$ cells and can be induced by immunisation with a self antigen in CFA (Ercolini and Miller, 2006; Sakaguchi and Sakaguchi, 2005). 


\section{$\underline{\text { Peloruside A }}$}

The novel compound, peloruside A (peloruside), was discovered in extracts of Mycale hentsheli, a marine sponge that lives in the coastal regions of New Zealand (West et al., 2000). Like paclitaxel, peloruside stabilises microtubules and has potent anti-mitotic activity in cancer cell lines, causing proliferating cells to apoptose in the $\mathrm{G}_{2} / \mathrm{M}$ phase of the cell cycle (Hood et al., 2001; Hood et al., 2002). Although the supply is limited, peloruside is exclusively available for use in selected projects at Victoria University of Wellington. The paclitaxel-like properties of peloruside make it an interesting molecule to study for its therapeutic potential in cancer and other hyperproliferative disorders, including autoimmunity.
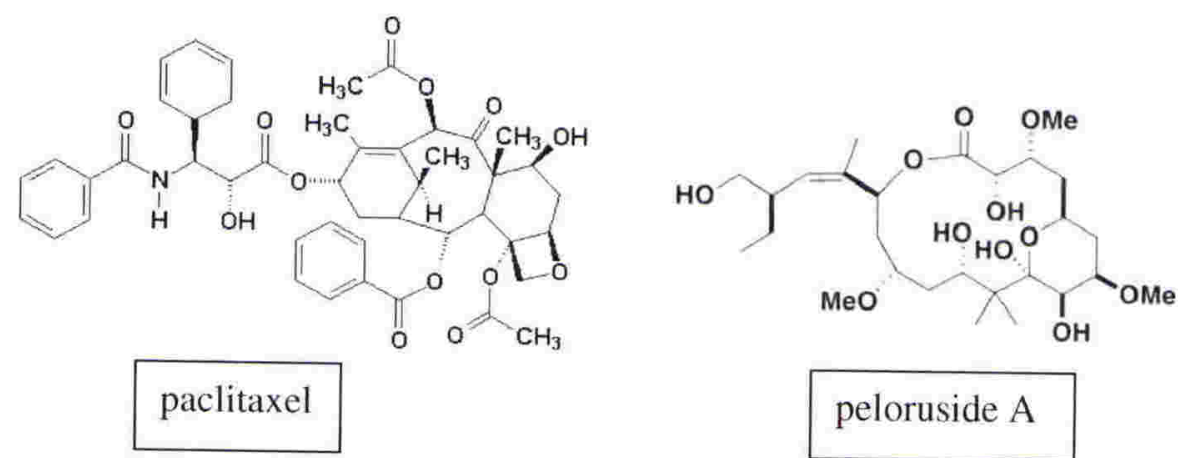

peloruside $\mathrm{A}$

Figure 1.4 Structures of the anti-mitotic, microtubule-stabilising compounds, paclitaxel and peloruside $\mathrm{A}$. 


\subsection{Aims}

Many studies over the years have attempted to dissect every aspect of MS. Due to limitations in the models used, many assumptions have been made in regard to the etiology of MS by reference to findings in the EAE animal model of MS. If one delves into the mechanisms of the two distinct, but similar, diseases, then differences will undoubtedly be found in their respective pathophysiologies. This is why many successful treatments of EAE do not directly translate into MS therapies. The variations in the cellular immunology of MS can vary between patients, similarly to the variations of different EAE animal models. There are, however, commonalities that are often overlooked when searching for the next treatment. It is the fundamental similarities of the two diseases that should be targeted in order to increase the likelihood of crossover therapeutic success.

The hallmark of any immune response is the rapid proliferation of cells responding to a foreign antigen. What separates inflammatory autoimmune responses from other immune events is the fact that they involve lymphocytes that target "self" antigens. During the initial phases of both EAE and MS, a large number of proliferating autoreactive $\mathrm{CD}^{+} \mathrm{Th}_{1}$ cells are present. In theory, very few immune cells are normally proliferating at any given time in healthy animals. When disease is initiated in EAE mice, the vast majority of proliferating immune cells should be responding to self-peptide or adjuvant; thus, targeting cell division in general may have a weighted effect on this cell population and open doors to therapeutic intervention in EAE and MS. 
It is the intention of this project to target the proliferating cell populations responsible for EAE - especially $\mathrm{CD}^{+}{ }^{+} \mathrm{T}$ cells - with anti-mitotic compounds, specifically paclitaxel and peloruside. If successful, it is expected that these antimitotic compounds will halt proliferation of autoreactive T cells, either permanently or transiently, resulting in the full prevention or a delayed onset of EAE.

Additionally, it is theorised that most of the cells of the immune system will remain intact, thus the drugs will have a minimal overall impact on immune system function. 


\section{Chapter 2: General Methods}

\subsection{Animals, Maintenance, and Ethical Guidelines}

All animals were housed and maintained in the animal facilities at Victoria University of Wellington, New Zealand, under the guidelines specified by the Ministry of Agriculture and Forestry (MAF). Mice were raised in filter-top cages in temperature-regulated facilities, with maintenance and litter changes performed at regular intervals. Experimental protocols were approved by the Victoria University of Wellington Animal Ethics Committee (AEC) under the licenses 2002R6 and 2004R17.

Originally purchased from the Jackson Laboratory (Bar Harbor, ME, USA), C57BL/6 mice were bred at Wellington School of Medicine, Wellington, New Zealand. Transgenic mice, including toll-like receptor-4 knockout (TLR4 -/-), TLR2 -/-, and 2D2 (H-2 $)$ TCR mice, were generously donated by Thomas Bäckström of the Malaghan Institute of Medical Research, Wellington, New Zealand. TLR4 -/mice are deficient in the major LPS receptor, TLR4; TLR2 -/- mice lack TLR2; and 2D2 transgenic mice have a majority proportion of their $\mathrm{CD} 4^{+} \mathrm{T}$ cells with a TCR specificity for $\mathrm{MOG}_{35-55}$ (Bettelli et al., 2003). All mice were bred on a C57BL/6 background.

\subsection{Reagents}

Paclitaxel (purified from Taxus yannanensis), cyclosporin A, and LPS (from E. coli) were purchased from Sigma Chemical Co. (St. Louis, MO). Taxol ${ }^{\circledR}$ (Taxol; Bristol- 
Myers Squibb, New York City, NY) was purchased from Capital Coast Chemists (Wellington, New Zealand). Peloruside, mycalamide, and pateamine were generously provided by Dr. Peter Northcote (Victoria University of Wellington, School of Chemical and Physical Sciences). Paclitaxel, peloruside, mycalamide, pateamine, and cyclosporin A were dissolved in ethanol and stored as $1 \mathrm{mM}$ stocks (10 mM stock for paclitaxel) at $-80^{\circ} \mathrm{C}$.

\subsection{Primary Cell Preparation and Culture}

\section{Dissections/Organ Removal}

Animals used for cell preparation were euthanised in a $\mathrm{CO}_{2}$ chamber and, after death was confirmed, immediately transferred to a dissecting table. Animals were washed with $100 \%$ ethanol before surgery. Sterility of surgical tools was maintained by storage in $100 \%$ ethanol during organ removal. The thorax was opened for the removal of the draining lymph nodes (DLN) and bones. The peritoneal cavity was opened for the removal of internal organs and tissues such as the spleen (SPL), liver, kidneys, intestine (INT), mesenteric lymph nodes (mLN), and blood (by cardiac puncture). Bones, organs, and other tissues were removed by blunt dissociation of connective tissue. Blood was obtained by cardiac ventricular puncture and extraction with a 27.5 -ga needle and syringe.

The brain and spinal cord were removed following decapitation. The brain was carefully dissected out of the skull. The spinal cord was removed by pressurised extraction. Briefly, a pre-filled syringe containing PBS with an attached $19 \mathrm{~g}$ needle was inserted into the lumbar region of the vertebral column. After removal of the head in the cervical region, positive pressure was exerted on the syringe, forcing the 
spinal cord out of the vertebral column via the cervical opening. The brain, as well as all other organs except for blood, were transferred to tubes containing either zinc fixative (see Appendix A) or ice-cold wash media (see Appendix A) for cell preparation and culture.

\section{Macrophages}

Bone marrow-derived macrophages (BMM $\varnothing$ ) were cultured from C57BL/6 mice as previously described (Celada et al., 1984). Briefly, long bones were flushed with sterile divalent cation-free Dulbecco's PBS (dPBS) (Invitrogen, Auckland, New Zealand) with a 23-ga needle. Cells were cultured in complete medium (CTCM; see Appendix A) in the presence of GM-CSF and IL-3 (from the supernatants of GMKLON and WEHI-3 cell lines, respectively). The cells were cultured for $24 \mathrm{hr}$ at $37^{\circ} \mathrm{C}$ in air with $5 \% \mathrm{CO}_{2}$. Non-adherant cells were removed and further cultured for 7-10 days at $37^{\circ} \mathrm{C}$ in air with $5 \% \mathrm{CO}_{2}$ to allow cells to differentiate into macrophages. Once a monolayer of cells was established, BMM $\varnothing$ were removed by vigorous pipetting, and $10^{5}$ cells $/ \mathrm{ml}$ were resuspended in CTCM. Cells were then

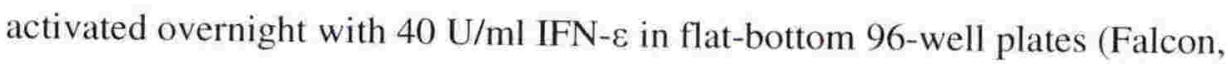
Franklin Lakes, $\mathrm{NJ}$ ) at $37^{\circ} \mathrm{C}$ in air with $5 \% \mathrm{CO}_{2}$.

\section{Splenocytes and Lymph Nodes}

Single cell suspensions of splenocytes and lymph node (LN) cells were enriched by dissociation of the SPL or LNs through sterile $70-\mu \mathrm{m}$ cell strainers (Falcon) into 50 $\mathrm{ml}$ polypropylene centrifuge tubes (Brunet et al., 1997). For splenocytes, red blood cells were lysed with red blood cell lysis buffer (Sigma). Cells were resuspended in 
CTCM and cultured at $37^{\circ} \mathrm{C}$ in air with $5 \% \mathrm{CO}_{2}$ in flat-bottom 96-well plates (Falcon) $\left(10^{6}\right.$ cells/well).

\subsection{EAE Immunisations}

To induce EAE, 8-12 week old C57BL/6 mice were immunised with an emulsion of antigen in CFA consisting of 50 ع $\mathrm{g} \mathrm{MOG}_{35-55}$ peptide (Mimotopes; Clayton, Vic, Australia), $500 \varepsilon \mathrm{g}$ heat-inactivated Mycobacterium tuberculosis (Difco Laboratories; Detroit, MI), $100 \varepsilon 1 \mathrm{dPBS}$, and $100 \varepsilon 1 \mathrm{IFA}$ (Sigma). A total of $200 \varepsilon 1$ was injected s.c. in the hind flanks of each mouse. In addition, each mouse was injected i.p. with 200 ng pertussis toxin (PTx) (Sapphire Bioscience; Redfern, NSW, Australia) in specialised PTx buffer (see Appendix A) at the time of initial immunisation and again $48 \mathrm{hr}$ later. The weight of each mouse was recorded daily, as well as observations made for any signs of clinical disease, from the day of immunisation until the day they were euthanised. The onset of EAE followed a typical pattern in which paralysis began from the tail and gradually worked its way up the caudalrostral axis. To quantify the level of disease, severity was measured by a typical field scoring system:

1 = Half tail paralysis; loss of tail tonicity $2=$ Full tail paralysis

$3=$ One hind limb paralysis, or severe weakness in both hind limbs

$4=$ Full hind limb paralysis

$5=$ Hind limb and front limb paralysis; moribund 
Scores were recorded daily by the same individual in order to reduce subjective variation. For ethical reasons, animals were euthanised if found to be in a moribund state.

\subsection{Measuring Body Temperature, Weight, and Appearance of}

\section{Mice.}

Body temperatures, when required, were obtained by gently inserting a lubricated RET-3 rectal probe (ADInstruments) into the anuses of restrained mice.

Temperatures were recorded as ${ }^{\circ} \mathrm{C}$. Weights of the mice were measured on a Mettler PE 6000 balance (Mettler Toledo; Columbus, $\mathrm{OH}$ ). The activity and appearance of the mice were gauged on a pre-determined scale to uphold ethical standards throughout experimentation. Mouse activity was scored as follows:

$0=$ normal

$1=$ low activity/slightly weak

$2=$ little movement/limp to handle

3 = moribund

Mouse appearance was scored as follows:

$0=$ normal

1 = lack of grooming (slightly greasy coat)

$2=$ total lack of hygiene (very greasy coat, roughed up appearance)

Mice were euthanised if they had a $20 \%$ weight loss and a combined score of $\varepsilon 3$ for activity and appearance. 


\subsection{Enzyme-Linked Immunosorbant Assays for Cytokine Analysis}

A Pharmingen sandwich ELISA was used to measure cytokines produced by cells in culture following the manufacturer's recommendations (BD Pharmingen; Franklin Lakes, NJ). Briefly, supernatants from cell cultures were removed and stored at $20^{\circ} \mathrm{C}$. Samples were stored at $4^{\circ} \mathrm{C}$ if they were to be used on the day of the ELISA measurement. To prepare the ELISA plate (Greiner; Monroe, NC), a cytokinespecific capture antibody was diluted in capture buffer (see Appendix A) and transferred to 96 -well plates. The plates were then incubated at $4^{\circ} \mathrm{C}$ overnight. Wells were rinsed with Tween-PBS (TPBS). In order to block non-specific binding, wells were incubated for $2 \mathrm{hr}$ with PBS containing 5\% FCS. After a TPBS rinse, samples were added directly to the wells, or diluted with 5\% FCS in PBS solution, then added to the wells. After $2 \mathrm{hr}$, plates were washed in TPBS. Diluted biotinylated detection antibody was added immediately after the last wash, followed by a 1-hr incubation. Plates were re-washed in TPBS, followed by a 30-min incubation with the recommended dilution of streptavidin-HRP (SA-HRP). A final wash in TPBS was performed. Lastly, substrate reagents "A" and "B" were added to induce a color change that directly correlated to cytokine concentration, followed by the addition of $0.18 \mathrm{M}$ sulfuric acid to stop the reaction. Well absorbances were read on a VersaMax plate reader (Molecular Devices; Sunnyvale, CA, USA) at a wavelength of $450 \mathrm{~nm}$.

\subsection{Metabolism and Proliferation Assays}

\section{$\underline{\text { MTT colorimetric assay }}$}


MTT (3-(4,5-dimethylthiazol-2-yl)-2,5-diphenyltetrazolium bromide) is a tetrazolium salt that reduces to purple formazan in the presence of reducing compounds, such as NADH and NADPH (Berridge et al., 2005; Hansen et al., 1989; Mosmann, 1983). Dose-response curves were obtained by comparing drug-treated cells to untreated controls following the addition of MTT to cultures. Similarly, metabolic activity of non-proliferating cells, based on the relative presence of reducing compounds, was assayed with MTT in BMM $\varnothing$ cultures.

\section{$\underline{\text { CyQUANT proliferation assay }}$}

A CyQUANT kit (Invitrogen; Auckland, NZ) was used as an alternative assay to detect cell proliferation when total cell numbers were limited. This assay was performed following the manufacturer's recommendations. CyQUANT measures the total DNA content of a culture, which detects proliferation in cultures with low cells numbers $\left(<5 \times 10^{5}\right.$ total cells). Briefly, $2 \times 10^{5}$ cells were cultured in a 96-well plate with CTCM $\left(37^{\circ} \mathrm{C}\right.$ in air with $\left.5 \% \mathrm{CO}_{2}\right)$. After $72 \mathrm{hr}$, the supernatants were removed

and the plates containing the cells were stored at $-20^{\circ} \mathrm{C}$. To perform the assay, the $96-$ well plates were removed from the $-20^{\circ} \mathrm{C}$ freezer, followed by incubation with the CyQUANT lysis buffer. The detection reagent, a green-fluorescing dye that binds to cellular DNA, was then added to each well. The light absorbance of the 96-well plates were determined on a SpectraMax Gemini fluorescence plate reader (Molecular Devices; Sunnyvale, CA, USA) with excitation/emission wavelengths of $480 / 520 \mathrm{~nm}$, respectively. 


\subsection{General FACS Analysis}

Stained cells were examined in a FACScan flow cytometer (Becton Dickinson, Franklin Lakes, NJ). In brief, 10,000 cell events were collected for each assay, and the data were analysed by CellQuest Pro software (Becton Dickinson).

Fluorescently labeled antibodies (BD Pharmingen) were incubated with cells according to the manufacturer's recommendations. Each sample was stained with either a fluorescently labeled antibody or an isotype control to correct for autofluorescence and background staining.

\subsection{Histology}

The tissues/organs used for histology were removed from mice and were preserved in zinc fixative (Appendix A). Paraffin embedding, slide preparation, and hematoxylin and eosin (H\&E) staining were performed by the Pathology Department at the Wellington School of Medicine and Health Sciences, Wellington, New Zealand. Lesions in spinal cord tissue from mice were identified, measured, and counted. In addition, an average severity score was assigned for each lesion present in an affected spinal cord (Fig. 2.1):

$0=$ normal; no cellular infiltrates

$1=$ mild; $<50 \varepsilon \mathrm{m}$ diameter foci of infiltrating cells in the perivascular region.

$2=$ moderate; $50 \varepsilon \mathrm{m}-100 \varepsilon \mathrm{m}$ diameter foci in the perivascular region.

$3=\quad$ severe; large foci $(>100 \mathrm{\varepsilon m})$ in the CNS parenchyma

It was expected that this method of determining the severity of spinal cord inflammatory cell infiltration would be subjective, and therefore the individual 
mouse disease scores were not compared until lesion scoring was completed for all samples. It was also recognised that spinal cord lesions were 3-dimensional, thus measuring the size of the lesion foci in the particular dimension on the slide would not necessarily represent the actual size of the entire lesion; however, using this method, the relative sizes and abundance of lesions can be quantified and compared across all sample groups. 

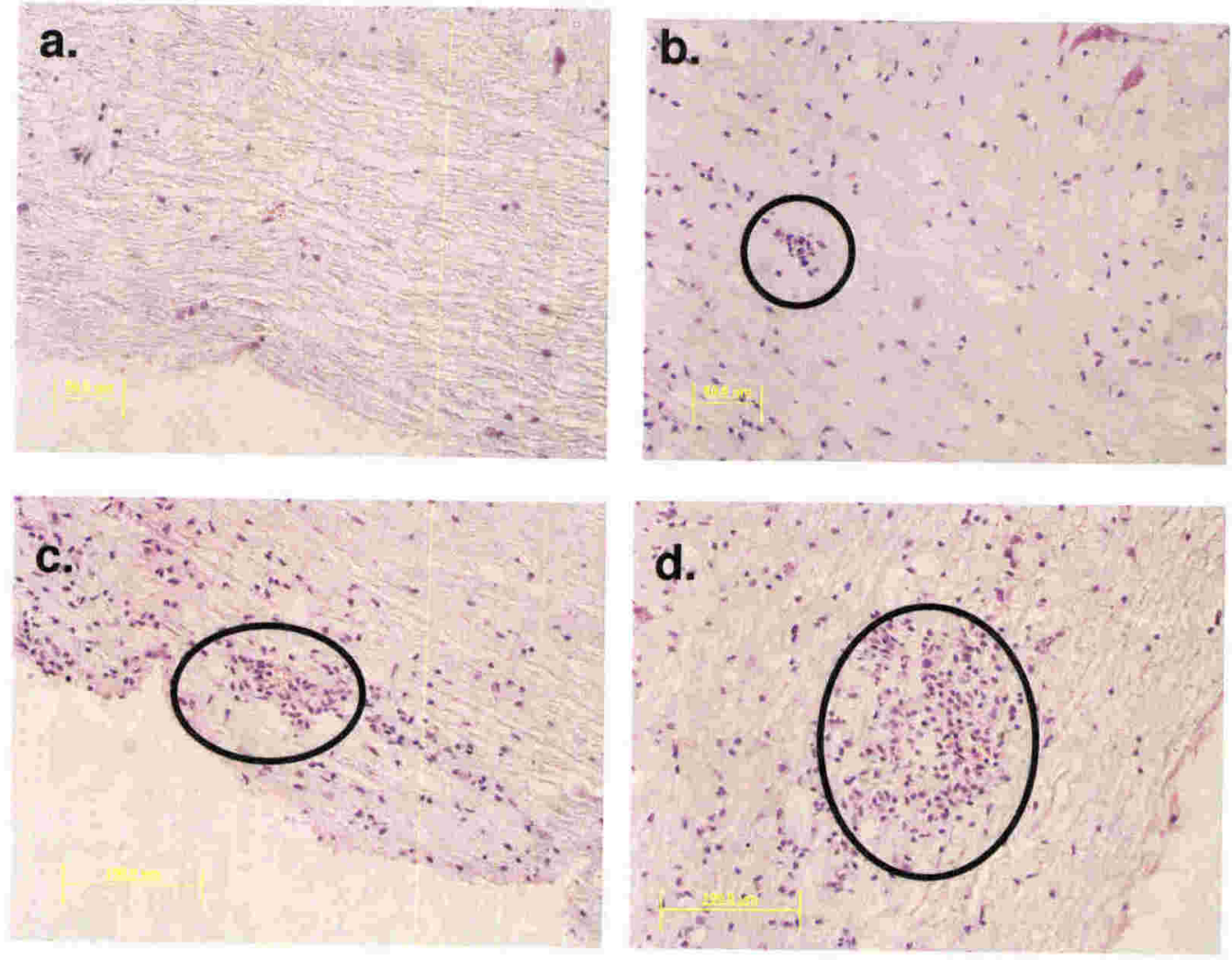

\section{Figure 2.1 Examples of infiltrating cell foci.}

Shown above are a normal spinal cord (a), a mild lesion (b), a moderate lesion (c), and a severe lesion (d). The yellow scale bars represent $50 \leq \mathrm{m}$ (a $\&$ b) or $100 \leq \mathrm{m}$ (c \& d).

For immunohistochemistry (IHC), paraffin-embedded tissues were deparaffinised and rehydrated. Slides containing the tissue were then blocked with $1 \%$ BSA in PBS, followed by incubation with a biotinylated antibody. $\mathrm{ABC}$ reagents (Vector Laboratories, Burlingame, CA) and DAB solution (Sigma) were used following the manufacturers' recommendations for antibody detection. DAB staining appears brown under a light microscope. Methyl green, which stains chromatin, was used as a counterstain for cellular nuclei. 


\subsection{Graphing and Statistical Analyses}

GraphPad Prism (Version 4.0, Graphpad Software, Inc.; San Diego, CA) was used to determine significance of data. SigmaPlot (SSPS Science; Chicago, IL) was used for data manipulations and graphing. All data points are expressed as the mean \pm standard error unless otherwise noted. 


\section{Chapter 3: Immunomodulatory Effects of the Anti-}

\section{Mitotic Compounds, Paclitaxel and Peloruside.}

\subsection{Introduction}

Pateamine, mycalamide, and peloruside are cytotoxic compounds isolated from Mycale hentsheli (West et al., 2000). Previous studies have suggested that mycalamide and pateamine exhibit immunosuppressive properties (Galvin et al., 1993; Romo, 1998; Romo et al., 2004); however, Hood et al. (2002) showed that pateamine and mycalamide were not directly immunosuppressive in a mixed lymphocyte reaction (MLR). While few studies have investigated the immunomodulatory activities of peloruside, Hood et al. (2002) showed that peloruside non-specifically reduced IL-2 production from lymphocytes by inhibiting proliferation in a MLR. In these studies, there was no mention of the lymphocyte subsets that were directly affected by peloruside.

Paclitaxel has a long clinical history in the treatment of cancer. Paclitaxel is known to be cytotoxic to proliferating splenocytes and cancer cells at concentrations in the nM range in vitro (Brown et al., 1985; Horwitz et al., 1993). Another property of paclitaxel is that it mimics bacterial lipopolysaccharide (LPS) in murine macrophages and DCs by binding to the TLR4 complex (Byrd-Leifer et al., 2001; Ding et al., 1993; Joo, 2003). The LPS-mimicry observed with paclitaxel is detectable in vitro at concentrations above $1 \varepsilon \mathrm{M}$, and suggests that paclitaxel may play an immunomodulatory role in vivo. 


\section{$\underline{\text { Aims }}$}

Peloruside, as well as the structurally unrelated compounds mycalamide and pateamine, are cytotoxic agents isolated from a Mycale sp. of marine sponge (West et al., 2000). Mycalamide and pateamine have been identified as having immunosuppressant activities (Galvin et al., 1993; Romo, 1998; Romo et al., 2004). To confirm whether these 3 compounds exhibit immunosuppressive activities, peloruside, pateamine, and mycalamide were compared to a known immunosuppressant, cyclosporine A, to clarify the nature of immunosuppression in all of these compounds.

Although the mechanism of microtubule-stabilisation has been elucidated for paclitaxel and peloruside (Bhalla, 2003; Donaldson et al., 1994; Fuchs and Johnson, 1978; Gaitanos et al., 2004; Gligorov and Lotz, 2004; Hood et al., 2002; Manfredi et al., 1982; Rowinsky et al., 1988; Schiff et al., 1979; Schiff and Horwitz, 1980), there are very few studies looking at other bioactive roles for peloruside, specifically whether or not it induces a pro-inflammatory response similar to paclitaxel. Therefore, in order to understand how peloruside impacts on cells of the immune system, the following set of experiments was designed to compare the effects of paclitaxel to peloruside in murine $M \varnothing$ and splenocytes. This includes identifying immune cell populations susceptible to the anti-mitotic effects of both compounds, as well as assaying for immunostimulation. 


\subsection{Methods}

\subsubsection{Anti-CD3 $\mathrm{T}$ cell receptor stimulation}

Splenocytes from C57BL/6 mice were isolated as described in Chapter 2. T cells were activated by cross-linking of TCRs to plate-bound anti-CD3 $\varepsilon$ antibody ( $\varepsilon$-CD3) (0.5 ع g/well) (hamster mAb 145-2C11, BD Bioscience, Franklin Lakes, NJ). Cells were immediately cultured in the presence of peloruside, mycalamide, pateamine, or cyclosporine A at a range of concentrations for $24 \mathrm{hr}$ or $72 \mathrm{hr}$ (in the case of mycalamide and cyclosporine A). Cultured cells in triplicate wells were pooled for FACS analysis.

\subsubsection{FACS analysis}

FACS analyses were performed as described in Chapter 2.8. In brief, $\mathrm{CD} 4^{+}$and $\mathrm{CD}^{+}$T cells were identified by fluorescently-labeled antibodies (BD Pharmingen). Cells were stained with annexin-V:FITC (Roche; Germany), which binds phosphatidylserine, and the DNA-binding salt propidium iodide (PI) (Sigma) prior to FACS analysis. Together, these stains act as apoptotic markers. Cellular debris was gated out based on a size threshold (see Appendix C). Each test sample was accompanied by a time-matched control sample (cells not treated with drug).

\subsubsection{Stimulation of $\mathbf{T}$ cells in splenocyte cultures}

Splenocytes were isolated from the spleens of C57BL/6 mice (described in Chapter 2). Cells $\left(10^{6} /\right.$ well) were cultured in a 96 -well plate (Falcon) with $3 \varepsilon \mathrm{g} / \mathrm{ml}$ of the T cell mitogen, concanavalin A (Con A; Sigma), and dilutions of peloruside or paclitaxel. After a 72-hr incubation period (conditions described in Chapter 2), a MTT proliferation assay was performed on the cells. 


\subsubsection{Assessment of cell viability, metabolism, and proliferation}

Viability of $\varepsilon$-CD3-stimulated splenocytes was determined by FACS analysis and expressed as the number of gated annexin- $\mathrm{V}^{\text {neg }} \mathrm{CD} 4^{+} / \mathrm{CD}^{+}$cells divided by the total number of cells compared to an untreated, time-matched control (see Appendix C). Metabolism and proliferation were determined by the MTT assay (described in Chapter 2).

\subsubsection{IFN- $\alpha$, TNF- $\alpha$, IL-12, IL-10 and NO detection}

All ELISAs were performed following the manufacturer's recommendations (see Chapter 2) (BD Pharmingen). Anti-mouse antibodies against IFN- $\varepsilon$, TNF- $\varepsilon$, IL12p40, and IL-10 (both capture and detection) were raised in rats, and none show any known cross reactivity to other cytokines. All ELISAs were performed within the recommended linear detection limits of the respective kits. The accumulation of $\mathrm{NO}_{2}^{-}$, an indicator of $\mathrm{NO}$ production in the culture medium, was assayed by the Greiss reaction (Green et al., 1982).

\subsection{Results}

\subsubsection{The effect of mycale sponge metabolites on $\mathrm{T}$ cell receptor signaling.}

Metabolites from Mycale hentsheli, including peloruside, mycalamide and pateamine, were tested for immunosuppressive activities alongside the known immunosuppressant, cyclosporin A. Splenocytes were stimulated by plate-bound $\varepsilon$ CD3 antibody, which acts as a CD3 agonist, and bypasses the need for MHC plus specific peptide. The MTT cell proliferation assay was used to assess effects of the compounds on T cell proliferation, and annexin-V/PI binding was used to determine 
the proportion of viable, non-apoptotic cells. Annexin-V binds to phosphatidylserine, which is normally on the inner layer of the plasma membrane, but during the early stages of apoptosis, phosphatidylserine translocates to the external membrane of the cell where it is accessible for binding to annexin-V. The use of PI, which binds to DNA, differentiates the cells with membrane damage from apoptotic cells since PI will only stain DNA if there is membrane disruption such as occurs during necrosis, or if the cell is in late apoptosis. IFN- $\gamma$ production was used as a marker of TCRstimulated cytokine production.

Peloruside and pateamine inhibited $\varepsilon-\mathrm{CD} 3$-stimulated T cell proliferation and IFN- $\varepsilon$ production (Fig. 3.1 a-b), but did so by causing apoptosis. Unfortunately, however, this particular stock of peloruside was discovered to contain a highly cytotoxic contaminant (see Appendix B for details); therefore, Fig. 3.1 a was erroneous data. In the case of mycalamide and cyclosporin A, however, IFN-E production was completely inhibited at the higher concentrations tested, with $\mathrm{T}$ cell viability never falling below $30 \%$ of the untreated controls after a 24 -hr treatment (Fig. 3.1 c-d). Mycalamide inhibited cell proliferation to a greater extent than cyclosporin A. Longer exposures of $72 \mathrm{hr}$ completely inhibited cell proliferation (MTT assay); however, cell viability remained high (36\% for mycalamide at $10 \mathrm{nM}$ and $28 \%$ for cyclosporin A at $1000 \mathrm{nM}$ ) (Fig. 3.1 e-f). This suggests that mycalamide, like cyclosporin $\mathrm{A}$, has immunosuppressive properties, defined by reduced proliferation and IFN- $\varepsilon$ production without causing cell death. Although peloruside results were negated, the other sponge metabolites and cyclosporin A are valid, and are shown because they are metabolites from the same sponge and may have a direct effect on $\mathrm{T}$ 
cell activation. All further peloruside experiments were performed with a different, non-contaminated stock of peloruside.

To identify if there was selective inhibition of lymphocytes, software-generated selectivity gates were placed on $\mathrm{CD} 4^{+}$and $\mathrm{CD} 8^{+} \mathrm{T}$ cells in FACS analyses (see Appendix $\mathrm{C}$ for gating). The respective viabilities of the two $\mathrm{T}$ cell subsets were similar (data not shown), and the cells were therefore grouped for analysis purposes. 


\section{Anti-CD3}

\section{stimulation}
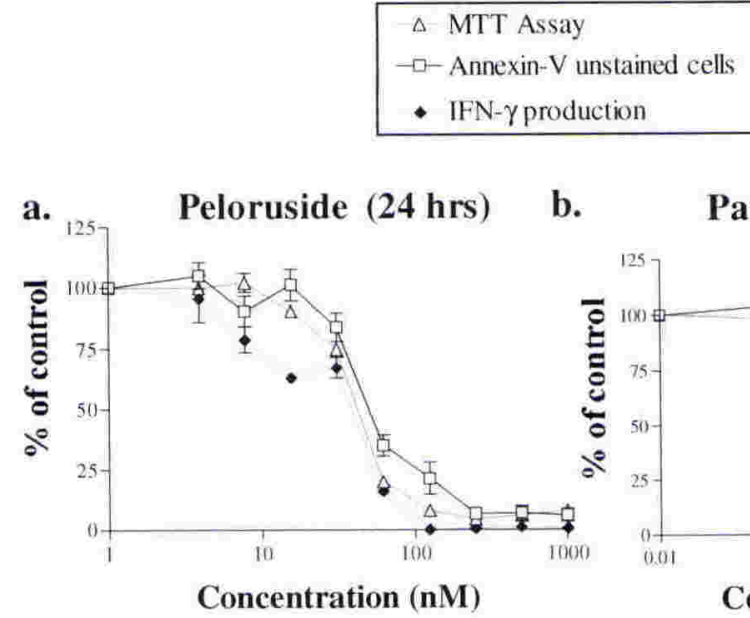

b. Pateamine (24 hrs)

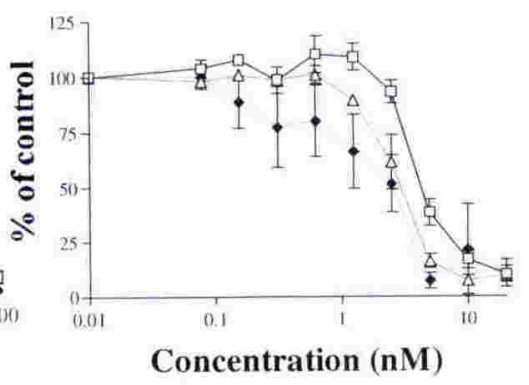

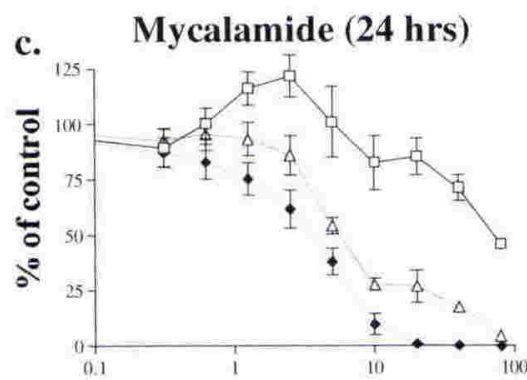

Concentration (nM)

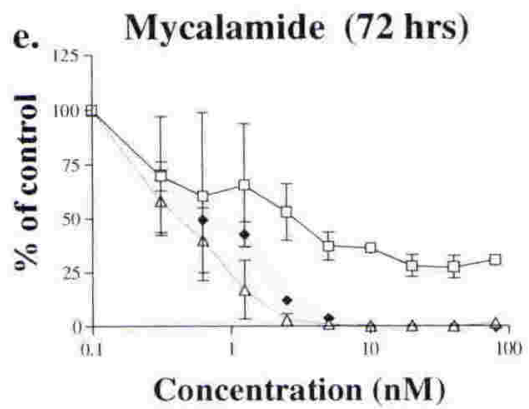

d. Cyclosporin A (24 hrs)

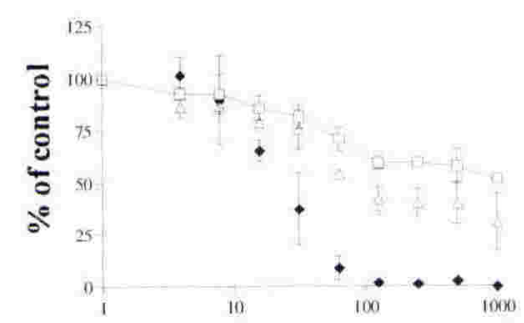

Concentration $(\mathrm{nM})$

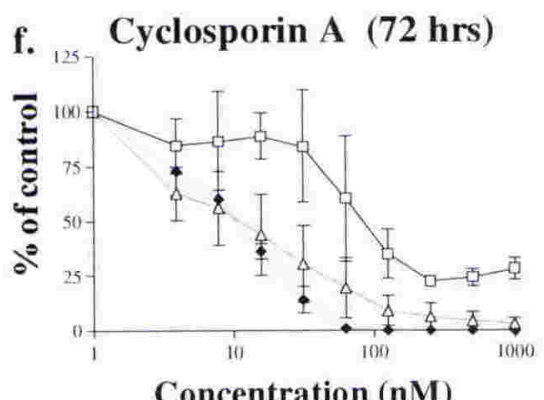

Figure 3.1 Anti-CD3-stimulated $\mathrm{T}$ cell responses to sponge metabolites and cyclosporin A. Isolated spleen cells were stimulated to divide by cross-linking to $0.5 \mu \mathrm{g} / \mathrm{ml} \leq$-CD3 antibody per well of a 96-well plate, immediately followed by treatment with cytotoxic drugs. T cell proliferation (open triangles) was measured by the MTT assay after 24 or $72 \mathrm{hr}$ of culture. T cell viability, assessed as the annexin $\mathrm{V}$-unstained proportion (open squares), was analysed by flow cytometry. IFN- $\leq$ (filled diamonds) was determined by ELISA, and the mean and SEM of 3 separate experiments performed in triplicate are presented. All results are expressed as the $\%$ of an untreated control. Peloruside data (a) is erroneous due to contamination. 


\subsubsection{Peloruside and paclitaxel are cytotoxic to proliferating splenocytes.}

Using the MTT cell proliferation assay, $72-\mathrm{hr} \mathrm{IC}_{50}$ values of peloruside and paclitaxel were measured in proliferating splenocytes to determine if these compounds can inhibit $\mathrm{T}$ cell proliferation without being cytotoxic to unstimulated cells. Paclitaxel and peloruside were cytotoxic to Con A-stimulated splenocytes with $\mathrm{IC}_{50}$ values of $30 \mathrm{nM}$ and $83 \mathrm{nM}$, respectively (Fig. 3.2 a). The $\mathrm{IC}_{50}$ values for Con A-stimulated splenocytes were determined by nonlinear regression. The minimum absorbance values were as low as $40 \%$ of the control, but never reached $0 \%$. This is likely due to the nature of Con A stimulation. T cells comprise about half of splenocytes and Con A is a T cell mitogen, which leaves many cells in a splenocyte population unstimulated, thus not susceptible to the anti-mitotic effects of peloruside and paclitaxel Little to no cytotoxicity was observed in cultures of unstimulated splenocytes treated with either drug (Fig. 3.2 b).

\subsubsection{Peloruside does not induce the release of pro-inflammatory cytokines by ВММØ.}

Because paclitaxel, in addition to its microtubule stabilising effects, can mimic LPS in thioglycolate-elicited peritoneal murine macrophages and cause the release of proinflammatory cytokines (Byrd-Leifer et al., 2001), BMMØ were cultured with paclitaxel or peloruside at a wide range of concentrations to assess whether peloruside has similar LPS mimetic properties to paclitaxel. In agreement with the literature, paclitaxel caused the production of IL-12p40 and NO in IFN-E-activated BMM $\varnothing$ (Fig. $3.3 \mathrm{a}$ and b). Surprisingly, no TNF- $\varepsilon$ was detectable at $8 \mathrm{hr}$ in the supernatants of BMM $\varnothing$ when cultured with a range of paclitaxel concentrations up to 10 \&M (Fig. 3.3 c); however, TNF- $\varepsilon$ was detected at $2 \mathrm{hr}$ from cultures containing 10 
عM paclitaxel (Fig. 3.3 d). In BMM $\varnothing$ cultured with peloruside, there was no IL12p40, NO, or TNF- $\varepsilon$ detected. Additionally, the anti-inflammatory cytokine IL-10 was not detected in supernatants of either peloruside- or paclitaxel-treated cells (data not shown).
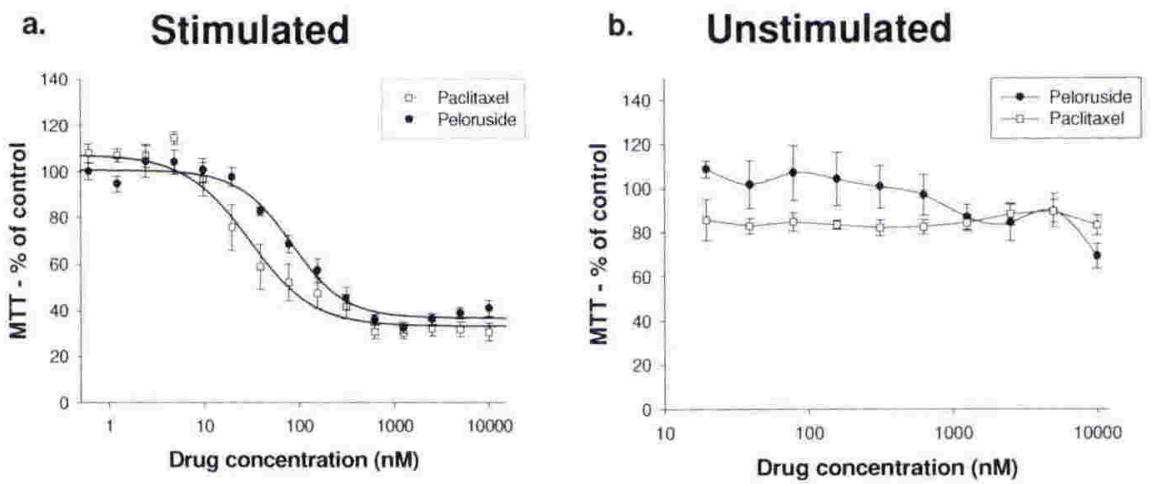

\section{Figure 3.2 Determining $I C_{50}$ values of peloruside and paclitaxel in} splenocytes. Peloruside (filled circles) or paclitaxel (open squares) was cultured for $72 \mathrm{hr}$ with splenocytes that were stimulated with $3 \mathrm{sg} / \mathrm{ml}$ Con-A (a) or unstimulated (b). Both peloruside and paclitaxel inhibited proliferation of stimulated splenocytes (a) with little to no cytotoxicity in unstimulated splenocytes (b). Data points are representative of duplicate or triplicate wells from 3 - 4 experiments.

LPS stimulates BMM $\varnothing$ primarily through toll-like receptor-4 (TLR4), resulting in the release of pro-inflammatory cytokines. To confirm that paclitaxel was acting directly on the TLR4 complex in its LPS mimicry, these experiments were repeated on BMM $\varnothing$ from TLR4 -/- and TLR2 -/- mice (Figs. 3.4 and 3.5). Not surprisingly, the absence of TLR4 on BMM $\varnothing$ cultured with paclitaxel (Fig. 3.4) completely 
ablated cytokine production. There was no difference in the production of inflammatory mediators by TLR2 -/- and wild-type BMMØ in response to paclitaxel (Fig. 3.4), and again, peloruside had no effect on IL-12, NO, or TNF- $\varepsilon$ production by TLR2 -/- or TLR4 -/- BMMØ (Fig. 3.5). To determine whether the absence of cytokines in supernatants of BMM $\varnothing$ cultured with peloruside was due to general cytotoxicity, the viability of drug-exposed BMM $\varnothing$ was assayed by MTT reduction. After a 72-hr incubation, neither peloruside (Fig. 3.5 d) nor paclitaxel (Fig. 3.4 d) were cytotoxic to BMMØ. Interestingly, concentrations of paclitaxel in the $\varepsilon \mathrm{M}$ range produced up to a 2-fold increase in MTT reduction in wild-type and TLR2-/-cells, indicating a large increase in reducing compounds such as NADH and NADPH within these BMM $\varnothing$, thus suggesting enhanced metabolic activity. This enhanced activity was not seen in splenocytes (Fig. 3.3). These results indicate that, although paclitaxel and peloruside share a similar anti-mitotic mechanism of microtubule stabilisation, peloruside does not share the LPS mimicry of paclitaxel. 
a.

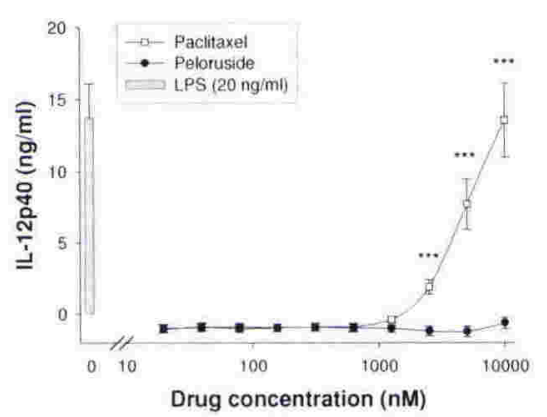

c.

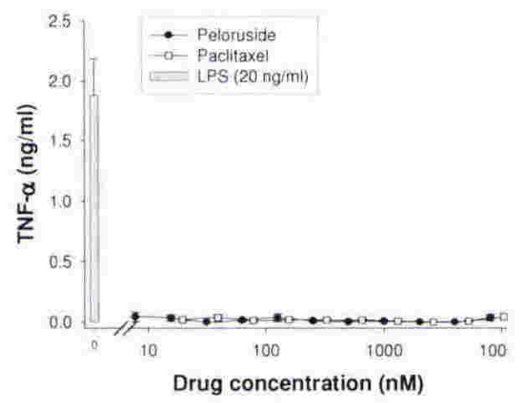

b.

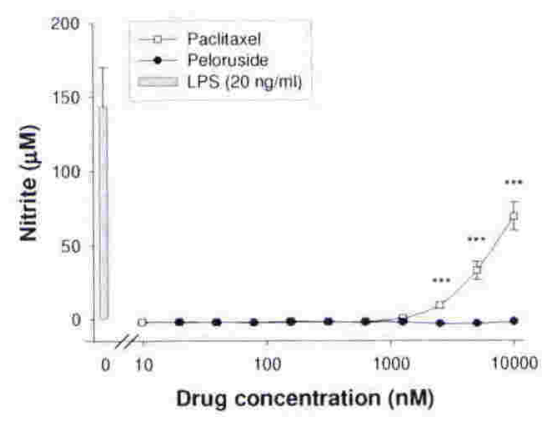

d.

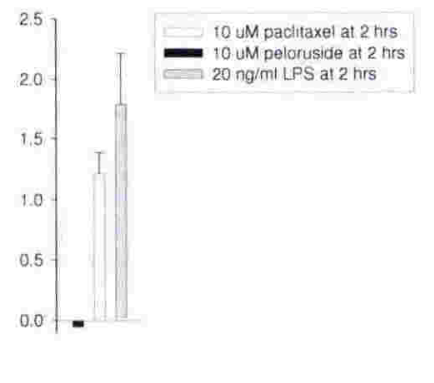

Figure 3.3 Effect of peloruside and paclitaxel on BMMØ. IFN- $\leq-$ activated BMM $\varnothing\left(5 \times 10^{4}\right.$ cells/well) were cultured with either peloruside $(\leq)$ or paclitaxel ( $\leq$ ) at a range of concentrations. The response to LPS (20 ng/ml) on its own is presented for comparison. Paclitaxel, but not peloruside, induced the production of IL-12p40 (a), nitrite (b), and TNF$\leq$ (d) from BMM $\varnothing$. No TNF- $\leq$ was detected from BMM $\varnothing$ after $8 \mathrm{hr}$ culture with paclitaxel or peloruside (c); however, TNF- $\leq$ was present in paclitaxel-treated cultures at $2 \mathrm{hr}$ (d). $\mathrm{NO}$ and cytokine levels were determined as described in Materials and Methods. Results are representative of duplicate or triplicate wells from 4 - 8 experiments. ${ }^{* \star *}$ $\mathrm{P}<0.001$ compared to no drug, as determined by the Student's $t$-test with a sequential Bonferroni correction. 


\subsubsection{Both peloruside and paclitaxel cultured with BMMØ decrease levels of}

TNF- $\alpha$ and NO production in the presence of LPS.

To determine if paclitaxel or peloruside could alter LPS-induced pro-inflammatory cytokine production, both compounds were added to cultures of LPS-stimulated BMMØ. When cultured with either paclitaxel or peloruside under these conditions, decreased levels of TNF- $\varepsilon$ and, to a lesser extent, NO were observed compared to a control with LPS alone (Fig. 3.6 a-b). The observed effect of decreased TNF- $\varepsilon$ and NO production was only seen at LPS concentrations less than or equal to $20 \mathrm{ng} / \mathrm{ml}$. When BMM $\varnothing$ were cultured with LPS concentrations higher than $20 \mathrm{ng} / \mathrm{ml}$, the antiinflammatory effects of the two drugs were no longer observed (data not presented). Interestingly, while peloruside and paclitaxel decreased TNF-E and NO production from LPS-stimulated BMMØ, neither drug had any obvious effect on IL-12p40 production from these same cells (Fig. 3.6 c). Finally, similar to previous experiments (Figs. $3.4 \mathrm{~d}$ and $3.5 \mathrm{~d}$ ), BMM $\varnothing$ treated with high concentrations of paclitaxel showed an enhanced metabolic activity above the levels seen with LPS alone (Fig. 3,6 d). 
a.

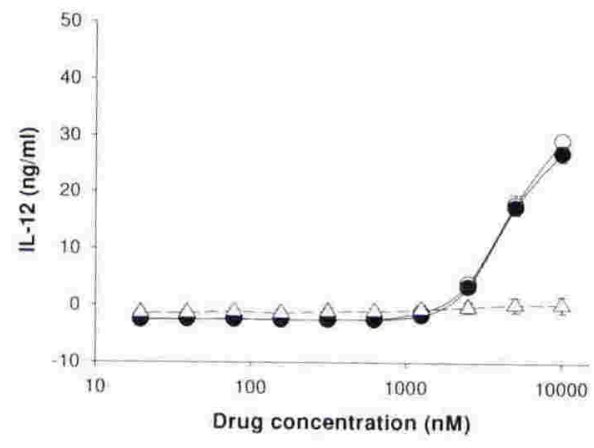

c.

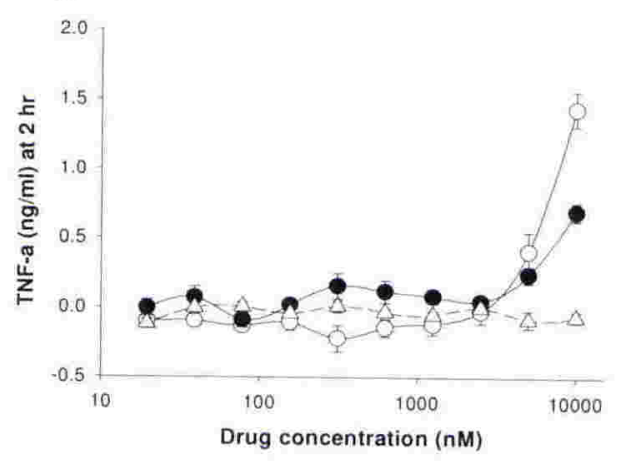

b.

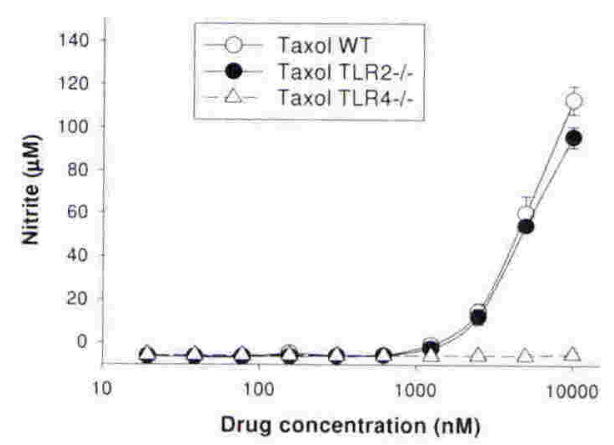

d.

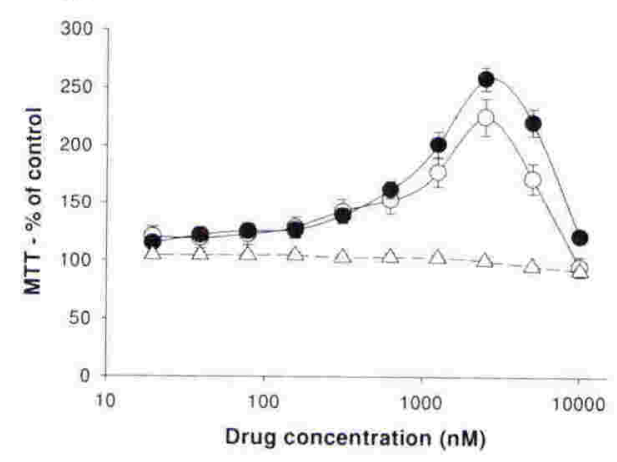

Figure 3.4 Paclitaxel in BMMØ.

A range of paclitaxel concentrations were cultured with IFN- $\leq-$ stimulated BMMØ from wild-type (open circles), TLR2 -/- (closed circles), and TLR4 -/- mice (open triangles). Supernatants from these cultures were removed and concentrations of IL-12 after $8 \mathrm{hr}(\mathrm{a})$, nitrite after $72 \mathrm{hr}$ (b), and TNF- $\leq$ after $2 \mathrm{hr}$ (c) were determined. A MTT assay after $72 \mathrm{hr}$ was performed to determine cell metabolism (d). Results are representative of triplicate samples from 3 experiments. 
a.

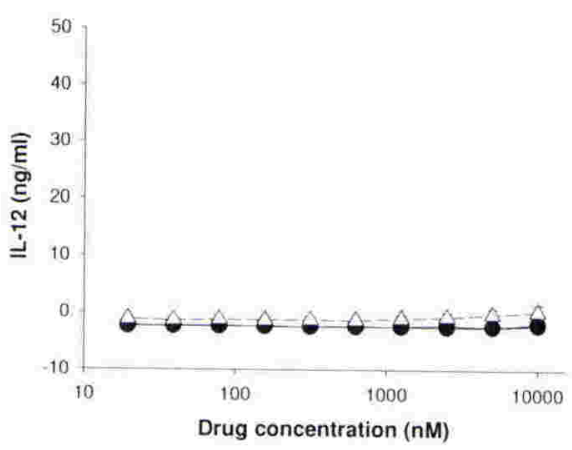

c.

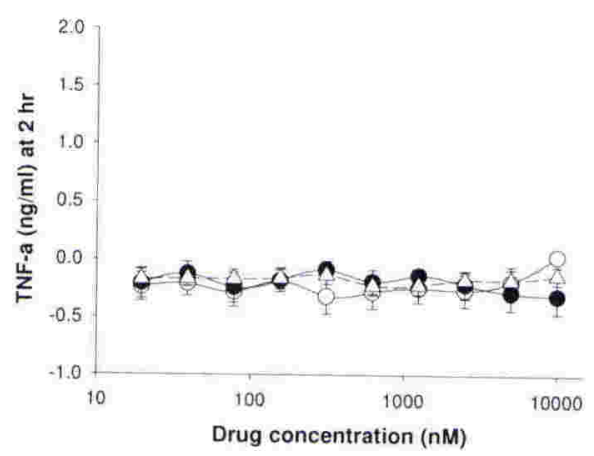

b.

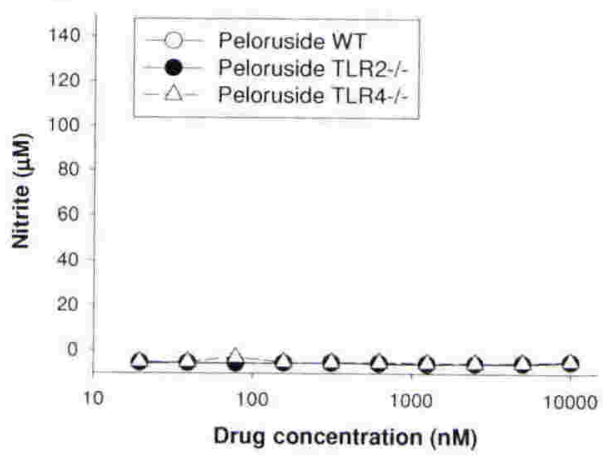

d.

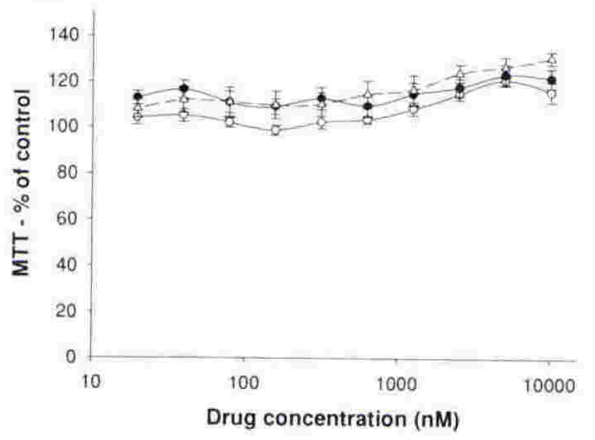

\section{Figure 3.5 Peloruside in BMMØ.}

Similar to Figure 3.4, IFN-S-stimulated BMM $\varnothing$ from wild-type (open circles), TLR2 -/- (closed circles), and TLR4 -/- mice (open triangles) were cultured with a range of peloruside concentrations. Supernatants from these cultures were removed and concentrations of IL-12 after $8 \mathrm{hr}$ (a), nitrite after $72 \mathrm{hr}$ (b), and TNF-s after $2 \mathrm{hr}$ (c) were determined. A MTT assay after $72 \mathrm{hr}$ was performed to determine cell metabolism (d). Results are representative of triplicate samples from 3 experiments. 
a.

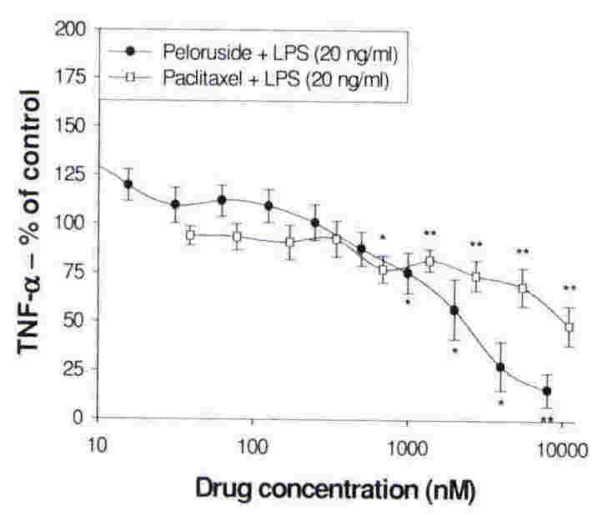

c.

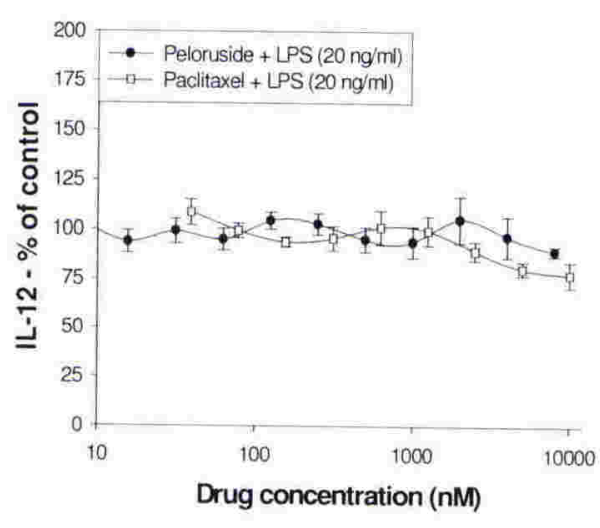

b.

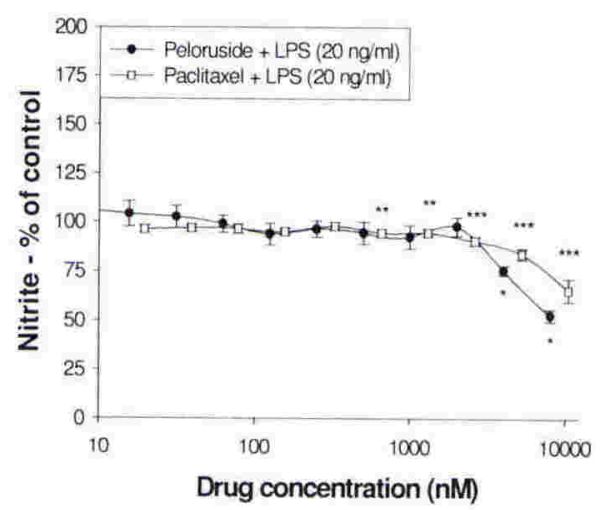

d.

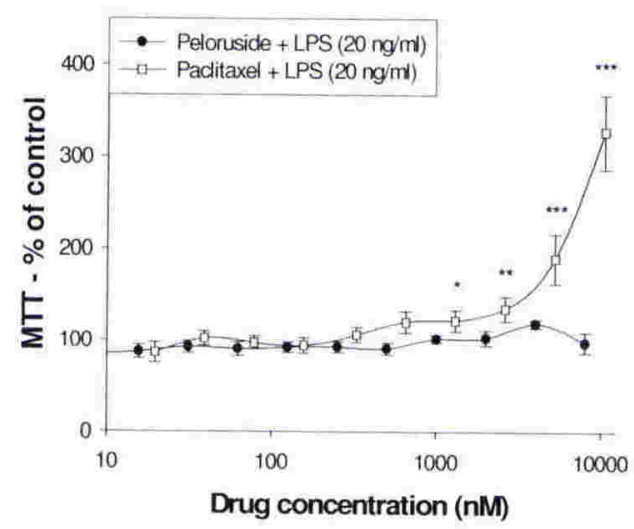

Figure 3.6 Effect of peloruside and paclitaxel in LPS-stimulated BMMØ.

BMMØ from wild-type mice were cultured with $20 \mathrm{ng} / \mathrm{ml}$ LPS and either peloruside $(\leq)$ or paclitaxel $(\leq)$ at a range of concentrations. Both compounds caused a significant decrease in levels of TNF- $\leq$ (a) and nitrite (b) at higher concentrations. IL-12p40 was unaffected by either drug (c). Paclitaxel also enhanced metabolic activity (d). Cytokine levels were determined by ELISA after $8 \mathrm{hr}$ incubation. NO (b) was determined by Greiss reaction after $72 \mathrm{hr}$. The MTT assay (d) was performed as described in Materials and Methods after $72 \mathrm{hr}$. All values are expressed as a \% of drug-free LPS-stimulated control BMM $\varnothing$.

Results are representative of duplicate or triplicate wells from 3 - 5 experiments. ${ }^{*} \mathrm{P}<0.05,{ }^{* *} \mathrm{P}<0.01$, ${ }^{* *} \mathrm{P}<0.001$, compared to LPS stimulation on its own, as determined by the Student's $t$-test with a sequential Bonferroni correction. 


\subsection{Discussion}

\subsubsection{Immunosuppressive properties of sponge metabolites}

Peloruside inhibited IFN- $\varepsilon$ production from $\varepsilon$-CD3-stimulated $\mathrm{T}$ cells, primarily by causing apoptosis. Later experiments, however, determined that this peloruside stock had a potent cytotoxic contaminant (Appendix B), therefore invalidating these results with peloruside. Interestingly, pateamine was not found to be immunosuppressive as previously reported (Romo, 1998; Romo et al., 2004). The relevance of pateamine to this project is limited, other than its use as a control cytotoxic compound from the same source as peloruside. Pateamine has been found to be a protein synthesis inhibitor that blocks the function of the initiation factor, eIF4A (Bordeleau et al., 2005). Mycalamide and cyclosporin A, although immunosuppressive in their mode of action, are also used as controls in the present study, mycalamide as another nonmicrotubule stabilizing cytotoxin and cyclosporin A as a true anti-inflammatory agent. Mycalamide, like pateamine, is a protein synthesis inhibitor but interacts directly with the ribosome (Burres and Clement, 1989).

One previous study looked at possible immunosuppressive effects of peloruside using a one-way MLR (Miller et al., 2004). The MLR is a proliferation assay that takes advantage of immune responses from allotypic cells. The responding $\mathrm{T}$ lymphocytes will proliferate in response to foreign peptides displayed on the allotypic MHC molecules located on APCs of the stimulator population. This method of stimulation is selective, in that the only T lymphocytes that respond are those with specific TCRs to the foreign peptides or those capable of recognising the allotypic MHC, a reactive subset that is normally between $1-10 \%$ of the total $\mathrm{T}$ cell population. This experiment provided valuable information on anti-proliferative 
responses of peloruside, but did not identify specific subsets of lymphocytes that were affected by the compound.

The method employed here used $\varepsilon-\mathrm{CD} 3$ antibody stimulation of splenocytes. This causes nearly all $\mathrm{T}$ cells in the mixed population of splenocytes to proliferate due to the direct stimulation of the TCR via $\varepsilon$-CD3, eliminating the need for APCs and decreasing the variability often seen in the MLR. It was hoped that the data generated from this experiment would determine if there is a specific immunosuppressive effect in $\mathrm{CD}^{+}$or $\mathrm{CD} 8^{+} \mathrm{T}$ cells in response to peloruside. Gates for $\mathrm{CD} 4^{+}$and $\mathrm{CD} 8^{+}$cells were set and independently observed in the FACS analyses. Because the compounds used in these experiments were equally cytotoxic to both populations of $\mathrm{T}$ cells in a dose-dependant manner, the populations were combined and treated as a single population of T cells. No direct immunosuppression was observed from pateamine or the contaminated peloruside as shown by the concomitant decrease in IFN- $\varepsilon$ and viability. Mycalamide, on the other hand, reduced proliferation and decreased IFN- $\varepsilon$ production without compromising cell viability, similar to the cyclosporin A control, suggesting that further research into the immunosuppressive activities of mycalamide are warranted.

\subsubsection{Effects of peloruside on Con-A stimulated splenocytes}

Since the new stock of peloruside had only been tested for cytotoxicity in a preliminary experiment (see Appendix B), it was cultured with splenocytes, alongside paclitaxel, to confirm its cytotoxicity. Peloruside was cytotoxic to proliferating splenocytes from Con-A stimulation with an $\mathrm{IC}_{50}$ value of $83 \mathrm{nM}$. Paclitaxel was also cytotoxic at an $\mathrm{IC}_{50}$ value of $30 \mathrm{nM}$. These $\mathrm{IC}_{50}$ values are 
comparable to what has been previously reported for peloruside $\left(\mathrm{IC}_{50}=1 \mathrm{nM}-66\right.$ nM; Hood, 2002; Gaitanos, 2005) and paclitaxel ( IC $_{50}=2$ nM - 53 nM; Gaitanos, 2005 ) in cancer cell lines in this laboratory. It is important to point out that Con A is a T cell mitogen, and splenocytes are typically comprised of $30 \%$ T cells, $60 \%$ B cells, and the remaining $10 \%$ are other leukocytes. As expected, complete ablation of MTT reduction did not occur in Con A-stimulated splenocytes treated with drugs at the higher concentrations (Figs. 3.2 b). Although, paclitaxel has been shown to be immunosuppressive on the basis of its cytotoxicity to proliferating B cells (Lee et al., 2000), there is conflicting evidence as to whether or not it is cytotoxic to proliferating T cells. In the studies by Lee et al.(2000), paclitaxel-induced cytotoxicity was not seen in Con A-stimulated splenocytes, contrary to what had been described previously (Brown et al., 1985). In the present study, the cytotoxicity of paclitaxel was confirmed in Con A-stimulated splenocytes with an $\mathrm{IC}_{50}$ value of $30 \mathrm{~nm}$. This value is in line with what others have seen using paclitaxel in cancer cell line cultures (Gaitanos, 2005; Gaitanos et al., 2004; Hood, 2002; Jordan et al., 1993). These studies and those of this thesis support the idea that anti-proliferative compounds may be of benefit to inflammatory autoimmune disorders by targeting the dividing populations of autoreactive T cells that mediate many of these diseases.

\subsubsection{Effects of peloruside in BMM $\emptyset$}

Peloruside, in contrast to paclitaxel, does not activate murine BMM $\varnothing$ to release the pro-inflammatory mediators TNF- $\varepsilon$, IL-12p40 and NO. The MTT reduction assay confirms that neither peloruside nor paclitaxel is cytotoxic to BMM $\varnothing$, and moreover, paclitaxel causes an increase in reductive metabolism in the 1 to $10 \mathrm{\varepsilon M}$ concentration range, possibly due to the induction of the pro-inflammatory 
machinery. When BMM $\varnothing$ were cultured with LPS and either drug at low $\varepsilon \mathrm{M}$ concentrations, there was a decrease in the production of TNF- $\varepsilon$ and NO, but not IL12p40. An interesting observation that followed from these results was that greater than $20 \mathrm{ng} / \mathrm{ml}$ LPS completely overshadowed the TNF- $\varepsilon$-reducing effects of paclitaxel and peloruside (data not shown). Taken together, these results indicate that unlike paclitaxel, peloruside does not induce the production of pro-inflammatory mediators and like paclitaxel, specifically reduces TNF-E and NO production by ВMM $\varnothing$ at lower concentrations of the drug.

Both paclitaxel and peloruside are known to be cytotoxic to proliferating cells by binding to a site on microtubules and preventing tubulin depolymerisation. Peloruside binds to a site on tubulin distinct from paclitaxel, although the microtubule stabilizing effect is similar (Gaitanos et al., 2004; Hood et al., 2002; Jimenez-Barbero et al., 2006; Pineda et al., 2004). Until now, however, there have been no investigations into the effects of peloruside on non-proliferating cells such as macrophages. The immunomodulatory effects and cytotoxic nature of paclitaxel, on the other hand, are well characterised. Its ability to cause apoptosis in dividing cells by binding to microtubules and preventing depolymerisation has made paclitaxel one of the most successful treatments for cancer to date (Gligorov and Lotz, 2004).

In addition to its tubulin-binding properties, paclitaxel also stimulates the murine TLR4 complex in a manner nearly indistinguishable from LPS (Byrd-Leifer et al., 2001). This stimulation occurs with concentrations of paclitaxel in the low $\varepsilon \mathrm{M}$ range, causing murine BMMØ to release inflammatory cytokines and NO. In these experiments we found TNF- $\varepsilon$ in BMM $\varnothing$ supernatants after $2 \mathrm{hr}$ but not $8 \mathrm{hr}$ exposure 
to paclitaxel. Other studies have reported higher levels of paclitaxel-induced TNF- $\varepsilon$ production for more prolonged periods (Bogdan and Ding, 1992; Byrd-Leifer et al., 2001). These discrepancies may be due to the use of thioglycolate to elicit peritoneal macrophages in the aforementioned studies (Bogdan and Ding, 1992; Byrd-Leifer et al., 2001), as thioglycolate is known to induce an inflammatory phenotype (Loke and Allison, 2003). The enhanced metabolic activity of BMM $\varnothing$ in response to paclitaxel has never been shown before, although it is not surprising that the energy requirements of an inflammatory response would increase the production of NADH and NADPH, thus enhancing MTT dye reduction (Berridge et al., 2005).

Since paclitaxel by itself induces an inflammatory response in BMMØ, it was important to determine if it synergises with LPS to enhance inflammation. Surprisingly, both paclitaxel and peloruside had no effect on the release of IL-12p40 from BMM $\varnothing$ cultured with LPS, but both drugs reduced the levels of TNF- $\varepsilon$ and NO in these LPS-stimulated BMM $\varnothing$ without being cytotoxic. The reduction of NO was significant, but not nearly as marked as the reduction of TNF- $\varepsilon$. The mechanism behind NO reduction may have to do with the reduction of TNF- $\varepsilon$, as the production of inducible nitric-oxide synthase (iNOS) - the enzyme primarily responsible for NO synthesis in MØ - is a downstream effect of TNF- (Vila-Del Sol et al., 2006). Although it is difficult to speculate at this time, one mechanism by which these compounds may specifically affect TNF-E production is through microtubulemediated secretion of TNF- $\varepsilon$ by BMM $\varnothing$ when stimulated by LPS. Previous studies have shown that the microtubule depolymerising compound, colchicine, inhibits macrophage production of TNF- $\varepsilon$, GM-CSF, and IL-6 in response to LPS (Rao et al., 1997). In contrast to the results presented here, no change was found in NO levels in 
the study by Roa et al. It is likely that the LPS response has a microtubule dependent and independent set of pathways. Similarly, microtubule stabilisation may be responsible for the decrease in LPS-induced production of TNF- $\varepsilon$ in response to peloruside or paclitaxel. Previous studies have established that TNF- $\varepsilon$ is a ratelimiting step in the pro-inflammatory process, and neutralisation of TNF- $\varepsilon$ by monoclonal antibodies causes a marked amelioration in several autoimmune diseases (Feldmann, 2002), as well as decreases paclitaxel-mediated NO production by peritoneal macrophages (Mullins et al., 1997). In addition, neutralisation of TNF- $\varepsilon$ also causes a reduction of GM-CSF and IL-6 (Feldmann et al., 1996) in a similar manner to what has been observed with microtubule-depolymerising compounds. The findings presented herein raise the possibility that selective microtubulestabilising compounds, such as peloruside, but not paclitaxel, may, in addition to having anti-mitotic effects on $\mathrm{T}$ cell population, be able to impact on inflammation as well by preventing secretion of TNF- $\varepsilon$ and the cascade of inflammatory mediators that follow. 


\section{Chapter 4: In Vivo Immunomodulatory Effects of}

\section{Paclitaxel}

\subsection{Introduction}

Paclitaxel is an extremely hydrophobic molecule. Because of this, it must be dissolved in solvents such as ethanol or DMSO. The clinically-used formulation of paclitaxel $\left(\right.$ Taxol $\left.^{\circledR}\right)$ is designed to overcome the challenges presented by the hydrophobicity of the compound. Taxol is comprised of paclitaxel dissolved in ethanol and Cremophor EL (1:1), diluted 5- to 20-fold in saline. Cremophor EL itself is not without effects, as it is known to cause hypersensitivity reactions (Price and Castells, 2002).

The LPS-mimicking inflammatory effects of paclitaxel, as shown in Chapter 3, are clearly demonstrated in vitro by others (Byrd-Leifer et al., 2001; Manthey et al., 1994; Perera et al., 1996). Surprisingly, however, there are few studies investigating paclitaxel-induced LPS-like toxicity in vivo. Mullins et al. (1998b) found that splenocytes from Taxol-injected mice showed a decreased proliferative response when isolated $24 \mathrm{hr}$ after injection. In addition, splenic macrophages isolated from these same mice showed enhanced NO production compared to untreated animals (Mullins et al., 1998a). There was no effect seen in control animals receiving Cremophor EL or saline. Taken together, these results suggest that Taxol exerts both anti-proliferative and inflammatory responses in vivo. 
As mentioned previously, one study looked at the use of a Cremophor EL-free micellar formulation of paclitaxel to treat Lewis rats immunised to develop EAE (Cao et al., 2000). This study found that paclitaxel was capable of decreasing the incidence and delaying the onset of EAE. With 3 treatment doses of $10 \mathrm{mg} / \mathrm{kg}$ bodyweight, only 3 out of 15 rats developed clinical disease, and these presented with a delayed onset and decreased severity when compared to the control group, which had $100 \%$ incidence. Interestingly, 11 of the 15 treated rats died for unexplained reasons only days after their final treatments. When they died, however, there were no EAE symptoms or spinal cord lesions present. There are no other published studies to date that look at the use of paclitaxel in EAE animal models of disease.

It should also be mentioned that bacterial LPS is a potent stimulator of inflammation, and exposure to LPS in the bloodstream can be fatal. Adverse effects of LPS toxicity include complement activation, fever, decreased cardiac output, and "leaky" vasculature in the lungs causing a fluid build-up. Ultimately, damage to the heart, lungs, and kidneys ensues, potentially leading to multiple organ failure. Interestingly, mice that lack a functional TLR4, such as $\mathrm{C} 3 \mathrm{H} / \mathrm{HeJ}$ mice, are resistant to the toxic effects of LPS (Sultzer, 1968).

\section{$\underline{\text { Aims }}$}

Because of adverse effects of LPS toxicity, the primary aim of this chapter was to determine whether the LPS-mimicry of paclitaxel, in combination with an enhanced inflammatory environment as is found in EAE, was responsible for the toxicity observed in the aforementioned study by Cao et al. (2000) in Lewis rats. Secondly, 
this chapter sought to determine if Taxol modified EAE in C57BL/6 mice using the same dosing regime established by Cao et al. (2000) in rats. The dosing regime used by Cao et al. (2000) involved three injections given on days 6, 8, and 12 after immunising for the induction of EAE. 


\subsection{Methods}

\subsubsection{Taxol preparation and administration}

Taxol, a formulation of paclitaxel dissolved in ethanol and mixed into Cremophor EL (1:1), was diluted between 5-fold and 10-fold in sterile PBS. Age- and sex-matched animals received Taxol $(10 \mathrm{mg} / \mathrm{kg}$ or $20 \mathrm{mg} / \mathrm{kg})$ in $500 \mathrm{\varepsilon l}$ injections i.p. on specified days.

\subsubsection{FACS staining and analysis}

Cells fixed in FACS buffer (Appendix A) were labeled with FITC-conjugated antiB220 antibody, PE-conjugated anti-CD8 antibody, and PerCP-conjugated anti-CD4 antibody (all from BD Pharmingen) to identify B cells, $\mathrm{CD} 8^{+} \mathrm{T}$ cells, and $\mathrm{CD} 4^{+} \mathrm{T}$ cells, respectively. Labeled cells were analysed on a FACScan (Chapter 2), and the proportions of specific cell subsets were determined with CellQuest Pro software (BD) (see Appendix C for gating).

\subsubsection{Re-stimulation of LN cells.}

Lymph nodes and spleens were removed from mice after the treatment regimes, and the splenocytes and LN cells were isolated as described in Chapter 2. Cells $\left(10^{6} /\right.$ well) were cultured with multiple concentrations of Con A, M.tb., or MOG for $24 \mathrm{hr}$ or 72 hr at $37^{\circ} \mathrm{C}$. Supernatants were removed for IFN- $\varepsilon$ detection by ELISA (see Chapter 3.2.5) and replaced with fresh CTCM, followed by the measurement of the percentage of cell proliferation by the MTT assay.

\subsubsection{Immunohistochemistry (IHC)}


Tissues, including spinal cord and intestine, were removed from mice and stored in zinc fixative (Appendix A). Paraffin-embedded sections ( $6 \varepsilon \mathrm{m}$ thick) were mounted on slides for IHC staining as described in Chapter 2 to determine if active proliferation had occurred. Sections were incubated with a 1:100 dilution of the marker antibody, anti-proliferating cell nuclear antigen (PCNA) (biotinylated mouse anti-vertebrate PCNA from PC10 clone; BD Pharmingen), and labeled cells identified by DAB staining. After counterstaining with methyl green, positive staining regions of tissue were identified by the distinctive brown DAB coloration under a light microscope. 


\subsection{Results}

\subsubsection{Mice survive the maximum tolerated dose of Taxol in an inflammatory environment.}

To determine if mice can survive treatments of Taxol in an inflammatory environment, wild-type mice or TLR4 -/- mice were immunised subcutaneously (s.c.) in the rear flanks with CFA (200 $\varepsilon 1$ IFA emulsified with $500 \varepsilon \mathrm{g} /$ mouse $M$. tuberculosis), and treated with $10 \mathrm{mg} / \mathrm{kg}$ Taxol, or the reported maximum tolerated dose (MTD) in C57BL/6 mice of $20 \mathrm{mg} / \mathrm{kg}$ of Taxol (Kim et al., 2001). This immunisation procedure is typically used to induce EAE, except these particular immunisations lacked the myelin antigen, which is obligatory for development of EAE. The Taxol treatment regime was the same as reported by Cao et al. (2000) [i.e. Taxol administration on days 6,8 , and 12 post-immunisation (p.i.)]. Because anaphylaxis and LPS toxicity are generally accompanied by laboured breathing and hyperthermia, respectively, general appearance, body temperatures, and body weights were monitored daily for the week of the Taxol treatments (see Chapter 2). Overall, body temperature was minimally affected by Taxol treatments in all of the treated groups (Fig. 4.1a). Moreover, no significant weight-loss occurred in any of the treatment groups (Fig. 4.1b). Blood serum from each group was tested for excessive inflammatory cytokines (IFN- $\varepsilon$; TNF- $\varepsilon$ ), but no differences were observed between groups (data not shown). Throughout these experiments, animals appeared healthy, active, and well-groomed. These data suggest that Taxol does not induce LPS-like toxic shock in wild-type mice following the treatment regime used by Cao et al. (2000). 

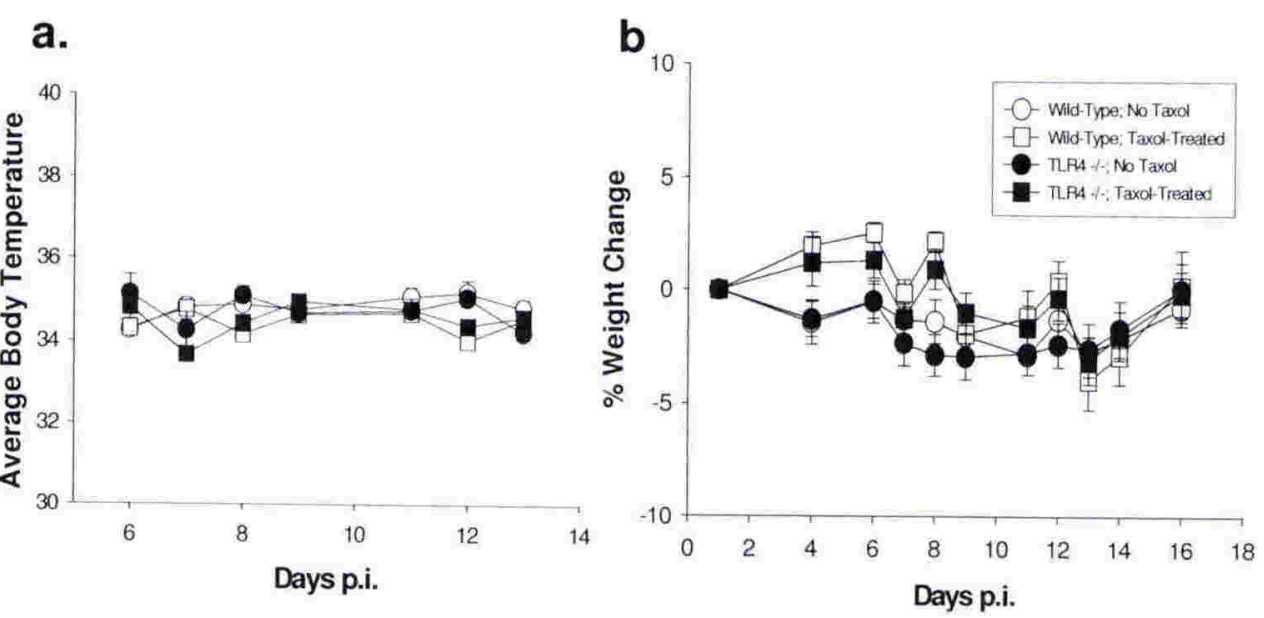

\section{Figure 4.1 Taxol has no effect on body temperature or weight.}

Wild-type (open) or TLR4 - - (filled) mice were treated with Taxol (squares) on days 6,8 , and 12, or were left untreated (diamonds). Weight (b) and body temperature (a) were measured daily for each treatment group. No significant differences were seen in body temperature or weight-loss between groups ( $n=3$ mice/group).

\subsubsection{Taxol on days 6, 8, and 12 p.i. does not affect EAE disease development.}

In the study by Cao et al. (2000) in rats, Taxol delayed the onset of EAE with treatments given on days 6,8 , and 12 p.i. These times correspond to the peak proliferation of peripheral myelin-reactive T cells, as well as the initial onset of symptoms (Juedes et al., 2000; Targoni et al., 2001). In the present studies in mice using doses of $10 \mathrm{mg} / \mathrm{kg}$ and the MTD of $20 \mathrm{mg} / \mathrm{kg}$, there was little difference between the disease incidence, severity, or day of onset between Taxol-treated and untreated animals (Fig. 4.2 a-b). When these data were combined with a previous experiment $(\mathrm{n}=5)$ using the same treatment regime and $10 \mathrm{mg} / \mathrm{kg}$ Taxol $(\mathrm{n}=$ 
10/group combined), there was still very little difference between disease onset and severity in Taxol treated animals versus controls (Fig. $4.2 \mathrm{c}$-d). Taken together, these data suggest that Taxol is ineffective at altering EAE symptoms and progression in C57BL/6 mice using this treatment regime.

To identify differences in lesions present within the spinal cord of the animals treated above, histological analysis was performed using hematoxylin and eosin (H\&E) stain on fixed tissue from animals harvested on day 20 p.i. (Fig. 4.3 a, c, \& e). In H\&E stained sections, lesions were seen as a dense accumulation of purple-stained inflammatory cell nuclei. The total number of lesions were determined for each spinal cord and individually given a score based on the size of the foci. To ensure that subjective scoring was equally applied to all spinal cords, the disease scores from each animal were not compared to the slide scores until after the slide scoring was completed. As typically seen in EAE animals, lesions were closely associated with disease severity - i.e., spinal cords with the most lesion scores of 2 or 3 came from the mice with the highest disease scores - regardless of whether or not they received Taxol (Fig. $4.3 \mathrm{a \& c}$ ). Exceptions, however, were inflammatory foci from animals in remission, which had fewer and milder inflammatory foci. Although the scores remained elevated in these remitting mice, it is likely that the damage incurred during the active disease was permanent, and further recovery is limited. Animals that did not show symptoms of EAE also did not have lesions in their spinal cord (Fig. 4.3 e). The total number of inflammatory foci in the Taxol treated group ( $34.8 \pm$ $11.6)$ and untreated group $(55.4 \pm 16.0)$ were not significantly different from one another (Student's $t$-test; $\mathrm{n}=5$ for both groups). 
a.

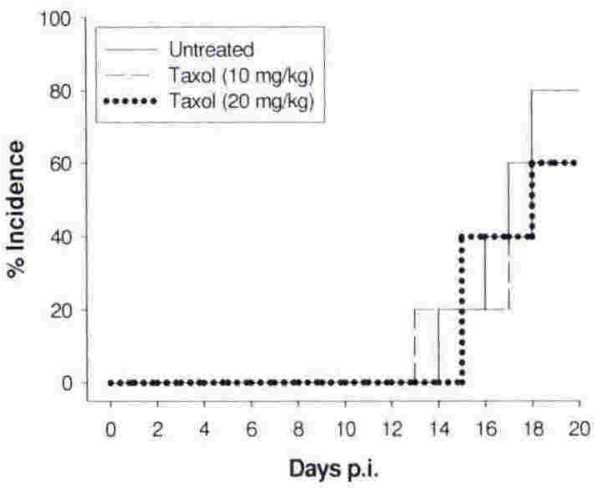

c.

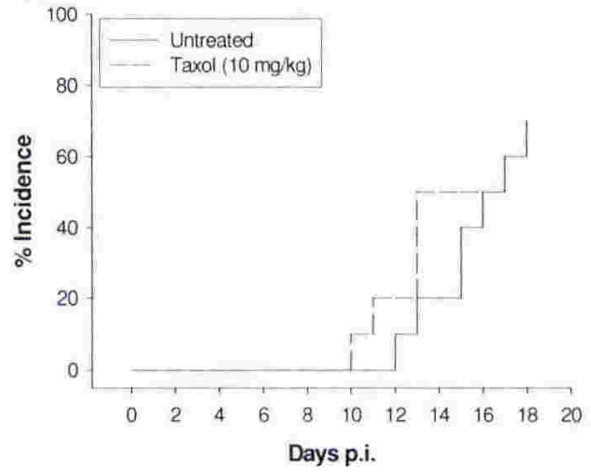

b.

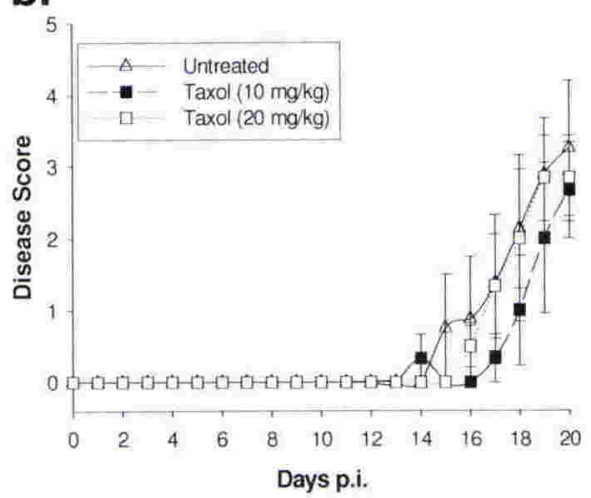

d.

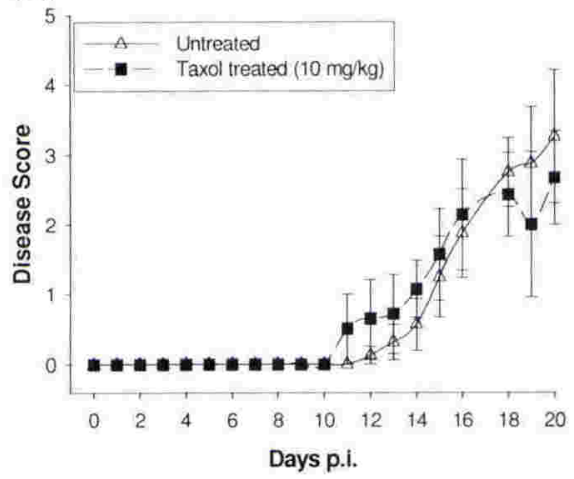

Figure 4.2 Taxol on days 6, 8, and 12 is ineffective at altering

EAE. Mice treated with Taxol at $10 \mathrm{mg} / \mathrm{kg}$ (dashed line; filled squares) or $20 \mathrm{mg} / \mathrm{kg}$ (dotted line; open squares) on days 6,8 , and $12 \mathrm{p.i}$. showed similar incidence $(a, c)$ and severity $(b, d)$ of EAE when compared to a control group (filled line; open triangles). $n=5$ (a-b); $\mathrm{n}=10$ including the 5 animals of $4.2 \mathrm{ab}(\mathrm{c}-\mathrm{d})$. 
To see if proliferation had occurred in the cellular infiltrates, IHC was performed on serially cut sections to label proliferating cells in S-phase in or near the lesions in the spinal cords. In these serially cut sections on which anti-PCNA IHC was performed, there was no evidence of proliferation within any of the lesions present in the spinal cords from Taxol treated and untreated animals (Fig. 4.3 b\&d, respectively). A portion of the intestine was used as a positive control for PCNA staining and, as expected, the enterocytes showed positive staining in the crypts, thus confirming that the cellular infiltrates were not proliferating within the spinal cord 20 days after immunisation, regardless of treatment status. 

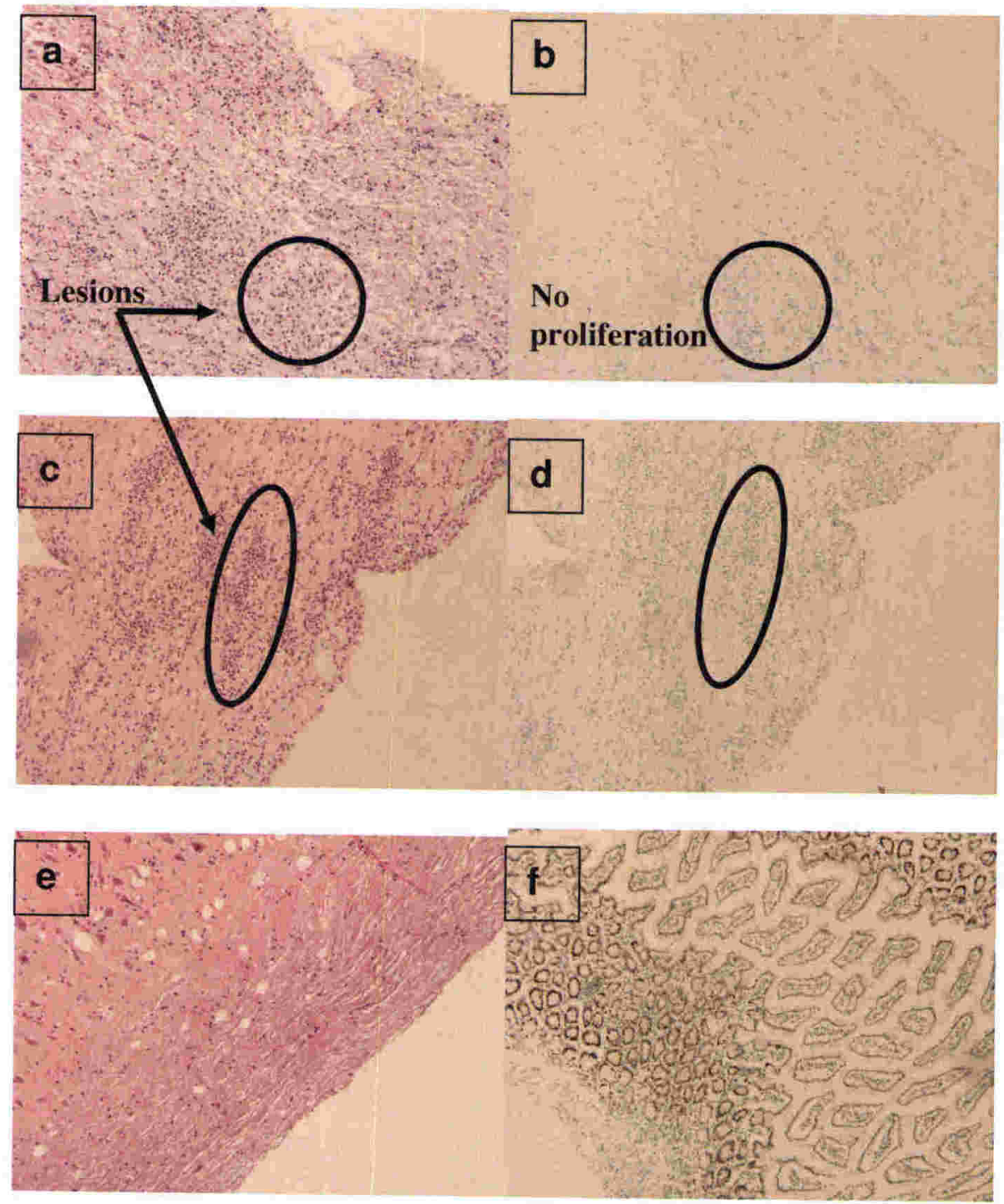

\section{Figure 4.3 EAE spinal cord lesions.}

Lesions are seen as a dense accumulation of H\&E stained cells in regions of myelin damage. Two representative lesion sites are circled, but other areas of lesions exist in the photomicrographs. The spinal cords of mice with EAE were stained with H\&E ( $a$ and $c$ ), or anti-PCNA using IHC (b and d). A portion of an intestine was used as a positive control for anti-PCNA staining (e); brown staining indicates the presence of PCNA, hence only the crypts, where proliferating enterocytes are expected to occur, are staining positive. No significant differences between the total number and severity of inflammatory foci were seen between Taxol-treated groups (a) and untreated groups (c) with EAE. In addition, the spinal cord infiltrates were not proliferating in any group at the time the tissue was harvested (day 20 p.i.). Shown here are representative pictures taken from 1 mouse/per group. Both mice shown were at peak disease $(4.0+)$ when tissues were harvested. Photographs were taken at x100 magnification. 


\subsubsection{Lymphocyte profiles are unaffected by Taxol treatments.}

In order to determine if selective depletion of specific cell populations in the lymph nodes was occurring following Taxol treatments, FACS analyses (see Appendix C) were performed on DLN cells isolated from the animals treated in the above investigations (Chapter 4.3.1 and 4.3.2) 20 days after the initial immunisations. In addition, two groups of mice immunised for EAE were included, a Taxol-treated and an untreated group. Lymph node cells were isolated and pooled within individual treatment groups. Because of pooling, the total number of $\mathrm{LN}$ cells from each treatment group was not known. Specific cell populations, including $\mathrm{CD} 4^{+} \mathrm{T}$ cells, $\mathrm{CD}^{+} \mathrm{T}$ cells, and $\mathrm{B} 220^{+} \mathrm{B}$ cells, were identified as a proportion of the total cells. Within the total populations of the LN from each pooled group, the proportions of $\mathrm{CD}^{+} \mathrm{T}$ cells, $\mathrm{CD} 8^{+} \mathrm{T}$ cells, and $\mathrm{B} 220^{+} \mathrm{B}$ cells remained largely unaffected in both wild-type and TLR4 -/- mice, regardless of whether or not the mice were immunised to induce EAE or treated with Taxol (Table 4.1), thus suggesting that Taxol does not preferentially alter specific lymphocyte subsets when administered in vivo. Whether or not Taxol, when administered with the current treatment regime, has an effect on the total number of LN cells remains unclear since quantitative collection of cells was not attempted. 
Injection d. 6, 8, and 12

\begin{tabular}{|c|c|c|c|c|}
\hline CD4+ & PBS & Cremophor EL & $\begin{array}{l}\text { paclitaxel } \\
(10 \mathrm{mg} / \mathrm{kg})\end{array}$ & $\begin{array}{l}\text { paclitaxel } \\
(20 \mathrm{mg} / \mathrm{kg})\end{array}$ \\
\hline \begin{tabular}{|l|} 
WT PBS \\
TLR4-/- PBS \\
\end{tabular} & $\begin{array}{l}27 \pm 1 \\
32 \\
\end{array}$ & $\begin{array}{l}27.5 \pm 1.5 \\
31\end{array}$ & $\begin{array}{l}29 \pm 7 \\
32.5 \pm 3.5\end{array}$ & $\begin{array}{l}23 \\
25 \\
\end{array}$ \\
\hline \begin{tabular}{|l|} 
WT CFA \\
TLR4-/- CFA \\
\end{tabular} & $\begin{array}{l}20 \pm 1 \\
24 \pm 0 \\
\end{array}$ & $\begin{array}{l}19.5 \pm 3.5 \\
23\end{array}$ & $\begin{array}{l}26 \pm 9 \\
26 \pm 6 \\
\end{array}$ & $\begin{array}{l}16 \\
19 \\
\end{array}$ \\
\hline WT EAE & 26 & - & 27 & E \\
\hline CD8+ & PBS & Cremophor EL & $\begin{array}{l}\text { paclitaxel } \\
(10 \mathrm{mg} / \mathrm{kg})\end{array}$ & $\begin{array}{l}\text { paclitaxel } \\
(20 \mathrm{mg} / \mathrm{kg})\end{array}$ \\
\hline \begin{tabular}{|l|} 
WT PBS \\
TLR4-/-PBS \\
\end{tabular} & $\begin{array}{l}28.5 \pm 2.5 \\
30\end{array}$ & \begin{tabular}{|l|}
$28 \pm 5$ \\
29 \\
\end{tabular} & $\begin{array}{l}29.5 \pm 0.5 \\
28.5 \pm 2.5\end{array}$ & $\begin{array}{l}27 \\
29 \\
\end{array}$ \\
\hline $\begin{array}{l}\text { WT CFA } \\
\text { TLR4- - CFA }\end{array}$ & $\begin{array}{l}18.5 \pm 2.5 \\
19.5 \pm 1.5\end{array}$ & $\begin{array}{l}17.5 \pm 2.5 \\
20\end{array}$ & $\begin{array}{l}21 \pm 5 \\
21 \pm 6 \\
\end{array}$ & $\begin{array}{l}16 \\
18 \\
\end{array}$ \\
\hline WT EAE & 21 & - & 19 & - \\
\hline $\mathrm{B} 220+$ & PBS & Cremophor EL & $\begin{array}{l}\text { paclitaxel } \\
(10 \mathrm{mg} / \mathrm{kg})\end{array}$ & $\begin{array}{l}\text { paclitaxel } \\
(20 \mathrm{mg} / \mathrm{kg})\end{array}$ \\
\hline \begin{tabular}{|l|} 
WT PBS \\
TLR4-/- PBS \\
\end{tabular} & $\begin{array}{l}32.5 \pm 1.5 \\
26 \\
\end{array}$ & $\begin{array}{l}36.5 \pm 4.5 \\
34\end{array}$ & $\begin{array}{l}32.5 \pm 6.5 \\
30 \pm 1\end{array}$ & $\begin{array}{l}40 \\
37 \\
\end{array}$ \\
\hline $\begin{array}{l}\text { WT CFA } \\
\text { TLR4- }- \text { - CFA } \\
\end{array}$ & \begin{tabular}{|l|}
$55.5 \pm 3.5$ \\
$50.5 \pm 1.5$ \\
\end{tabular} & $\begin{array}{l}58 \pm 8 \\
40 \\
\end{array}$ & $\begin{array}{l}48 \pm 15 \\
46.5 \pm 11.5\end{array}$ & $\begin{array}{l}61 \\
54 \\
\end{array}$ \\
\hline WT EAE & 46 & - & 44 & - \\
\hline
\end{tabular}

Table 4.1 Lymphocyte populations after Taxol treatments.

Lymphocytes were isolated from the draining lymph nodes on day 20 p.i. Wild-type (WT) or TLR4 -/- mice were immunized with PBS or CFA. Some WT mice also received CFA+MOG (EAE). On days 6, 8, and 12, animals received either PBS, Cremophor EL, Taxol (10 $\mathrm{mg} / \mathrm{kg}$ ), or Taxol $(20 \mathrm{mg} / \mathrm{kg})$ via i.p. injection. Cell population percentages were determined by FACS analysis and shown separately as CD4+ cells, CD8+ cells, and B220+ cells in descending order, respectively. Numbers without \pm error values indicate that the data were from a single experiment. All other data were from 2 experiments. 


\subsubsection{Re-stimulation of lymphocytes ex vivo causes inconsistent inflammation.}

Although lymphocyte populations were relatively unaffected by the Taxol

treatments, the proliferative or effector capacity of these lymphocytes may have been impaired. To test this, $\mathrm{LN}$ cells from mice that had undergone CFA or PBS

immunisations and the above treatments (Chapter 4.3.1 and 4.3.2) were re-stimulated with either M.tb. or Con A 20 days p.i. Levels of IFN- $\varepsilon$ and proliferation were determined for each group and compared. Three experiments were carried out, and the first and third experiments showed enhanced IFN- $\varepsilon$ production in cultures of LN cells from Taxol-treated WT animals; whereas, the second experiment showed decreased IFN- $\varepsilon$ production in all groups. Initial results showed that the LN cells from Taxol-treated WT mice produced higher levels of IFN-E in response to M.tb. (Fig. 4.4 a) compared to TLR4 -/- mice (Fig. 4.4 b), suggesting that Taxol enhances inflammation by a TLR4-dependent mechanism. A third experiment of a similar design, using LN cells from WT animals treated with $10 \mathrm{mg} / \mathrm{kg}$ or $20 \mathrm{mg} / \mathrm{kg}$ Taxol, also responded to M.tb. re-stimulations with enhanced IFN- $\varepsilon$ production (data not shown), lending support to the first experimental results. The second repeat of this experiment, however, failed to produce the same results. In this second experiment, the LN cells from Taxol-treated animals (WT and TLR4 -/-) produced lower levels of IFN- $\varepsilon$ in response to M.tb. (Fig. $4.4 \mathrm{c}-\mathrm{d}$ ) compared to the CFA-immunised/untreated controls. A similar result was obtained when these same LN cells from this second experiment were stimulated with Con A (Fig. 4.4 e-f). Moreover, a MTT assay performed on the cells from this second experiment showed that extensive proliferation occurred and to a similar level in all groups in response to Con A (Fig. $4.4 \mathrm{~g}-\mathrm{h}$ ), indicating that the lack of IFN- $-\varepsilon$ production was not due to an inhibition of proliferation. Taken together, the data obtained from the second experiment suggest 
that Taxol interferes with IFN-E production in an unknown, TLR4-independent manner. Because of the pooled samples and timing of LN removal in all three experiments, it is not clear if the observed differences were related to the Taxol treatments, stage of immune response to the immunisation, or simply experimental variation. To clarify this, the experimental design would have to be modified to eliminate these variables. 
a.

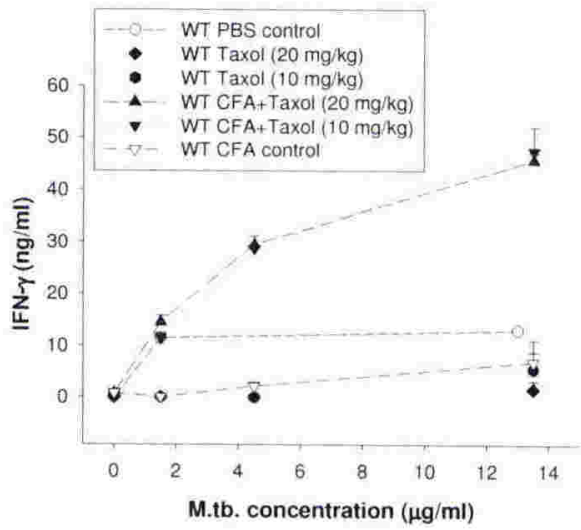

c.

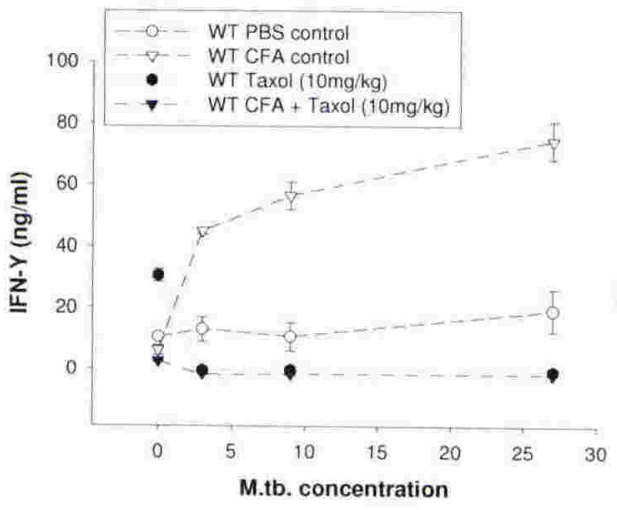

e.

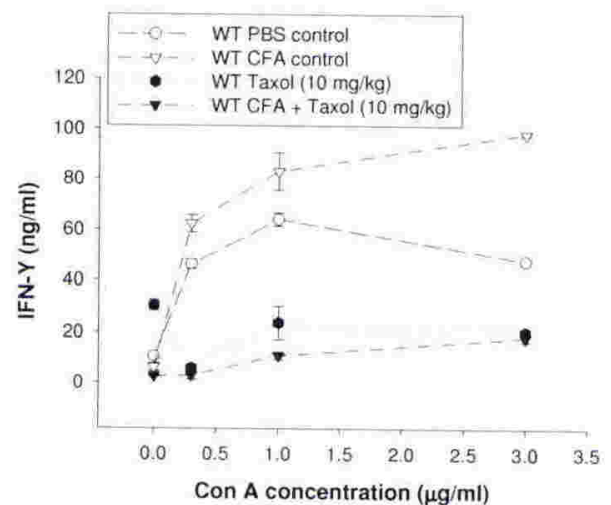

b.

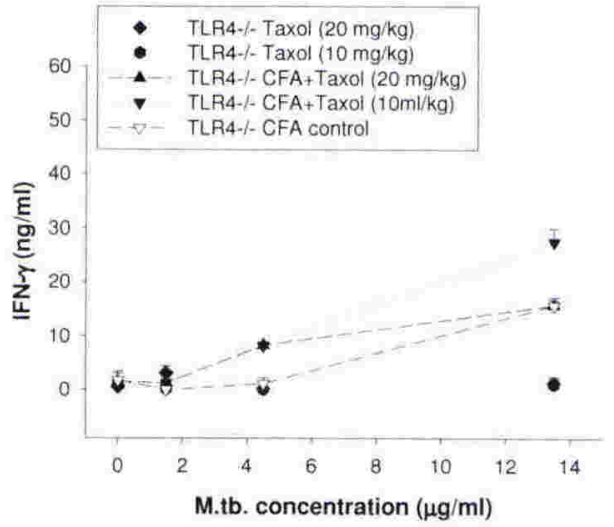

d.

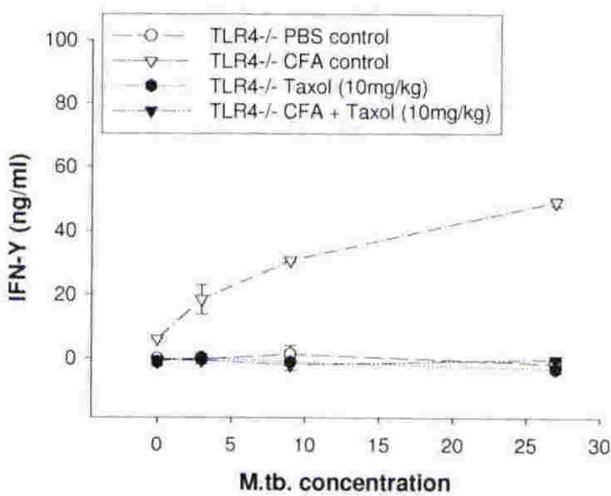

f.

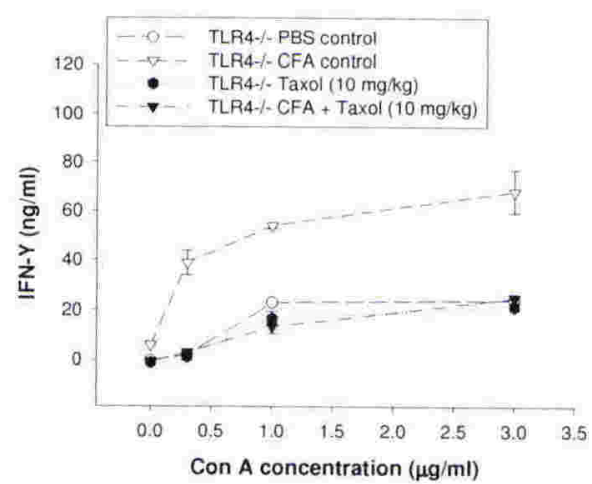


g.

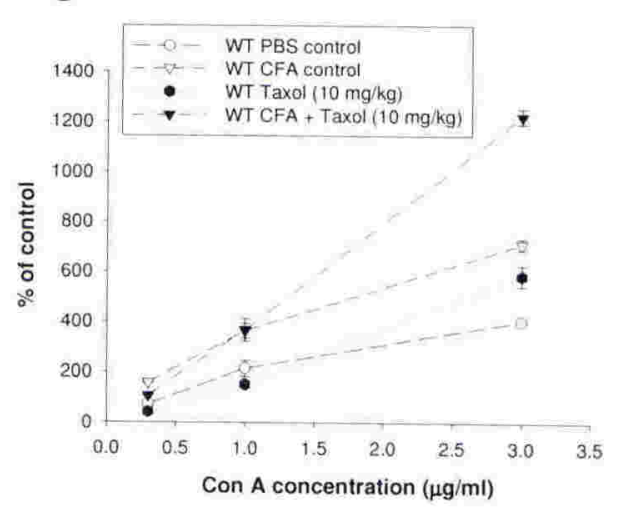

h.

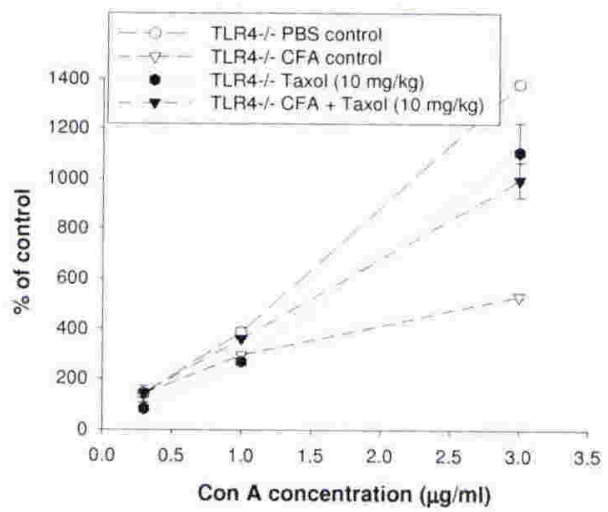

\section{Figure 4.4 Lymphocyte re-stimulations.}

Draining lymph node cells were isolated from WT (a, c, d, and e) and TLR4 - / (b, d, f, and g) mice that had been immunised with CFA (triangles) or nothing (all other shapes), and were then treated with PBS (open shapes), or Taxol (filled shapes) on days 6,8 , and 12 p.i. Cells were cultured for $72 \mathrm{hr}$ with M.tb. (a-d) or Con A (e-h). Supernatants were removed for IFN- $\leq$ detection ( $a-f$ ), or assayed for proliferation by MTT (gh). Three experiments were performed. Results from Exp 1 are presented in panels a-b. Exp 2 results are given in panels $c-h$. Results from Exp 3 (data not presented) were similar to Exp 1. Panels e \& f show that Con Astimulated cells respond similarly to the M.tb.-stimulated cells from c \& d. The MTT assay $(\mathrm{g}-\mathrm{h})$ shows that the lack of IFN- $\leq$ production (e-f) was not due to inhibition of proliferation. 


\subsubsection{A single injection of Taxol has no impact on LN cell, splenocyte, or intestinal cell proliferation in vivo.}

A previous study found that Taxol $(15-30 \mathrm{mg} / \mathrm{kg})$ administered in vivo inhibits proliferation in Con A-stimulated splenocytes and purified $\mathrm{CD} 4^{+} \mathrm{T}$ cells ex vivo after $24 \mathrm{hr}$ (Mullins et al., 1998b). In the present study, to determine the recovery time of lymphocytes after a single treatment of Taxol, splenocytes and LN cells were isolated 1 or 6 days after a single injection of Taxol $(20 \mathrm{mg} / \mathrm{kg})$, followed by Con A re-stimulation ex vivo for 24 or $72 \mathrm{hr}$. The 72 -hr culture was performed to ensure that cells have had the full opportunity to proliferate, since $24 \mathrm{hr}$ might not have been enough time for sufficient proliferation to occur. In contrast to the previous report (Mullins et al., 1998b), there was little to no difference between the proliferative capacity of either splenocytes or LN cells from Taxol-treated animals compared to untreated animals at any time following the initial injection (Fig. 4.5). The 24-hr cultures (Fig. 4.5 a-b) are expressed as a percentage of the unstimulated control to show that cells do, in fact, proliferate in response to Con A within this short time period, despite the earlier Taxol treatment. Because unstimulated splenocytes and LN cells do not survive very long in culture, data from cells stimulated for $72 \mathrm{hr}$ (Fig. 4.5 c-d) are shown as raw absorbance values, rather than a percentage of the unstimulated controls. Although there does appear to be slight differences in the degree of proliferation of lymph node cells after $72 \mathrm{hr}$ (Fig. $4.5 \mathrm{c}$ ), this difference is not significant with $3 \varepsilon \mathrm{g} / \mathrm{ml}$ Con A stimulation, and is thus likely to be an artifact. Also, this difference is not consistent with the splenocyte data, nor the 24-hr lymph node cultures. In addition, this experiment was repeated with a lower dose of Taxol $(10 \mathrm{mg} / \mathrm{kg}$ ) with similar results (data not shown). These data suggest that a transitory effect of a single Taxol treatment is not present in naïve T cells after $24 \mathrm{hr}$. 


\section{Lymph node cells}

a.
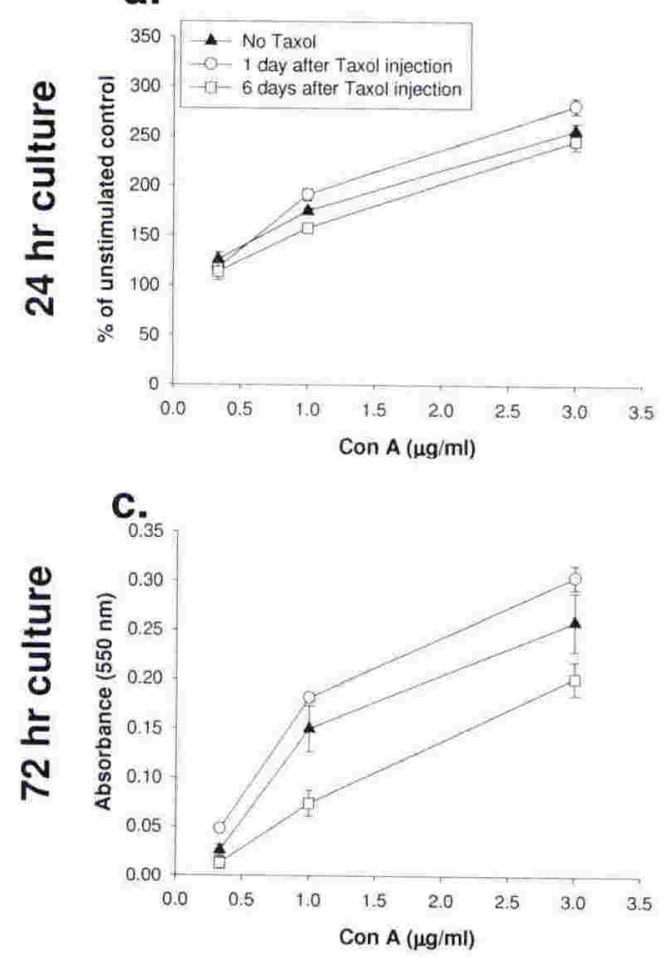

Splenocytes

b.

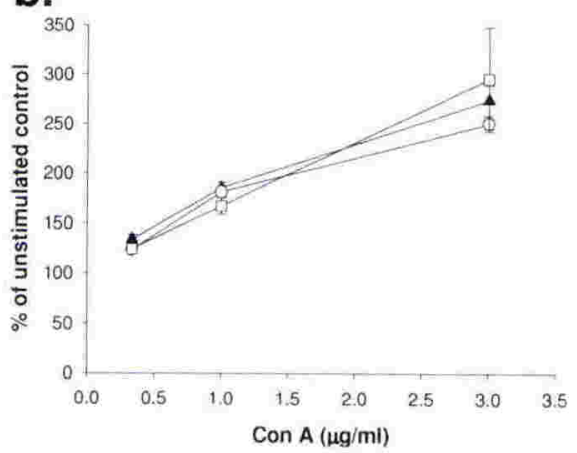

d.

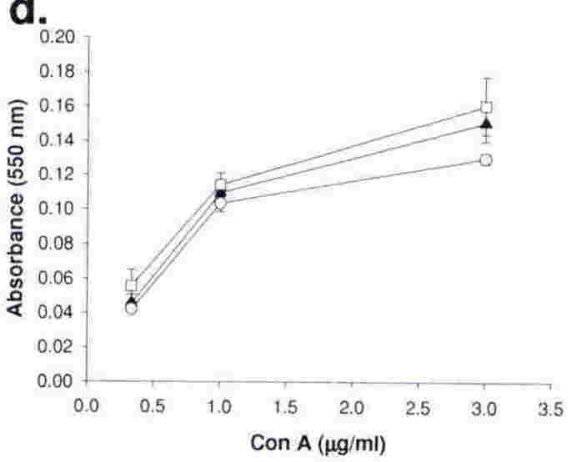

Figure 4.5 A single Taxol treatment has no effect on proliferation. Taxol (20 mg/kg) was administered to mice either 1 day (open circles) or 6 days (open squares) before removing lymph node cells or splenocytes. Cells were cultured with three Con A concentrations for $24 \mathrm{hr}(\mathrm{a} \& \mathrm{~b})$ or 72 hr (c\&d). Proliferation was determined by the MTT assay. Cells from Taxol-treated mice showed little difference in proliferation when compared to cells from untreated mice (closed triangles). A Student's $t$ test showed no significant difference between Taxol-treated cells and the untreated cells cultured with $3 \mathrm{gg} / \mathrm{ml}$ Con A.

In a similar experiment, animals were treated with $10 \mathrm{mg} / \mathrm{kg}$ Taxol, followed by the removal of a portion of intestine at $1,4,7$, or 13 days after the Taxol injection. The intestinal portions were fixed, paraffin-embedded, and mounted on slides. IHC analysis with an anti-PCNA antibody was performed to determine if a single 
injection of Taxol could inhibit proliferation from constantly dividing cells, such as those in the intestinal crypts. Similar to what was found in LN cells and splenocytes with a single injection of Taxol, there was no difference in intestinal cell proliferation between any of the animals tested at all time points (Fig. 4.6). This is evident by the brown staining in the intestinal crypts, where proliferation constitutively occurs, in all samples. The negative IHC-staining control sample shows no background staining occurred in the intestinal crypts (Fig. $4.6 \mathrm{f}$ ). The potency of the paclitaxel stock solution used in these experiments was confirmed in other experiments carried out at about the same time, as well as separate experiments performed simultaneously by other members of this laboratory in their own studies. It was concluded therefore that either the dose of Taxol is insufficient to inhibit intestinal cell proliferation, or that a single dose of Taxol is not enough to inhibit proliferation in vivo. 
a.

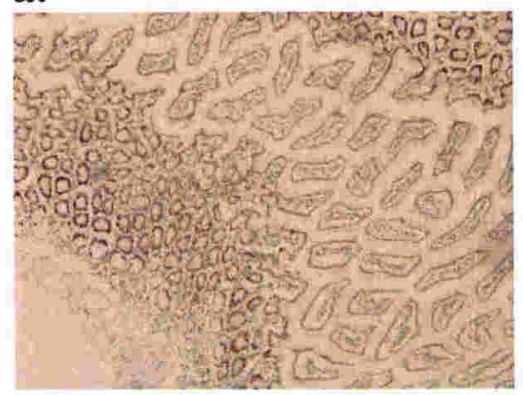

c.

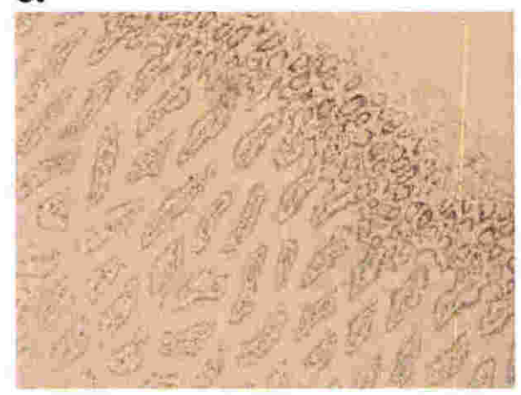

e.

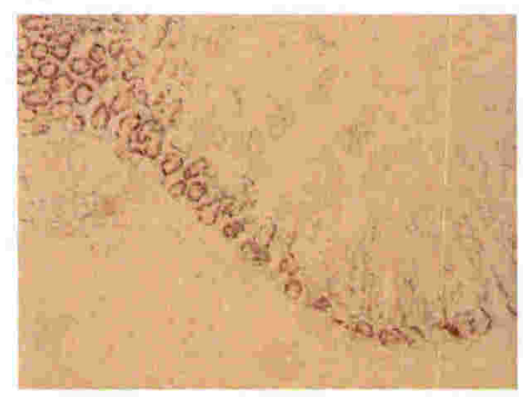

b.

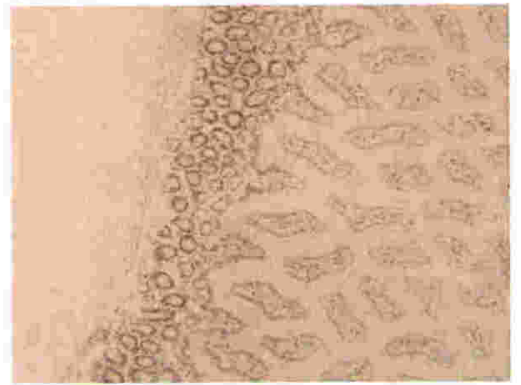

d.

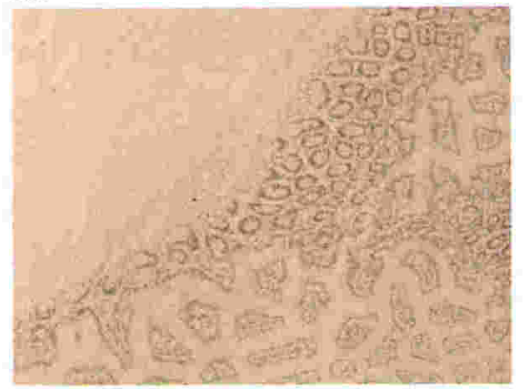

f.

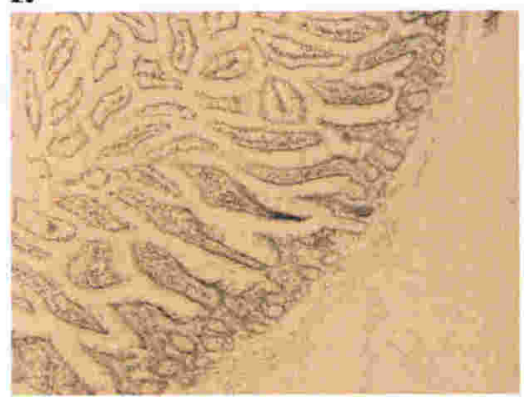

Figure 4.6 Proliferation occurs in intestines after single injection of

Taxol. A single injection of Taxol $(10 \mathrm{mg} / \mathrm{kg})$ was administered to mice, and the intestines were removed following 1 day (a), 4 days (b), 7 days (c), or 13 days (d) to identify transitory effects of Taxol. An untreated mouse intestine (e) was used as a positive control. The negative control ( $f$ ) was obtained by not using the anti-PCNA antibody during IHC staining. Enterocyte proliferation occurred in all samples, evident by the brown-stained cells in the intestinal crypts. Pictures were taken under x100 magnification. 


\subsection{Discussion}

Taxol induces the production of inflammatory cytokines from murine macrophages in vitro at concentrations above $1 \varepsilon \mathrm{M}$. Based on the total body volume of a mouse, these concentrations are likely to be surpassed when Taxol is injected i.p. at the concentrations used in the experiments above; therefore, previously reported paclitaxel toxicity could have been an inflammatory-associated event. Although localised Taxol concentrations in these experiments were not tested in specific tissues (blood, fat, peritoneal cavity, etc.), human studies that looked at i.p. injections found that a rapid uptake (within hours) and sustained bioavailability occurs when paclitaxel is administered i.p. (Gelderblom et al., 2002). Taxol was injected into mice that were immunised with an inflammation-causing adjuvant, CFA. In this study, however, these animals tolerated the dosing regime chosen from a previous study in rats by Cao et al. (2000). Even at the MTD, the animals appeared healthy and were visibly and physically unaffected. To repeat previous published findings regarding the impact of Taxol on EAE (Cao et al., 2000), the same dosing regime was tested in mice receiving EAE immunisations. There was no difference in disease onset or severity between control mice and Taxol-treated mice. Moreover, lymphocyte profiles were very similar between the two groups one week after Taxol treatments. There was not a consistent alteration in inflammation when these lymphocytes were restimulated. Furthermore, the proliferative capacity of lymphocytes that were isolated from Taxol-treated mice was unaffected as little as $24 \mathrm{hr}$ after the Taxol treatment. Thus, it was concluded that Taxol, with the reported treatment regimes, does not noticeably enhance inflammation, nor does it affect EAE disease progression in C57BL/6 mice. 
The previous study by Cao et al. (2000) found paclitaxel, delivered in a micelle formulation, delayed the onset of EAE in Lewis rats, but the authors failed to comment on the LPS-mimetic properties of paclitaxel. The fact that 11/15 animals treated with what was determined to be the best disease-delaying concentration of paclitaxel succumbed to an unidentified toxic effect was unfortunate, especially since paclitaxel is a very inexpensive and readily available anti-cancer compound. In the present study, to determine if Taxol displayed inflammatory properties in vivo, the drug was administered to WT mice and TLR4 -/- mice and the effects compared to identify potential toxicities. Because paclitaxel does not induce inflammation in TLR4 -/- murine macrophages, TLR4 -/- mice were assumed to be good controls since no TLR4-mediated inflammation should be present. Surprisingly, different doses of Taxol did not have any visible effect on either group, nor was there a significant difference in weight or body temperature. The lack of a pyrogenic response to Taxol in wild-type animals was particularly surprising due to the TLR4stimulating nature of the drug. Mice that lack TLR4 are resistant to the pyrogenic effects of LPS, indicating that TLR4-stimulation is crucial for hyperthermia. It is unclear why Taxol did not induce a pyrogenic response, since both LPS and paclitaxel stimulate TLR4 in a comparable manner.

Since the MTD of Taxol was shown to be safely tolerated in C57BL/6 mice (discussed above), mice were immunised with MOG/CFA to induce EAE, and were then treated with Taxol on days 6,8 , and 12 p.i. For unknown reasons this Taxoldosing regime did not have the same effects in the C57BL/6 mouse model of EAE as previously reported in a Lewis rat EAE model (Cao et al., 2000), although 
differences in response between mice and rats is not unexpected, since even the species of mouse may affect EAE induction profiles.

Macrophages in C57BL/6 mice have shown enhanced production of inflammatory mediators 24 hr ex vivo after treatments with Taxol (Mullins et al., 1998a). Here, an attempt was made to determine if Taxol treatments enhanced the inflammatory capacity of LN cells isolated from immunised, Taxol-treated C57BL/6 mice. Initial results suggested that there may have been a TLR4-dependent enhancement of inflammation in M.tb.-restimulated LN cells from Taxol-treated animals; however, this was only seen in two out of three experiments. In the second, negative experiment, LN cells from Taxol-treated animals responded differently to the controls, in that IFN-E levels were reduced by a TLR4-independent mechanism. This is interesting, because in all three experiments, the DLN cells isolated from the Taxol-treated mice were responding differently to the controls, but in dissimilar ways. It is possible that the timing of the re-stimulations affected the outcome, since they were performed approximately, but not exactly, one week after the final treatment of Taxol. In addition, the LN cells from each treatment group were pooled, introducing the possibility that an individual mouse skewed the results of the group. Experimental variation cannot be ruled out either, since the immunisations in each experiment may have minor differences that affect the immunopathology, including the timing of inflammatory resolution in each experiment. While these results suggest that paclitaxel has the ability to enhance inflammation in vivo, care must be taken in interpreting these results due to the variability between mice and their in vivo inflammatory responses to drugs. 
The increased production of IFN- $\varepsilon$ in DLN cultures from Taxol-treated WT mice, but not TLR4 - $/$ - mice, suggests that Taxol has affected the immune response through LPS mimicry. This would also indicate that the effects of Taxol lasted more than one week, since the LN cells were not harvested until one week after the last Taxol treatment. To see if this effect of Taxol on enhanced IFN- $\varepsilon$ production was genuine, a new experimental design would be needed, such as harvesting DLN cells $24 \mathrm{hr}$ after the last Taxol treatment, to reduce the variables mentioned above.

Re-stimulations of purified T cells with Con A have shown that Taxol exerts antiproliferative effects ex vivo $24 \mathrm{hr}$ after injecting the mice from which the T cells were obtained (Mullins et al., 1998b). These findings, however, are in contrast to the data presented in Fig. 4.5. Not only was a single dose of Taxol ineffective at inhibiting LN cell proliferation $24 \mathrm{hr}$ later, but splenocytes were also unaffected, despite using two different ex vivo culturing times ( $24 \mathrm{hr}$ and $72 \mathrm{hr}$ ). In addition, intestines were removed from the Taxol-treated animals to see if the enterocyte proliferation in the crypts was affected. Intestinal crypts are constitutively proliferating; therefore, they are likely to be sensitive to an anti-mitotic agent like Taxol. The intestinal crypts showed proliferation was still occurring at all time-points tested in both the control and Taxol-treated animals. The potency of the Taxol used in these experiments was demonstrated in Chapter 3, ruling out the possibility that the Taxol stock solution had lost its potency. Taken together, this evidence is highly suggestive that a single dose of Taxol is unable to affect $\mathrm{T}$ cell proliferation, or proliferation of other non-immune cells in vivo. 
One possible problem with treatments spaced over $24 \mathrm{hr}$ apart, such as the treatments performed in this study $(6,8$, and 12 days), is that a prolonged time period may allow naïve $\mathrm{T}$ cells to respond to the immunising antigen. The MOG/CFA emulsion creates a subcutaneous cyst with persistent antigen exposure, providing the DLN cells with a constant source of MOG. As shown in Fig. 4.5, naïve lymphocytes were unaffected by the anti-proliferative effects of Taxol after at least $24 \mathrm{hr}$ following treatment. The cytotoxicity of Taxol has been shown to be a function of the time to drug exposure over a certain threshold concentration (Helson et al., 1993). This threshold concentration may be breached using a widely-spaced treatment regime, allowing naïve MOG-reactive T cells to become activated and proliferate during the treatment windows between days 6 and 8 , or days 8 and 12. Since clonal expansion occurs very quickly, it is possible that in the days between Taxol treatments these expanded $\mathrm{T}$ cells stop expanding, avoiding the cytotoxic effects of Taxol, and migrate into the periphery where they can eventually become encephalogenic. It may be necessary to close these "windows" of proliferation in order to see an effect in EAE by giving more closely spaced injections of Taxol.

Overall, these experiments suggest that Taxol does not induce a toxic inflammatory response in mice, which could have explained the high lethality observed by Cao et al. (2000) in rats. Also, the results suggest that the Taxol dosing regime performed here, as used in the rat model by Cao et al. (2000), may not be suitable for impacting on EAE progression in mice. Interestingly, paclitaxel is a substrate for the P-gp efflux pump, which is known to be active in many drug-resistant cancer cells, as well as organ-specific tissue types (Maher et al., 2005; Richaud-Patin et al., 2004). The Pgp efflux pump acts by removing selective drugs of broad substrate characteristics, 
including paclitaxel, from the cell cytoplasm causing the cell to be resistant to what is typically a lethal dose of the drug in the absence of overexpression of the pump. Intestinal and $\mathrm{T}$ cells are known to express high levels of P-gp on their membrane surfaces (Elliott et al., 2004; Maher et al., 2005), and this multi-drug resistance effect might in part explain why a single dose is ineffective. In Chapter 3, paclitaxel was found to be cytotoxic to proliferating splenocytes, but this is likely due to the different effects of P-gp in vitro and in vivo (Fig. 4.7). One way to circumvent the effects of the P-gp pump would be to inhibit the pump with an inhibitor such as verapamil. Another way may be to override the action of the efflux pump by maintaining a higher blood concentration and a more prolonged exposure to paclitaxel by treating mice daily for a fixed number of days shortly after immunisation. It is likely that Taxol may still modify EAE disease progression in C57BL/6 mice, but that the Taxol dosing regime may need to be modified to optimise its effects in vivo. Chapter 5 confirms that this is indeed the case by demonstrating a clear effect of Taxol on EAE disease progression using a modified treatment regime. 



\section{Chapter 5: Effects of Anti-Mitotic Drugs on EAE}

\subsection{Introduction}

Taxol is widely used in murine models of cancer, and while it has inflammatory properties in vitro, there is very little evidence of these inflammatory aspects affecting Taxol treatments in murine cancer studies. As discussed in the previous chapter, the clear inflammatory effects of Taxol in vitro may not be easily detectable in live animals. The investigative approach to identify inflammation in the previous chapter could have been modified by adjusting the time of the assays to take place more closely to the time immediately following Taxol administrations. There is also the possibility that there were no inflammatory effects to detect due to regulatory or compensatory mechanisms, such as secretion of anti-inflammatory cytokines, which could dampen down the inflammatory effects. For these reasons, Taxol still holds promise as a treatment for EAE due to the anti-mitotic nature of the drug and limited known side-effects in vivo.

Because previous efforts to treat EAE with paclitaxel relied on markedly spaced treatments, a tighter dosing regime may be needed to circumvent the proliferative "windows" of the T cells. This tighter regime may also partially overcome the possible P-gp clearance of Taxol from the T cells by maintaining a higher average plasma concentration of the drug. When injected intravenously, Taxol has a half-life of only $20 \mathrm{~min}$ in the serum of mice (Kim et al., 2001). Evidence in humans suggests that this half-life can be prolonged with i.p. administration of Taxol (i.v. 17.0 \pm 11.3 h versus i.p. $28.7 \pm 8.72 \mathrm{~h}$; Gelderblom et al., 2002), but if this is true in mice then the relative half-life of i.p. Taxol treatments would be measured by hours, not days. 
Because Taxol is cleared quickly in mice, windows of T cell proliferation could easily occur between treatment doses if the doses are given greater than $24 \mathrm{hr}$ apart. Therefore, by treating animals daily for 5 days, serum paclitaxel concentrations will remain elevated for longer, allowing effective concentrations of paclitaxel to persist in and around the proliferating $\mathrm{T}$ cell targets.

Peloruside, like paclitaxel, is a microtubule-stabilising compound that has similar anti-mitotic activity to paclitaxel, but it also has a distinct, non-taxoid binding site on tubulin (Gaitanos et al., 2004; Jimenez-Barbero et al., 2006; Pineda et al., 2004). In Chapter 3, it was shown that peloruside did not mimic LPS in the same fashion as Taxol and, moreover, it is reported to be less of a substrate for the P-gp efflux pump (Gaitanos et al., 2004). This means that peloruside, in contrast to Taxol, may kill proliferating cells without causing TLR4-mediated inflammation. In addition, because it is less of a substrate for the P-gp pump, concentrations of peloruside may remain elevated within cells that express P-gp mediated multi-drug resistance, such as T cells (Richaud-Patin et al., 2004).

\section{$\underline{\operatorname{Aims}}$}

The aim of the studies in this Chapter were to test whether a more intensive Taxol treatment regime, as used in murine cancer models, can modify EAE disease progression. Furthermore, the efficacy of peloruside, an anti-mitotic drug that lacks the LPS mimicry of paclitaxel, would also be tested as an alternative to paclitaxel for the treatment of EAE. Because the supply of peloruside was very limited, especially for in vivo testing, it was expected from the outset that only a few critical in vivo 
investigations with peloruside could be attempted, and that the bulk of the experiments in this chapter would be carried out with Taxol.

\subsection{Methods}

\subsubsection{Drug preparations}

A pre-formulation of Taxol (Bristol-Myers Squib; $100 \mathrm{mg} / 16.7 \mathrm{ml}$ of ethanol/Cremophor EL) was diluted 5-10 fold in sterile PBS before being administered i.p. to mice at a dose of $10 \mathrm{mg} / \mathrm{kg}$ or $20 \mathrm{mg} / \mathrm{kg}$. Purified peloruside was dissolved in $100 \%$ ethanol and then diluted 10-fold in PBS prior to i.p. administration at a dose of $10 \mathrm{mg} / \mathrm{kg}$. This dose of peloruside was chosen because previous studies determined that $10 \mathrm{mg} / \mathrm{kg}$ was effective at treating cancer in mice (Peter Northcote; personal communication).

\subsubsection{FACS analysis of DLN cells}

Cells were isolated from the DLNs of C57BL/6 wild-type mice as described in Chapter 2. These cells were then incubated with anti-CD4-PerCP, anti-CD8-PE, and anti-CD25-FITC antibodies to determine the percentage of these specific cell types that were present within the DLNs. Cell events were counted using a FACS analyser, and data were analysed using CellQuest software.

\subsubsection{Assessment of in vivo proliferation}

LN cells and splenocytes were isolated from 2D2 mice with a C57BL/6 background, which are transgenically modified such that the majority of $\mathrm{CD} 4^{+} \mathrm{T}$ cells express a TCR specific for the $\mathrm{MOG}_{35-55}$ peptide (Bettelli et al., 2003). The isolated cells were 
stained with the cytoplasmic dye 5, 6-carboxyfluorescein diacetate succinimidyl ester (CFSE; Sigma) by incubating the cells in $63 \mathrm{ng} / \mathrm{ml} \mathrm{CFSE} \mathrm{in} \mathrm{PBS} \mathrm{for} 8 \mathrm{~min}$, followed by addition of FCS to stop the reaction. After washing the cells in PBS, $2 \times 10^{7}$ cells were transferred to each normal C57BL/6 mouse 1 day prior to immunisation with MOG/CFA. After 4-5 days, LN cells from Taxol-treated or untreated mice were isolated and analysed by flow cytometry. 


\subsection{Results}

\subsubsection{Taxol treatments of $10 \mathrm{mg} / \mathrm{kg}$ are insufficient to significantly alter EAE}

progression.

Concentrations of Taxol used to treat cancer in mice vary, but $10-20 \mathrm{mg} / \mathrm{kg}$ of bodyweight is a commonly administered dose range (Kim et al., 2001; Zhang and Russell, 2006). A previous study injected $10 \mathrm{mg} / \mathrm{kg}$ Taxol i.p. daily for 10 days without any obvious toxicity in mice (Yuan et al., 2000), providing the basis for a new dosing strategy in the present study. The dose of Taxol $(10 \mathrm{mg} / \mathrm{kg})$ was chosen because of previous results in Chapter 4 that showed that $10 \mathrm{mg} / \mathrm{kg}$ of Taxol gave a similar, if not slightly more effective response than $20 \mathrm{mg} / \mathrm{kg}$ of modifying inflammation in vivo. Taxol $(10 \mathrm{mg} / \mathrm{kg})$ was administered daily for 5 days to mice pre-immunised with MOG/CFA. The 5 daily treatments were administered over one of three time periods from days a) $1-5$, b) 5-9, or c) $10-14$ p.i. These time periods were chosen because they encompass key cellular events that lead to the development of EAE.

Disease incidence was uncharacteristically low in this particular set of animals (40\% in the control group, $50 \%$ overall). Usually, $80-100 \%$ of animals immunised develop some form of the disease. The median day of initial onset for mice that developed EAE from all groups was also unusually late (day 19). Symptoms typically appear around day 12. Disease severity of the sick animals was similar among all three groups given Taxol, as well as the untreated group. Upon close analysis of the sick animals in each group, however, a slight difference in the timing of disease onset was apparent (Fig. 5.1), suggesting that, in fact, 5 daily doses of Taxol may have impacted on EAE, delaying the development but not altering the severity of the 
disease. A more important observation was the fact that none of the animals died from any of the three treatment regimes, indicating that toxicity is not an issue with 5 daily injections of Taxol given at a dose of $10 \mathrm{mg} / \mathrm{kg}$. Unfortunately, significant differences of onset could not be determined due to low numbers of sick control animals $(\mathrm{n}=2)$.

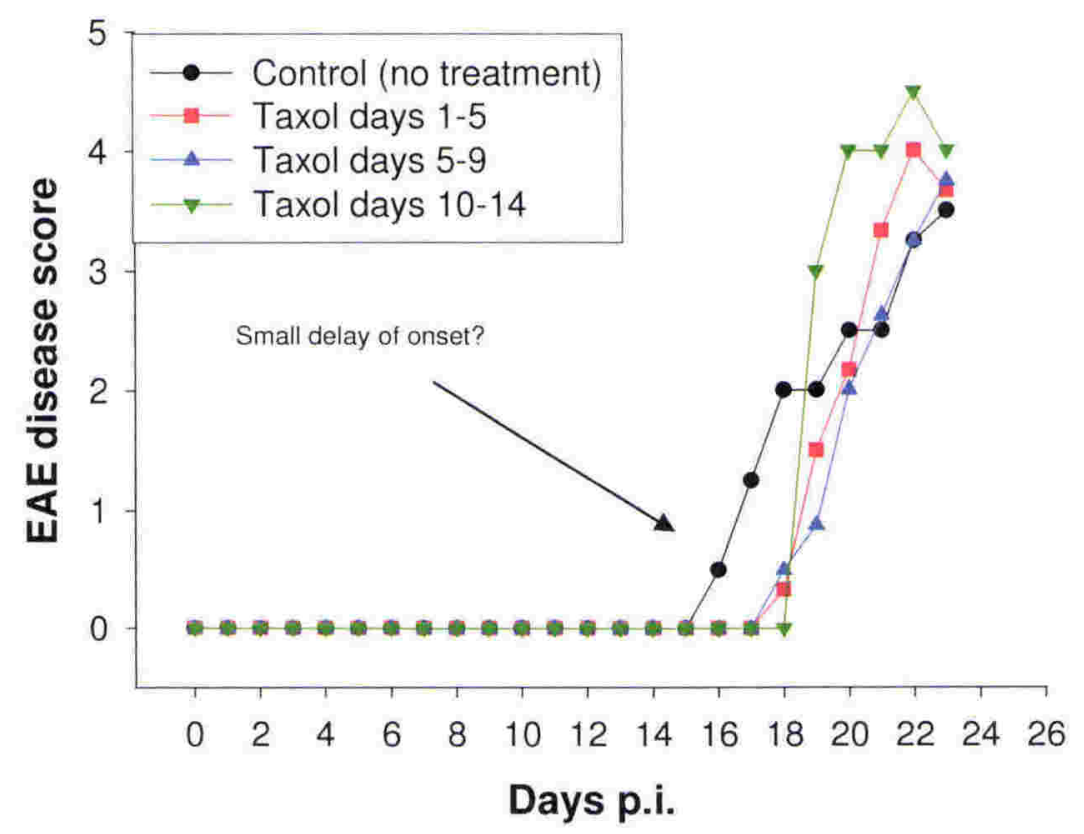

\section{Figure 5.1 Effect of Taxol on mice with EAE.}

Taxol (10 mg/kg) was administered to mice for 5 consecutive days starting 1 day (red squares; $n=3$ ), 5 days (blue triangles; $n=4$ ), or 10 days (green upside-down triangles; $n=1$ ) following EAE immunisations. Control animals were immunised, but did not receive Taxol (black circles; $n=2$ ). A two-day delay of onset occurred in the sick mice treated with Taxol. Shown are the results from sick mice only. The original study started with 5 mice/group. 


\subsubsection{Taxol at its MTD delays the onset of EAE when given in multiple daily doses.}

As shown above, low doses of Taxol $(10 \mathrm{mg} / \mathrm{kg})$ given daily produced a hint of a delay of disease onset for all three regimes. To maximise the disease-delaying potential of Taxol, the treatment doses were increased two-fold to the MTD. It was decided that the optimal period to treat mice with the MTD of Taxol was from days 0-4 p.i. in order to focus primarily on the initial proliferative events that kickstart the disease process. Treating mice with the MTD of Taxol $(20 \mathrm{mg} / \mathrm{kg})$ daily from days 04 p.i. resulted in a clear delay of disease onset (Fig. 5.2). Five immunised controls received Cremophor EL, the vehicle for paclitaxel, and showed no differences when compared to the untreated immunised controls (data not shown).

a

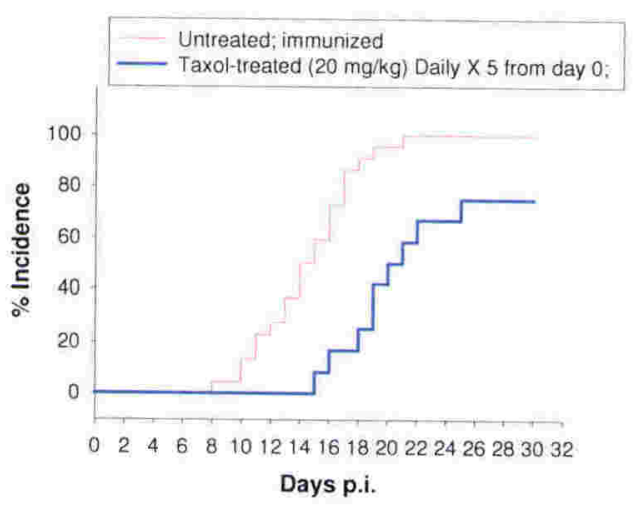

b.

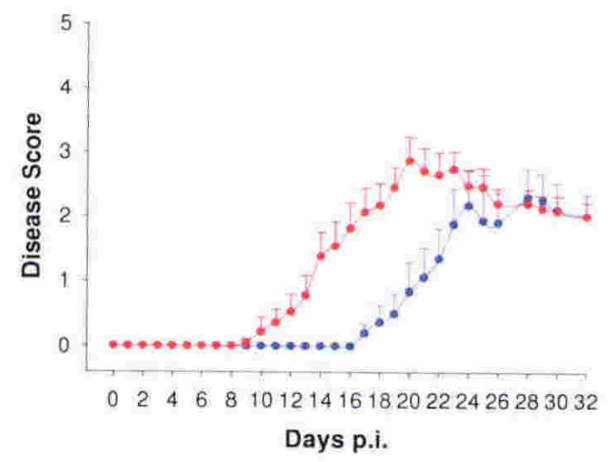

\section{Figure 5.2 Taxol at $20 \mathrm{mg} / \mathrm{kg}$ delays the onset of EAE.}

Mice immunised for EAE were left untreated (red; $n=22$ ) or treated with Taxol $(20 \mathrm{mg} / \mathrm{kg})$ for 5 consecutive days (blue; $n=12)$ immediately following immunisation. Shown are the average incidence (a) and disease score (b) in the days following immunisation. Error bars on (b) are SEM values. 
In immunized but non-Taxol treated animals, a slight weight loss is common in the days that follow immunisation (up to $5 \%$ of body weight). Taxol-treated immunised animals, however, exhibited a greater degree and duration of weight loss compared to immunised control mice (Fig. 5.3). Unexpectedly, 5 out of 17 MOG-immunised, Taxol-treated mice (29\%) died for no apparent reason between days 7 and 11 before any symptoms of EAE were evident. Non-immunised animals receiving Taxol (20 $\mathrm{mg} / \mathrm{kg}$ ) daily for 5 days were alive and healthy, with negligible weight-loss, for up to 14 days after the initial treatment $(n=7$; data not shown). These results suggest that, firstly, paclitaxel is effective at modifying EAE disease progression; secondly, that paclitaxel is well tolerated in C57BL/6 mice when administered to non-immunised animals, but an unknown toxicity occurs in mice that are both treated with paclitaxel and immunised for EAE.

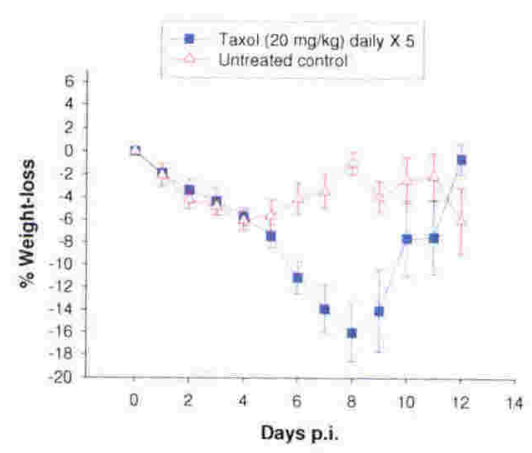

\section{Figure 5.3 Weight-loss from} Taxol-treatment.

Shown is the percentage of total weight-loss from $20 \mathrm{mg} / \mathrm{kg}$ Taxoltreated (blue squares; $n=12$ ) and untreated (red triangles; $\mathrm{n}=22$ ) immunised mice. The greater weightloss in the treated group was highly significant.

\subsubsection{Peloruside delays the onset of EAE.}

Since $20 \mathrm{mg} / \mathrm{kg}$ of Taxol successfully delayed the onset of EAE, it was hypothesised that peloruside might also have a similar effect due to its comparable microtubulestabilising properties. The higher solubility of peloruside in aqueous solutions allows 
Cremophor EL to be replaced by ethanol as the vehicle for delivery. An effective cancer-treating dose of peloruside, determined to be $10 \mathrm{mg} / \mathrm{kg}$ (Peter Northcote; personal communication), was dissolved in ethanol and administered in a $10 \%$ solution i.p. to mice immunised for EAE. Treatments were given daily on days 0-4 p.i. similar to the Taxol treatment regime given in Fig. 5.1. Peloruside delayed the onset of EAE and completely prevented disease in $20 \%$ of the animals treated (Fig. 5.4). Weight-loss from peloruside treatments was greater and more prolonged compared to the controls, but was slightly less than the weight loss seen with Taxol treatment at $20 \mathrm{mg} / \mathrm{kg}$. In contrast to the Taxol treatments, no deaths resulted from peloruside in MOG-immunized mice, suggesting that peloruside may be a better choice than Taxol for treating EAE. 
a

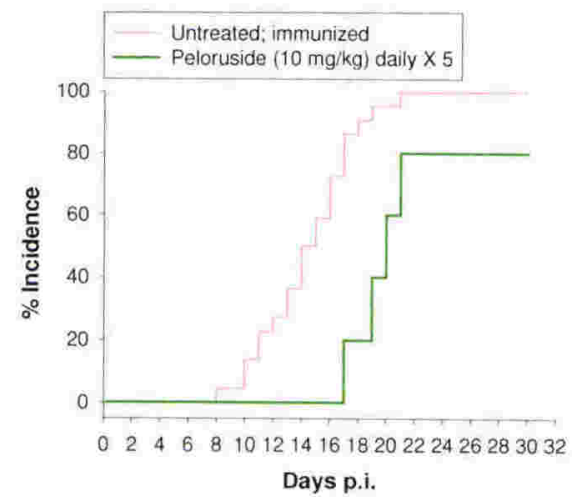

b

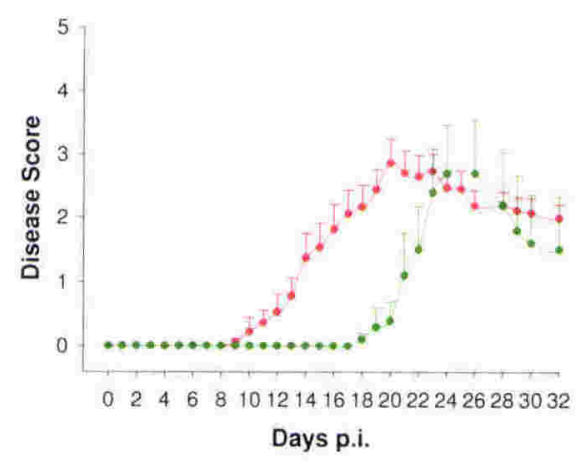

Figure 5.4 Peloruside at 10 $\mathrm{mg} / \mathrm{kg}$ delays the onset of EAE.

a-b) Peloruside (10 mg/kg; green) delayed the onset of EAE compared to controls (red). Weight-loss from peloruside treatments are presented in (c). Each graph is comprised of 2-4 experiments $(n=22$ for control group; $n=5$ for peloruside treated

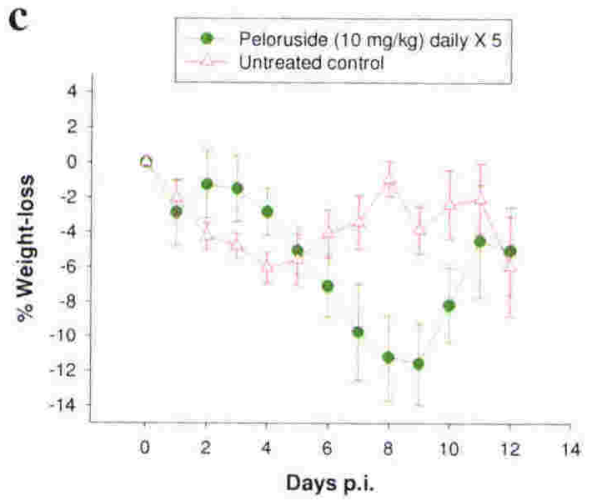
group). The weight loss following peloruside treatment was highly significant. 


\subsubsection{Taxol treatments after disease onset result in a high incidence of}

mortality.

Treating mice with $20 \mathrm{mg} / \mathrm{kg}$ Taxol daily for 5 days immediately after immunisation resulted in a delay of disease onset (Fig. 5.3), although there was also a $29 \%$ mortality observed in these experiments. To see if Taxol could be used to treat established disease, $20 \mathrm{mg} / \mathrm{kg}$ was administered to 8 mice daily for 5 days immediately following the first signs of disease, which normally occur at about 12 days p.i. Surprisingly, Taxol administered after disease onset had no significant effect on disease severity or duration (Table 5.2). Moreover, Taxol given after disease onset proved to be even more lethal to mice with EAE since 4 out of the 8 animals (50\%) died during, or shortly after, the first Taxol injection (Fig. 5.5). Because this dosing regime had little impact on disease and resulted in a high death rate, this pilot experiment was not repeated. 


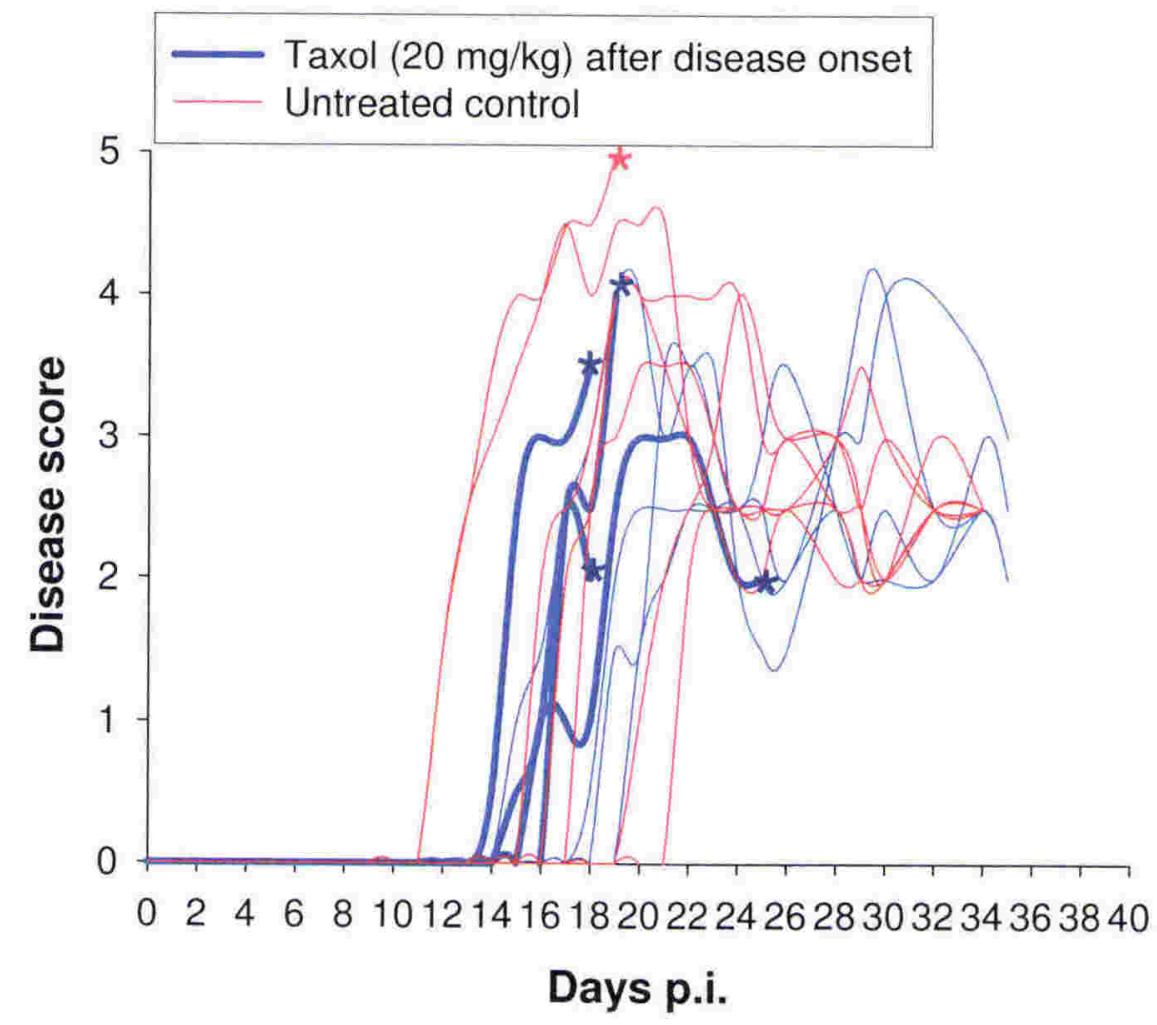

Figure 5.5 Effects of $20 \mathrm{mg} / \mathrm{kg}$ Taxol administered after disease onset.

Mice developing EAE were treated daily with $20 \mathrm{mg} / \mathrm{kg}$ Taxol for 5 consecutive days immediately after the first symptoms began to appear (blue lines; $n=8$ ). Untreated, immunised control mice from this experiment are shown for comparison (red lines; $n=7$ ). Each line represents the disease score over time for each individual mouse in this experiment. In the Taxoltreated group, 4 out of $8(50 \%)$ of mice died either during, or shortly after the Taxol treatments (thicker blue lines). Deaths in each group are represented by a star $\left(^{\star}\right)$. 


\subsubsection{Comparisons between Taxol and peloruside treatments}

Animals treated with Taxol (Fig. 5.2) and peloruside (Fig. 5.4) for 5 consecutive days following immunization with MOG/CFA showed similar trends in delaying the onset and severity of disease and in causing weight-loss (Fig. 5.6), thus suggesting that the modes of action were related to a common mechanism of the two drugs' action, either microtubule stabilisation or their anti-mitotic actions. The delay of onset from both drug treatments was highly significant compared to the untreated control group (Table 5.1).

There was a major difference, however, between Taxol and peloruside treated animals. An unknown toxicity was associated with Taxol treatments, but not peloruside treatments, administered before or during EAE, as mentioned above. Incidentally, the deaths that occurred from Taxol treatments initiated immediately following immunizations (experiments of Fig. 5.2) corresponded with the initial onset of disease in the untreated control group (Fig. 5.7). It is possible that there is a link between the known inflammatory effects of Taxol in vitro (Chapter 3 ) and the inflammation associated with the onset of EAE. 

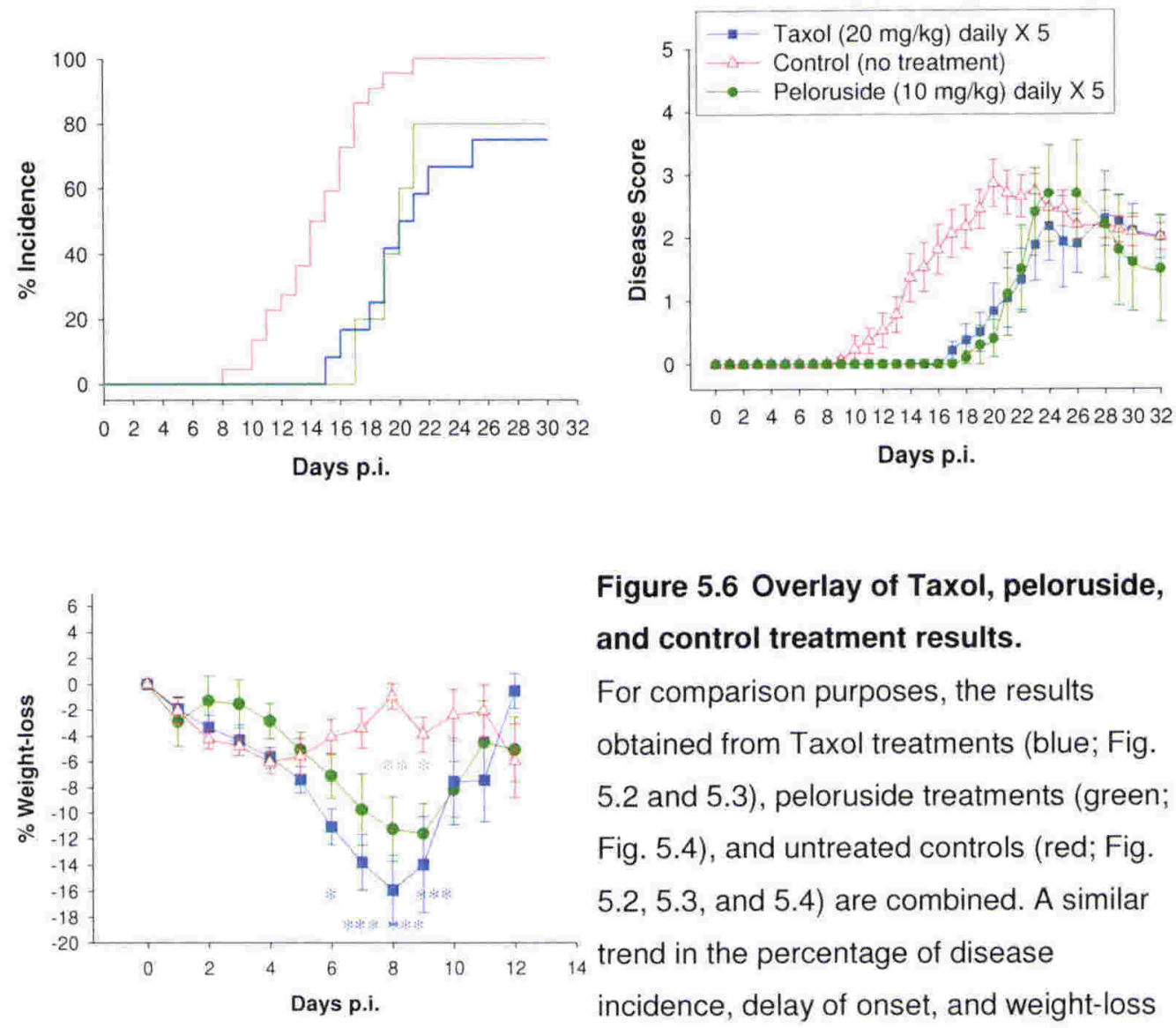

Figure 5.6 Overlay of Taxol, peloruside, and control treatment results.

For comparison purposes, the results obtained from Taxol treatments (blue; Fig. 5.2 and 5.3), peloruside treatments (green; Fig. 5.4), and untreated controls (red; Fig. 5.2, 5.3, and 5.4) are combined. A similar trend in the percentage of disease incidence, delay of onset, and weight-loss in drug-treated animals is evident. For weight loss: ${ }^{*} p<0.05,{ }^{* *} p<0.01$, and ${ }^{* \star *} p<0.001$ using two-way ANOVA with a Bonferroni post-test comparing drug treated animals to controls. There was no significance difference of weight loss between the two drug treated groups. 


\begin{tabular}{|llll|}
\hline$\underline{\text { Treatments }}$ & $\underline{\text { Incidence }}$ & $\begin{array}{l}\text { Average day } \\
\text { of onset (p.i.) }\end{array}$ & $\begin{array}{l}\text { Difference of means } \\
\text { from the control }\end{array}$ \\
$\begin{array}{l}\text { Untreated } \\
\text { controls }\end{array}$ & $22 / 22(100 \%)$ & $14.4 \pm 0.7$ & N/A \\
Taxol & $9 / 12(75 \%)$ & $19.4 \pm 1.0^{* \star *}$ & $5.0 \pm 1.3$ days \\
Peloruside & $4 / 5(80 \%)$ & $19.3 \pm 0.9^{* *}$ & $4.8 \pm 1.7$ days \\
\hline
\end{tabular}

\section{Table 5.1 Treatments of $20 \mathrm{mg} / \mathrm{kg}$ Taxol and $10 \mathrm{mg} / \mathrm{kg}$ peloruside} significantly delay the onset of EAE.

Single comparisons of the drug-treated and untreated control means were performed in each case using Student's $t$-test. The average day of disease onset was calculated by averaging the first day of clinical signs for each mouse in the group. Data are presented as the mean $\pm \operatorname{SEM}\left({ }^{\star \star} \mathrm{P}<0.01,{ }^{\star \star \star} \mathrm{P}\right.$ $<0.001$ compared to untreated control group). 


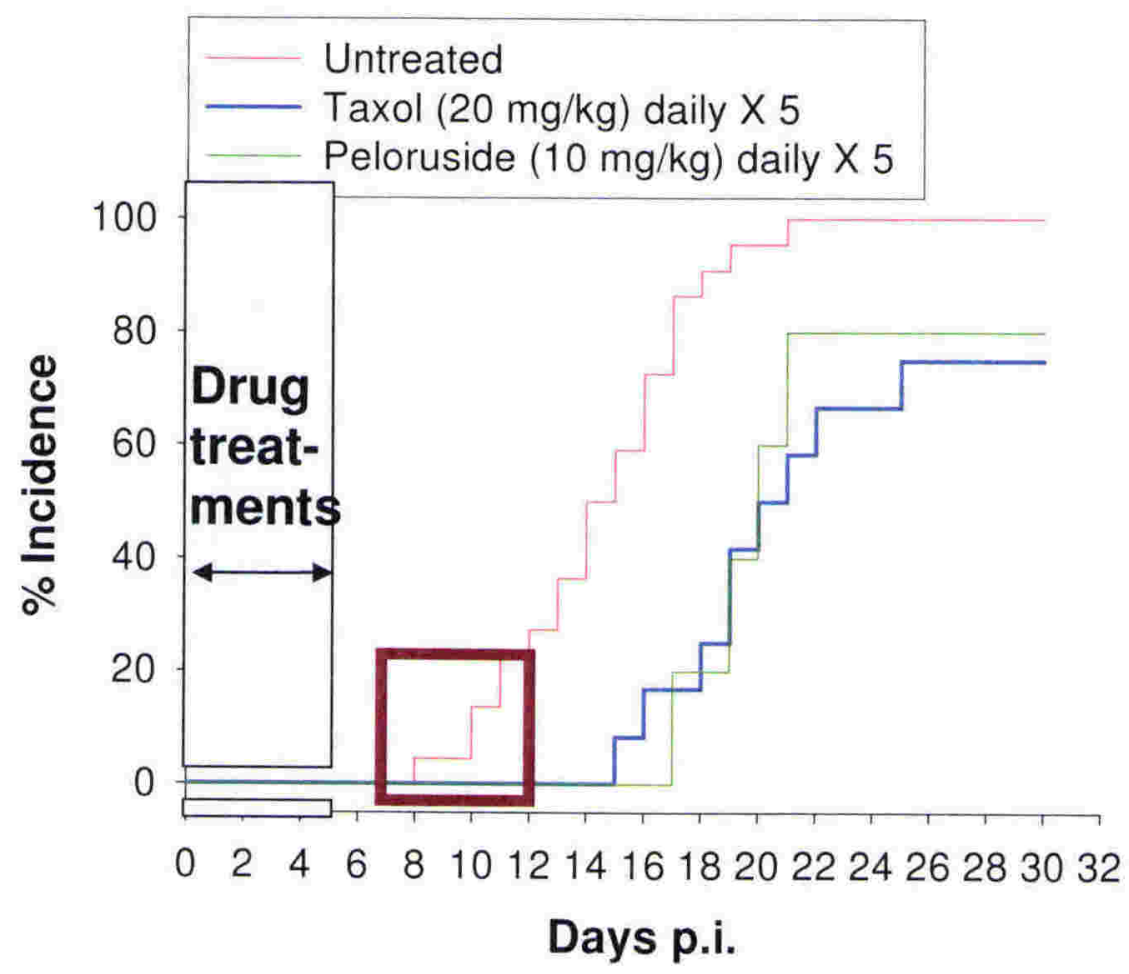

Figure 5.7 Toxicity of Taxol corresponds to initial onset of EAE symptoms. Taxol was toxic to 5 out of 17 mice when administered for 5 consecutive days immediately following immunisation for EAE. The time of toxicity corresponded to the time when the control mice (red) began to show symptoms of disease (dark red box) from days 7 to 12 . Animals treated with Taxol (blue) and peloruside (green) are shown for reference (data from Fig. 5.2 and 5.4). 
Several correlations were made when the data of the peloruside treatment group, the Taxol treatment group, and the control group were compared further (Table 5.2). The cumulative disease score was significantly lower in Taxol and peloruside treated groups compared to the controls. The cumulative disease score can give an indication of disease persistence; however, the significant differences resulting from the Taxol and peloruside treatments were primarily due to the delay of onset. There was no difference between the treated and control groups when comparing the peak disease scores, although Taxol-treated animals did spend significantly less time at peak disease compared to the control group, suggesting that Taxol administered prophylacticly may shorten the duration of disease. The average number of days spent at peak disease for peloruside-treated groups was not significant compared to the control group; however, the low sample number $(n=4)$ combined with the fact that one of the mice was at peak disease score for 8 days suggests that peloruside may actually shorten the duration of disease similar to Taxol, but this would have to be further clarified by additional experiments. Unfortunately, because of the limited stocks of peloruside available for these experiments, it was not possible to repeat these tests.

When Taxol was administered at the MTD for 5 consecutive days beginning at the start of disease onset, $50 \%$ of the mice died. In addition, there was no significant benefit with this therapeutic treatment regime when several disease parameters were compared to the control group, including the level of disease severity and the duration of disease symptoms (Table 5.2), suggesting that this treatment regime is substantially toxic, and is not beneficial to mice displaying symptoms of EAE. 


\begin{tabular}{|l|l|l|l|l|}
\hline Treatments & Controls & Taxol & Peloruside & $\begin{array}{l}\text { Taxol (upon } \\
\text { disease onset) }\end{array}$ \\
\hline $\begin{array}{l}\text { Cumulative disease score } \\
\text { at day 24 p.i. }\end{array}$ & $26.0 \pm 3.0$ & $8.3 \pm 3.0^{* \star \star}$ & $8.5 \pm 3.0^{\star \star}$ & $18.1 \pm 2.9$ \\
\hline Peak disease score & $3.8 \pm 0.2$ & $3.2 \pm 0.5$ & $2.8 \pm 0.8$ & $3.5 \pm 0.2$ \\
\hline Days at peak disease ${ }^{\dagger \dagger}$ & $4.9 \pm 0.4$ & $2.9 \pm 0.7^{\star * \star}$ & $5.0 \pm 1.1$ & $3.8 \pm 0.9$ \\
\hline
\end{tabular}

\section{Table 5.2 Comparisons between different drug treatments.}

Taxol (20 mg/ $/ \mathrm{kg})$ and peloruside (10 mg/kg) were given for 5 consecutive days immediately following immunisation, except where indicated. The cumulative disease score for each group was calculated by averaging the sums of daily scores for each mouse following immunisation. Values are presented as the \pm SEM $\left({ }^{* *} \mathrm{P} \leq 0.01,{ }^{* \star *} \mathrm{P} \leq 0.001\right.$; Student's $t$-test).

\subsubsection{Spinal cord lesions correlate to disease score in Taxol- and peloruside- treated mice.}

The course of the disease in mice with ensuing EAE was significantly altered by treating mice with Taxol or peloruside immediately after MOG-immunisation (Table 5.1). Although no difference in peak disease score was found (Table 5.2), differences in the number of lesions or sizes of lesions may have been present in the spinal cords of treated mice. To test for this possibility, the spinal cord was removed at day 30 p.i. from two representative mice from each group - one at the time of peak disease score (i.e., 4 or greater), and one that showed no symptoms or mild symptoms of disease. For comparison, spinal cords from a MOG-immunised, untreated mouse (disease score of 4.5 ; harvested day 20 p.i.) and a normal mouse (no EAE) were also removed. Tissues were stained with $H \& E$ to more easily identify lesions and cellular 
infiltrates (Fig. 5.8). Inflammatory foci were counted and given a score for each slide. As typically seen in EAE mice, the high degree of disease severity ensured that there would be a number of lesions of all sizes. Animals that did not get sick, or were mildly sick following the Taxol or peloruside treatment regime had no/few (respectively) noticeable lesions in their spinal cords (not shown). Animals with a severe score (i.e., 4 or greater) had spinal cords with multiple, but similar inflammatory foci of varying sizes (based on score) in the white matter parenchyma, regardless of whether the animal was untreated or treated with Taxol or peloruside. The total numbers of lesions in the representative slides from each group were comparable, with the Taxol-treated animal having a total of 67 lesions, pelorusidetreated animal having 60 lesions, and the untreated animal having 61 lesions. These results suggest that, once inflammatory cells have entered the CNS, disease progresses as normal regardless of the treatment. 

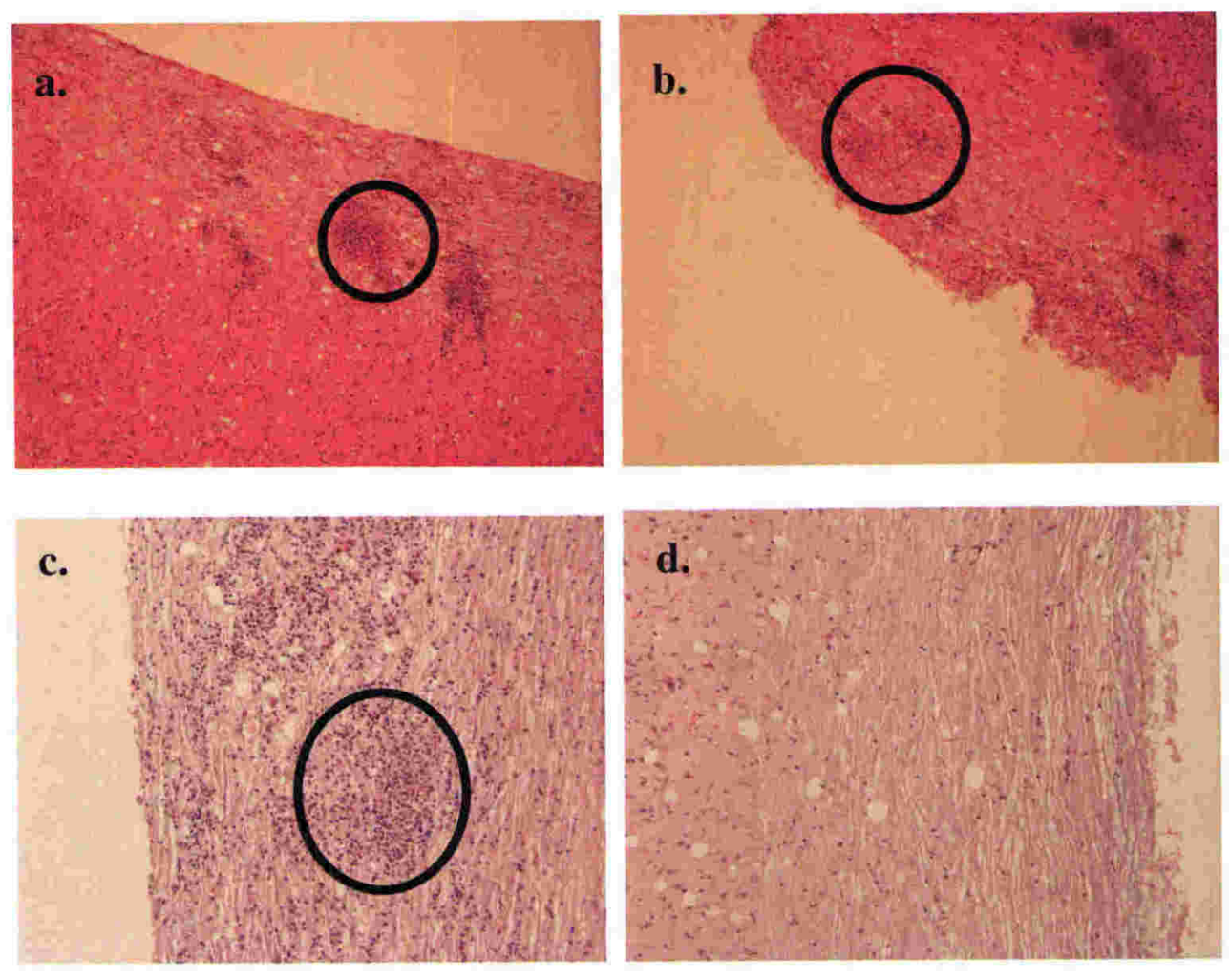

Figure 5.8 Spinal cords from drug-treated mice with active EAE.

Spinal cords were removed from MOG-immunised mice at peak disease score. Mice had been treated with Taxol at $20 \mathrm{mg} / \mathrm{kg}$ (a) or peloruside at 10 $\mathrm{mg} / \mathrm{kg}$ (b) for 5 consecutive days immediately following immunisation with MOG/CFA. An immunised, non-drug treated mouse spinal cord (c), and a normal spinal cord (d) are shown for comparison. All samples were stained with H\&E. Some typical severe lesions (i.e., score of 3 ) are circled for ease of identification.

\subsubsection{Re-stimulation of $\mathrm{LN}$ cells from Taxol and peloruside treated mice.}

To see if the delay of disease onset seen following Taxol and peloruside treatments was an effect of specific T cell reduction, DLN population distributions were determined by FACS analysis from drug-treated and vehicle-treated animals 30 days after MOG-immunisations (Fig. 5.9). Different types of T cells were considered for comparison. The $\mathrm{CD} 4^{+}$cells are considered to be the primary source of effector $\mathrm{T}$ 
cells (see review reference (El Behi et al., 2005)). The $\mathrm{CD} 8^{+}$cells have also been shown to have a role in EAE, but generally at a later time point after the initial onset of disease. The $\mathrm{CD} 25^{+}$population of cells contains $\mathrm{T}_{\text {reg }}$ cells, which are primarily associated with the recovery from EAE. Although the drug-treated percentages of all three LN cell populations were slightly lower than the vehicle-treated controls, the differences were not significant. This suggests that neither Taxol nor peloruside has a significant effect on LN cell proliferation, or that if LN cell populations were decreased, they have recovered by day 30 p.i. when the measurements were taken. 
CD4

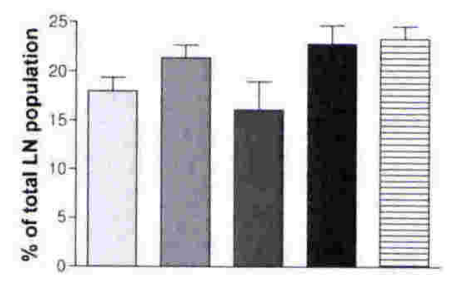

CD8

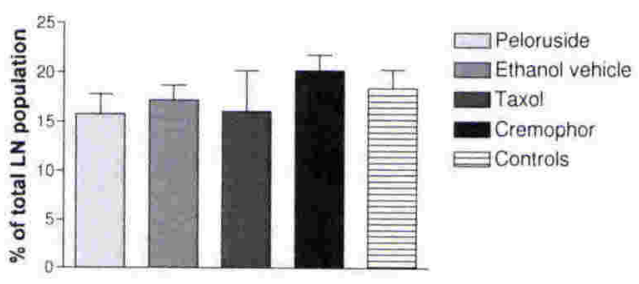

CD25

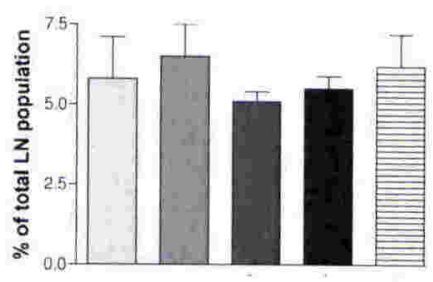

Figure 5.9 Populations of T cells from LN isolated from drug-treated mice with EAE.

Cells were isolated from the DLNs of EAE-immunised individual mice 30 days p.i. and stained for CD4, CD8, or CD25 expression. The \% of the specific $T$ cell subtypes in the total population of $L N$ cells was determined by FACS. No significant differences were observed between the drug-treated and vehicle-treated groups using the Student's $t$-test. The number of individual LN preparations for each treatment group were: peloruside $(n=5)$, ethanol $(n=8)$, Taxol $(n=3)$, Cremophor $(n=5)$, and the control $(n=7)$.

The proliferative capacity of MOG-reactive T cells, as well as the degree of activation, were also assessed to determine the extent, if any, of cell-specific cytotoxicity of Taxol and peloruside. DLN cells were cultured in media containing Con A to induce general proliferation, or MOG to induce MOG-specific proliferation, for $72 \mathrm{hr}$. IFN- $\varepsilon$ levels were measured in the supernatants from each 
group by ELISA, and proliferation was detected using the CyQUANT assay. The results from the CyQUANT assay show that there was no proliferation in any group in response to MOG peptide (Fig. 5.10 a). Although a memory response would typically be present 30 days after an initial immunisation, the results presented here are common in re-stimulation assays ( 30 days p.i.) with cells from EAE mice according to Dr. Thomas Backstrom and Dr. Andrea McNeill (Malaghan Institute of Medical Research, Wellington, NZ), and Dr. Anne La Flamme (Victoria University of Wellington, NZ) (personal communications), possibly due to anergy. Con A, however, did induce proliferation in each group, albeit low levels in LN cells from untreated and peloruside treated animals, indicating that the LN cells were capable of proliferation (Fig. 5.10 b). These results may not be reliable, however, since the CyQUANT assay requires a low cell seeding number to maintain a linear detection range. For Con A-stimulated or MOG-stimulated T cell proliferation to occur, the cells must be within close proximity to APCs in the culture, which is less likely to occur with a low seeding density. This situation was unavoidable, since the total number of LN cells isolated from each mouse was limited, thus preventing the use of the much preferred MTT proliferation assay.

When LN cells from each group were cultured with MOG peptide, IFN- $\varepsilon$ was produced, but levels of this cytokine were not significantly different between groups (Fig. 5.10 c). The Con A control shows that the LN cells do produce IFN- $\varepsilon$ when stimulated, and that the overall number of IFN- $\varepsilon$-producing cells is not different between groups (Fig. 5.10 d). Although no differences were observed between the drug-treated groups and the untreated control group, it is possible that there would 
have been a difference prior to day 30 in proliferative responses and antigen-specific re-stimulation responses, but these may have been obscured at this later time point.
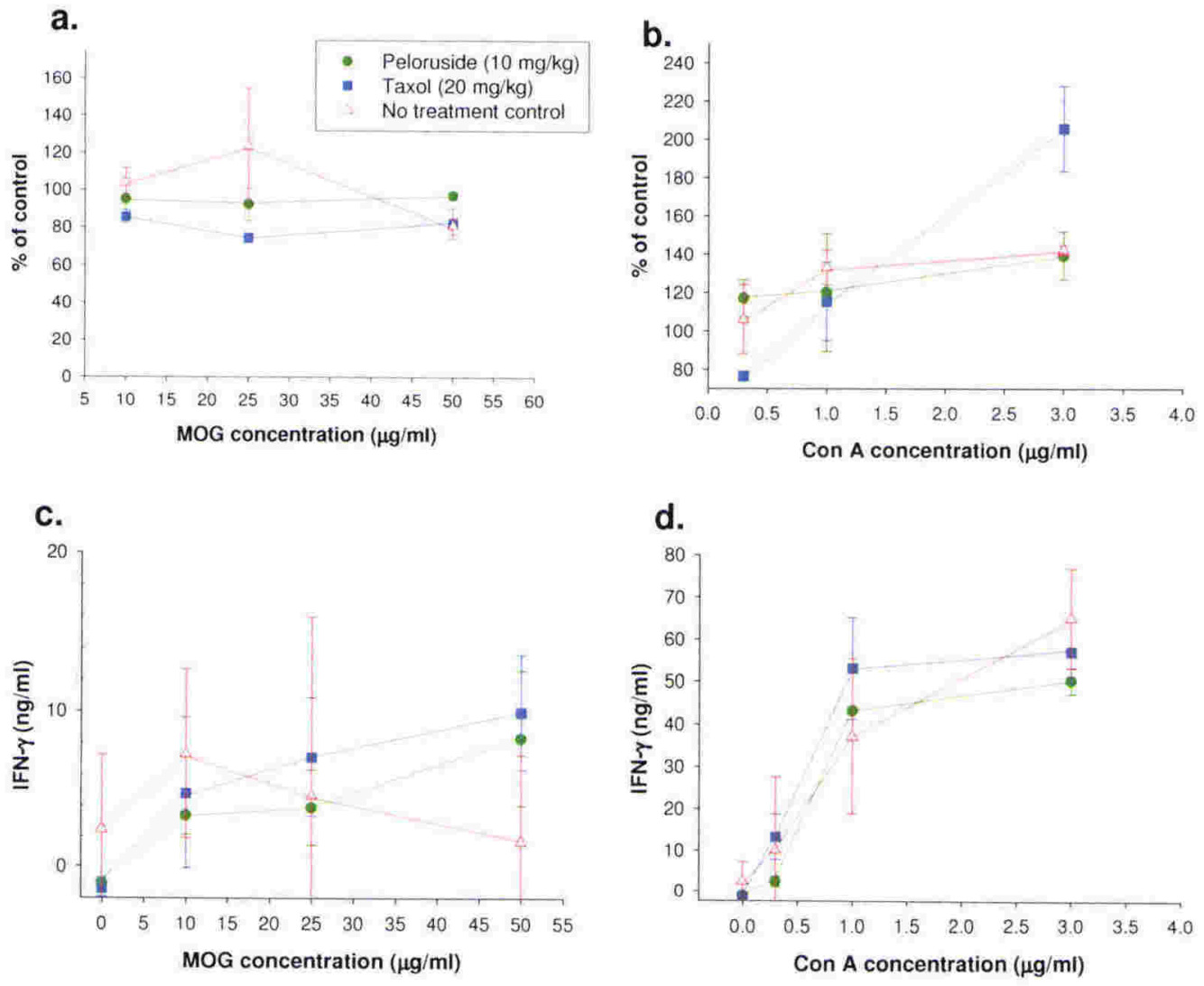

Figure 5.10 Re-stimulation of LN cells that were isolated 30 days p.i. EAE-immunised mice were treated with Taxol (blue squares; $n=3$ ), peloruside (green circles; $n=4$ ), or left untreated (red triangles; $n=2$ ) for 5 consecutive days immediately after immunisation. DLN cells from each mouse were isolated and cultured individually for $72 \mathrm{hr}$ in the presence of MOG $(a, c)$ or Con A (b, d). Proliferation was determined by the CyQUANT assay ( $\mathrm{a}-\mathrm{b})$, and IFN- $\leq$ was determined by ELISA (c-d). Panel (a) shows that no proliferation occurred in response to specific-antigen stimulation, but the cells were capable of proliferation, as shown in panel (b). Similarly, panel (c) shows that IFN-s is produced in response to MOG; however, the differences were not significant (based on Student's $t$-test). 


\subsubsection{Taxol does not affect $\mathrm{CD}^{+} \mathrm{T}$ cell proliferation.}

The administration of Taxol during the 5 days following MOG-immunisation delays the onset of EAE, but does not significantly alter the lymphocyte populations or their cytokine responses by the time disease recovery is underway. To specifically determine if the effects of Taxol are anti-proliferative as hypothesised at the start of this study, the effect of Taxol on in vivo proliferation was investigated. To assess proliferation in vivo, CFSE-labeled cells were transferred from 2D2 mice, which are transgenic for a majority proportion of T cells expressing a MOG-specific TCR, into WT C57BL/6 mice. Because CFSE is a fluorescent cytoplasmic dye that is equally divided amongst daughter cells during mitosis, the in vivo proliferation of these MOG-specific cells could be determined by flow cytometry as a dilution of the fluorescent label with time. One day following the transfer, mice were immunised with MOG/CFA and immediately treated with Taxol $(20 \mathrm{mg} / \mathrm{kg})$ for 5 consecutive days. The DLN cells were isolated one day after the final Taxol treatment and analysed for proliferation by FACS. Surprisingly, Taxol did not have any effect on the in vivo proliferation of the CFSE-labeled T cells (Fig. 5.11), suggesting that the delay of EAE onset in response to Taxol treatments is not due to the inhibition of proliferation of MOG-specific T cells. 


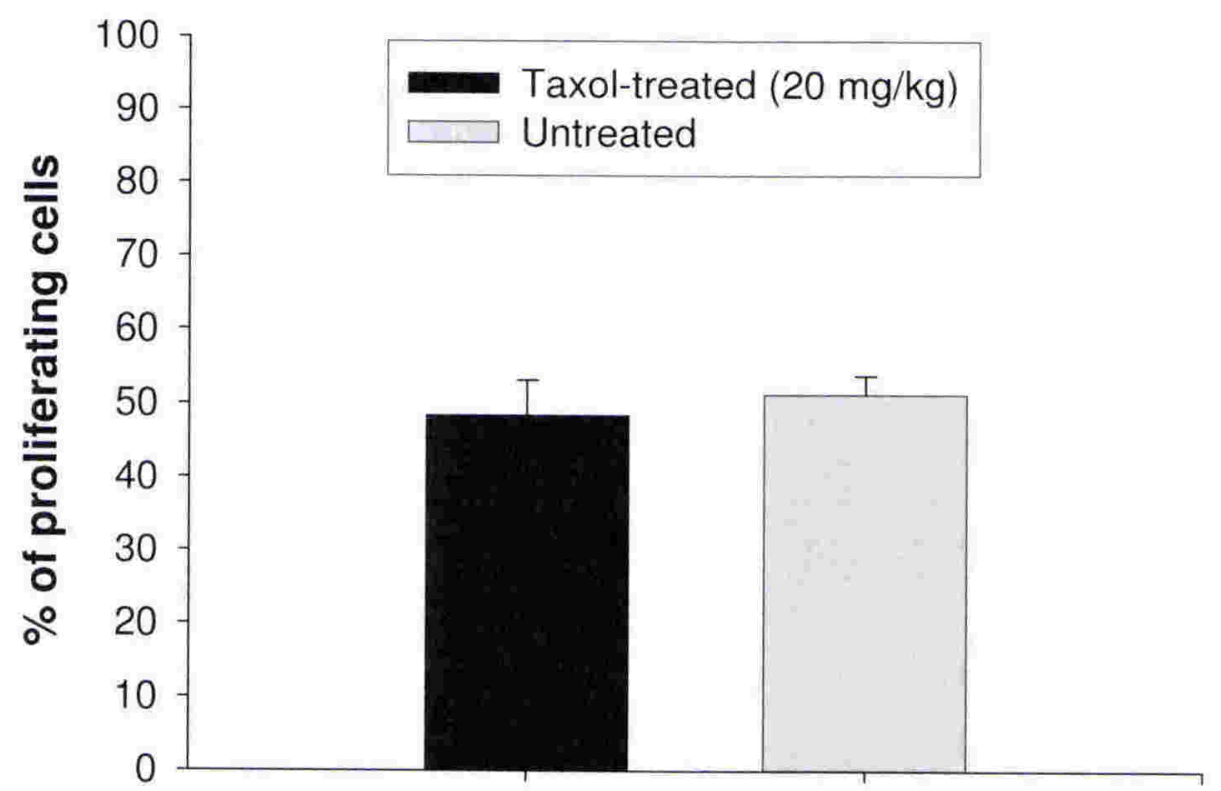

Figure 5.11 Taxol does not affect MOG-specific $\mathrm{T}$ cell proliferation.

LN cells from 2D2 mice were stained with CFSE and transferred to wildtype mice, which were then immunised for $\operatorname{EAE}(n=20)$ and treated with $20 \mathrm{mg} / \mathrm{kg}$ Taxol $(n=10)$, or were left untreated $(n=10)$. Control mice $(n=$ 6) received CFSE-labeled cells, but were not immunised for EAE and were left untreated (data not shown). For the treated group, Taxol $(20 \mathrm{mg} / \mathrm{kg}$ ) was administered daily for 5 days immediately following immunisation. DLN cells were isolated from each mouse within these groups and analysed by FACS individually to detect in vivo proliferation of the cells. The percent of total CFSE + cells that had undergone at least one cell division are shown ( $n=10$ from two separate experiments). A further explanation of how this graph was created can be found in Appendix C. 


\subsection{Discussion}

During the course of EAE, a specific sequence of cellular events must occur for clinical disease to develop. For example: following the initial immunisation, DCs take up antigen and become activated, then they migrate into the DLN where they induce MOG-reactive T cells to undergo clonal expansion. This all occurs during the first 5 days p.i. Days 5-9 p.i. encompass what is considered to be peak proliferation in the periphery (Targoni et al., 2001). Throughout days 10-14 p.i., activated T cells begin to migrate across the BBB where ensuing damage to the brain and spinal cord occur (Juedes et al., 2000; Targoni et al., 2001), subsequently causing animals to show symptoms of disease. Since Taxol, when administered on days 6, 8, and 12 had no effect on EAE development (Chapter 4), it was thought that by targeting these specific 5-day periods, Taxol would then have an effect on the EAE disease course. Although the disease incidence was low in the particular set of experiments used to test these treatment regimes, treatments of Taxol $(10 \mathrm{mg} / \mathrm{kg})$ with all three proposed dosing regimes caused what appeared to be a small delay in disease onset of about 2 days, suggesting that Taxol can affect EAE disease progression if the dosing regime is optimised.

When the treatment regime was altered to 5 consecutive daily treatments with Taxol at its MTD of $20 \mathrm{mg} / \mathrm{kg}$ given immediately after immunisation, there was a clear and significant delay of disease onset, but also a $29 \%$ mortality rate. Neither mortality, nor a significant delay of onset, was seen in animals treated with $10 \mathrm{mg} / \mathrm{kg}$ with the same regime, but an unexpected low disease incidence may have obscured the outcome of this experiment. Most interestingly, peloruside delayed the onset of EAE in a manner similar to high-dose Taxol, but without causing any deaths. The 
mechanism that causes the delay of onset appeared to be related to microtubulestabilisation, or at least the anti-mitotic mechanism of the two drugs, since both drugs had nearly identical disease modifying effects when administered at the active doses.

The subsequent weight loss, disease scores, and delay of onset of EAE in mice treated with both Taxol and peloruside were nearly indistinguishable (Fig. 5.6). Other disease parameters were also similar between the two treated groups, including the cumulative disease scores and the peak disease scores (Table 5.2). A major difference between the Taxol-treated and peloruside-treated groups is that $29 \%$ of the Taxol-treated animals died between days 7-12 p.i.; whereas, peloruside was not lethal to any of the animals. Control animals that did not receive the initial immunisation, but did receive Taxol treatments, were largely unaffected based on appearance and weight, and none of these control animals died. This is a strong indication that Taxol itself is not toxic with this dosing regime, but for reasons unknown at this time, the combination of Taxol in MOG-immunised mice proved to be fatal in about one-third of the animals. Thus, there is some adverse interaction between the Taxol effect and the MOG-immunisation effect in some of the animals. Interestingly, the Taxol-induced deaths occurred between days 7 and 12 p.i., which coincides with the normal onset of EAE in untreated, but immunised control mice (Fig. 5.7). Additionally, mice treated with Taxol at the time of disease onset also showed high lethality with 4 out of 8 animals dying either during or shortly after the 5-day treatment regime. Combined with the lethality observed by Cao et al (2000) after Taxol treatments in rats with EAE, these data suggest that there is an inflammatory cross-reaction when Taxol is given to animals in the induction and effector phases of EAE, especially since this toxicity is not observed in animals 
treated with peloruside, which lacks the inflammatory stimulation effects. One cannot rule out the possibility that Taxol-treatments caused the deaths in mice with the most aggressive disease. This scenario could potentially cause the data to be skewed making it appear that a delay of onset had occurred. A much more extensive investigation would be needed, however, to positively link Taxol administration with enhanced mortality in mice and rats.

The common action of peloruside and paclitaxel is the ability to prevent microtubule depolymerisation. The delay of EAE in response to altered microtubule function is further supported by experiments with colchicine, a microtubule-depolymerising drug, which similarly altered the development of EAE (Lyons et al., 1986). As shown previously in Chapter 3, however, paclitaxel differs from peloruside by causing inflammatory events to occur due to TLR4-complex stimulation. Because a similar delay of onset was observed in mice with EAE that were treated with either Taxol or peloruside, it can be tentatively concluded that the delay of onset is due to microtubule stabilisation and/or its downstream effects (mitotic arrest and apoptosis), and not because of the LPS-mimicking characteristics of Taxol.

While these results clearly indicate that microtubule-stabilising drugs delay the onset of EAE, the mechanism responsible for this delay is not known. The most notable and practical function of microtubule-stabilising drugs is their use in cancer treatment. Simply put, these drugs kill rapidly dividing cancer cells. Therefore, T cells that become activated during the initial 5 days after MOG-immunisation may succumb to the anti-mitotic effects of both drugs, which would ultimately delay the onset of disease until the Taxol is cleared. Once Taxol is no longer affecting T cell 
proliferation ( $24 \mathrm{hr}$ after the last treatment), the MOG-reactive T cells may begin to successfully proliferate, eventually leading to the development of EAE, albeit later than normal. This theory was supported by the fact that re-stimulation assays showed that there were no differences between the treated and untreated groups regarding both MOG-specific proliferation and IFN- $\varepsilon$ production (Fig. 5.11). This finding, however, may be due to the timing of the assays (day 30 p.i.), as it is not unusual to see a limited MOG-re-stimulated T cell response after 30 days have passed since the initial immunisation. Assessment of responses at a time when Taxol directly exerts its effect is required and would form the basis of future studies in this laboratory.

To more accurately determine whether Taxol was inhibiting proliferation of MOGreactive $\mathrm{T}$ cells during the induction phase of disease, transgenic $\mathrm{T}$ cells specific for MOG peptide were stained with CFSE and transferred to mice a day prior to MOG immunisation and the subsequent Taxol treatments for 5 consecutive days. It was clear that during the time of the Taxol treatments, proliferation was occurring in these CFSE-labeled cells, thus proving that Taxol did not inhibit in vivo proliferation of these autoreactive T cells during the induction phase of disease. This was very surprising because low $\mathrm{nM}$ concentrations of paclitaxel are cytotoxic to proliferating splenocytes in vitro (Chapter 3 ). It is possible that the reason cytotoxicity did not occur in vivo was due to the P-gp efflux pump, which is responsible for multi-drug resistance in multiple cancer cell lines. Although the P-gp pump is active in vitro (Gaitanos et al., 2004), the cells are persistently exposed to the drug when it is added to a culture. Taxol concentrations in vivo, on the other hand, are initially high, but rapidly decrease over the course of hours (Kim et al., 2001). The P-gp pumps only need to keep clearing Taxol from the cells for hours before the blood-Taxol 
concentrations are reduced to survivable levels. This may explain why Taxol did not kill the CFSE-labeled T cells in vivo; however, this absence of an effect on T cell proliferation leaves the question of how Taxol and peloruside delay EAE unanswered.

It was shown previously in Chapter 3 that both paclitaxel and peloruside decrease TNF- $\varepsilon$ production in MØ exposed to inflammation-inducing LPS. Blocking the effects of TNF- $\varepsilon$ has been shown to prevent an entire cascade of inflammatory cytokines (Feldmann, 2002; Feldmann et al., 1996), giving support to the possible immunomodifying effects of Taxol in EAE. It has also been shown that functional microtubules are necessary for antigen processing and presentation by DCs and MØs (Peachman et al., 2004). In this particular study, the authors specifically identified paclitaxel as a dramatic reducer of antigen processing/presentation in DCs due to microtubule stabilisation. Because of the diverse roles of microtubules in cellular functions, there are many other plausible explanations for the disease-delaying effects of Taxol; however, it is clear, based on the studies with CFSE-labeled MOGspecific T cells, that the most likely scenario - preventing proliferation of $\mathrm{T}$ cells does not occur with Taxol treatments. 


\section{Chapter 6: General Discussion}

\subsection{Summary}

In the present study, it has been shown that the microtubule-stabilising drugs, paclitaxel and peloruside, are capable of modifying the progression of EAE. Hence, paclitaxel and peloruside have the potential to impact MS, but further research is needed to optimise the disease modifying effects.

The primary hypothesis of this project was that microtubule-stabilising anti-mitotic compounds would inhibit proliferation in the autoreactive $\mathrm{CD} 4^{+} \mathrm{T}$ cells that mediate EAE and MS. Paclitaxel, being inexpensive and readily attainable, was chosen as the ideal microtubule-stabilising drug candidate. A novel microtubule-stabilising compound, peloruside, was also tested as an alternative to paclitaxel to identify immunomodifying activities, and to act as a microtubule-stabilising control. It was a natural approach to initiate the present studies by showing that $\mathrm{T}$ cells are susceptible to the anti-mitotic activities of both paclitaxel and peloruside in vitro. Because peloruside is one of several cytotoxic compounds to be isolated from the same sponge species, including mycalamide and pateamine, these three compounds were assessed for their direct effects on $\mathrm{T}$ cell proliferation and activation. Pateamine has been found to be a protein synthesis inhibitor that blocks the function of the initiation factor, eIF4A (Bordeleau et al., 2005). Mycalamide, like pateamine, is a protein synthesis inhibitor but interacts directly with the ribosome (Burres and Clement, 1989). Although out of the scope of the present study, mycalamide exhibited immunosuppressive activities that warrant further investigations. More relevant to the present study, peloruside and paclitaxel were both cytotoxic to Con A-stimulated 
splenocytes, giving the first indication that proliferating $\mathrm{T}$ cells succumb to these anti-mitotic drugs.

The initial experiments identified the effects of paclitaxel and peloruside on T cells, but EAE lesions, although mediated by $\mathrm{CD} 4^{+} \mathrm{T}$ cells, are largely caused by the phagocytic activities of MØ. Interestingly, paclitaxel is known to stimulate the TLR4 complex in murine MØ similarly to LPS, inducing the production of inflammatory mediators. These LPS-mimicking activities of paclitaxel could have presented a major problem in animal studies, especially since the initiating events of EAE are largely enhanced by the TLR-stimulating effects from the CFA adjuvant. Peloruside, on the other hand, is known to bind to a different region of tubulin from paclitaxel (Gaitanos et al., 2004; Jimenez-Barbero et al., 2006; Pineda et al., 2004). Since both peloruside and paclitaxel have a similar mode of action, these drugs were assessed for inflammatory-inducing activities in BMMØ. Unlike paclitaxel, peloruside did not induce BMMØ production of inflammatory cytokines. This finding was not surprising because of the distinct microtubule-binding characteristics. Other studies have found that the TLR4-stimulating effects of paclitaxel are unique to this drug (Kirikae et al., 1996; Ojima et al., 2003), and that other microtubule-stabilising compounds without LPS-mimicking effects could also potentially serve as alternative microtubule-stabilising compounds to paclitaxel (Muhlradt and Sasse, 1997). Many of these other compounds, however, are expensive and not easy to source, especially in the quantities needed for in vivo studies.

The inflammation-causing properties of paclitaxel were an ongoing concern. Because EAE immunisations produce an inflammatory environment, it was important to 
identify any synergistic inflammatory effects from a combination of paclitaxel and LPS. Both paclitaxel and peloruside were cultured with BMMØ in the presence of LPS, and surprisingly, both compounds decreased the production of a key inflammatory mediator TNF- $\varepsilon$, as well as NO. It is not known why paclitaxel displays these paradoxical effects of causing the release of inflammatory cytokines, yet preventing their production in BMMØ in the presence of LPS. Although the mechanism behind these anti-inflammatory responses is yet to be resolved, these results showed that peloruside and paclitaxel may actually reduce inflammation, as well as exhibiting anti-mitotic functions. The significance of this finding is highly valuable in the context of treating an inflammatory autoimmune disease, such as EAE.

A previous study by Cao et al. (2000) found that Taxol delayed the onset of EAE in actively immunised Lewis rats. The authors observed that the most effective Taxol treatment regime for delaying onset unfortunately also caused the deaths of 11 out of 15 rats. After further review of this study, the observations made by these authors lead me to speculate that the TLR4-complex-stimulating function of Taxol may have contributed to the lethality in these rats by enhancing the production of inflammatory cytokines in an environment where severe inflammation already exists. Attempts were made to repeat these experiments in C57BL/6 mice, and to identify any inflammatory effects of Taxol in vivo. The treatment regime borrowed from Cao et al. (2000) involved i.p. injections of Taxol at concentrations as high as the MTD on days 6,8 , and 12 following MOG-immunisation. Although the mice tolerate these doses well, these treatments did not modify EAE disease progression. There may be many reasons why there was no disease-altering effect, including such possibilities 
as high P-gp efflux pump expression on T cells, differences in EAE progression and Taxol clearance rates in rats versus mice, or autoimmune cell proliferative "windows" occurring in the 2 and 4 day periods between Taxol treatments in mice. The activity of the P-gp efflux pump was not specifically tested, but methods could be employed to identify this activity such as the use of rhodamine 123 in colourimetric assays. Despite attempts to rule out the use of Taxol as a potential therapeutic for EAE, these studies did not provide sufficient evidence to suggest that this drug has adverse effects in mice due to its LPS-mimicking activity. At this point in the project it was decided that Taxol would continue to be the drug of choice to optimise in order to modify EAE, since the evidence from Chapter 4 suggests that TLR4-stimulating activity would not be a major factor.

Based on the evidence from Chapter 4, it was determined that disease-modifying effects of paclitaxel as seen in Lewis rats (Cao et al., 2000) did not occur with the same treatment regime when administered to mice. As mentioned earlier, there are several possibilities that may explain why no effect on disease was seen. To overcome these possibilities, a new treatment regime was proposed, which involved daily administrations of Taxol during the time periods encompassing key cellular events in the initiation of EAE. Initial attempts to modify the onset of EAE with a new Taxol regime were hampered by low EAE incidence in the groups. Other factors also could have contributed to the limited response, including the quantity of Taxol administered to each mouse. To exclude this possibility from affecting further experiments, Taxol doses were lifted to the MTD. In addition, administrations of Taxol were focused on the initial days immediately after MOG-immunisation, with the thought that it could directly target the initial proliferation of MOG-reactive T 
cells. When these experiments were performed, a significant delay of onset occurred, although there was concomitant cytotoxicity. This experiment confirmed what Cao et al. (2000) found in rats with paclitaxel, and now raises a few questions: can all microtubule stabilising compounds affect EAE development? If so, what is the mechanism?

Peloruside, our alternative microtubule-stabilising drug to paclitaxel, also successfully delayed the onset of EAE in mice when administered with the same regime as the disease-delaying doses of paclitaxel. Moreover, the Taxol-related toxicity did not occur with peloruside treatments. This peloruside-induced delay of onset is highly important, as it suggests that the disease-modifying effects of both Taxol and peloruside are related to their primary mode of action - the disruption of normal microtubular function. The primary hypothesis of this project was that microtubule-stabilisation causes proliferating cells to apoptose, thus inducing cell death in the effector $\mathrm{CD}^{+} \mathrm{T}$ cells that mediate EAE. This, however, was not the case, since autoreactive $\mathrm{CD}^{+} \mathrm{T}$ cells continued to proliferate in vivo after the administration of disease-modifying Taxol treatments. Limitations of peloruside supply prevented further experimentation in vivo, so it remains to be seen if peloruside also has no effect on $\mathrm{T}$ cell proliferation in vivo. Although the exact mechanism of disease modification remains unanswered, the known microtubulestabilising functions of both compounds are likely to be the root cause.

It is important to mention that a micellar formulation of paclitaxel was used in phase II clinical trials to treat MS (Angiotech_Pharmaceuticals, 2002). The results of these trials showed no significant effects between 174 paclitaxel-treated and placebo- 
treated subjects. In these studies, patients with secondary-progressive MS were treated with paclitaxel once every 4 weeks for 6 months. This is a confusing approach, since the cytotoxic effects of paclitaxel are a result of exposure time to the drug over a threshold concentration (Helson et al., 1993). When these trials began, the research by Cao et al. (2000) was the only published animal study identifying possible immunomodifying effects in a MS-like disease. As I have shown here, Taxol should not be dismissed as a failed MS therapy. This example simply highlights the fact that further EAE animal studies are critical to determine the mechanism behind the modification of disease in order to devise a better treatment strategy for MS patients.

\subsection{Future Directions}

While peloruside served as a control throughout this project, it is important to consider that peloruside is in limited supply and was only administered to 5 mice in total. The amount of peloruside used in these experiments was $1 / 10^{\text {th }}$ of the world's known supply of peloruside at the time these experiments were performed. When peloruside supplies are not as precious, further studies can be undertaken to develop this drug as a potential treatment for MS.

There are many roles for microtubules during normal cell function, and different cells utilise microtubules for various reasons. In DC, for example, the microtubulestabilising drug docetaxel has been shown to alter chemotactic motility (Nakashima et al., 2005), which would have major implications during the initial T cell priming events of EAE. Another study has shown that normal microtubule functions are required for antigen presentation and processing in $\mathrm{DC}$ and $\mathrm{M} \varnothing$, and paclitaxel was 
shown to alter antigen presentation in these cells (Peachman et al., 2004). The current project focused on T cells, but it is clear that other immune cells may have had impaired functions from Taxol and peloruside treatments. Introducing confocal microscopic detection methods, including total internal reflection fluorescence (TIRF), could help determine other immune cell responses to Taxol and peloruside, such as an impaired secretory pathway or altered migratory capacity.

The dosing regime of Taxol and peloruside that delayed the onset of EAE, although successful, was not fully optimised. As mentioned in Chapter 5, there were several different time periods that could be targeted in order to maximise the diseasemodifying effects. In addition, the dose of Taxol used in the disease modifying experiments was pushed to its limits in order to reduce the possibility that the quantities of drug given to mice were too low to see an effect. In fact, less Taxol may still modify disease, and it might also reduce toxicity. In addition, there is no reason why daily drug administrations cannot be extended for periods longer than 5 consecutive days, so long as weight-loss and toxicity do not become an issue. Taken together, the treatment regime can be modified for optimal disease-modifying results without the adverse side-effects.

The EAE model used throughout this project was the C57BL/6 model of disease. This particular model of EAE is useful to establish an early intervention during the initial phases of disease; however, most MS patients do not know they will get the disease until they have had it for some time. At that point, it is too late for early intervention. Different EAE animal models, such as the relapsing-remitting SJL mouse model, may be more appropriate for testing a potential therapy for the most 
common MS phenotype, relapsing-remitting disease. There are many more animal models of EAE that have characteristics more closely related to certain MS subtypes. Exploring the effects of microtubule-stabilising drugs in other EAE animal models may be a more effective way to establish a crossover link to a MS therapy.

An interesting, and unexpected effect from the Taxol treatments was the toxicity that accompanied the onset of EAE. This detrimental interaction may have a broader importance regarding the treatment of patients with Taxol when presenting with general inflammation. Although the TLR4-stimulating activities of paclitaxel are limited to murine animals, paclitaxel and other taxane derivatives have been linked to the production of another inflammatory mediator, COX-2, in human monocytes (Cassidy et al., 2002). Repeating the Taxol treatment regime in MOG-immunised TLR4 -/- mice may elucidate the mechanism by which the toxicity occurs, thus ruling out contraindications for Taxol treatments in humans.

Taxol is a widely used drug with the potential to treat MS, and peloruside, also having potential as a MS treatment, is being isolated in increasing quantities each year. Taxol, as shown here, has its drawbacks. But it is only a matter of time before peloruside will become widely available, which would allow further studies to take place in the EAE animal model of disease. Hopefully, the project presented here gives insight into using anti-mitotic drugs as treatments for $\mathrm{CD}^{+} \mathrm{T}$ cell-mediated autoimmune disorders, which may lead to another treatment option for MS patients around the world. 


\section{References}

Angiotech_Pharmaceuticals. 2002. Micellar paclitaxel does not appear to be effective in patients with multiple sclerosis. Inpharma 1328:11.

Attard G, Greystoke A, Kaye S, De Bono J. 2006. Update on tubulin-binding agents. Pathol Biol (Paris) 54:72-84.

Avasarala JR, Cross AH, Clifford DB, Singer BA, Siegel BA, Abbey EE. 2003. Rapid onset mitoxantrone-induced cardiotoxicity in secondary progressive multiple sclerosis. Mult Scler 9:59-62.

Baranzini SE, Jeong MC, Butunoi C, Murray RS, Bernard CC, Oksenberg JR. 1999. B cell repertoire diversity and clonal expansion in multiple sclerosis brain lesions. J Immunol 163:5133-44.

Beniac DR, Wood DD, Palaniyar N, Ottensmeyer FP, Moscarello MA, Harauz G. 2000. Cryoelectron microscopy of protein-lipid complexes of human myelin basic protein charge isomers differing in degree of citrullination. J Struct Biol 129:80-95.

Bennett JL. 2006. Natalizumab and progressive multifocal leukoencephalopathy: migrating towards safe adhesion molecule therapy in multiple sclerosis. Neurol Res 28:291-8.

Berridge MV, Herst PM, Tan AS. 2005. Tetrazolium dyes as tools in cell biology: New insights into their cellular reduction. Biotechnol Annu Rev 11:127-52.

Bettelli E, Pagany M, Weiner HL, Linington C, Sobel RA, Kuchroo VK. 2003. Myelin oligodendrocyte glycoprotein-specific T cell receptor transgenic mice develop spontaneous autoimmune optic neuritis. J Exp Med 197:1073-81.

Bhalla KN. 2003. Microtubule-targeted anticancer agents and apoptosis. Oncogene 22:9075-86. 
Bogdan C, Ding A. 1992. Taxol, a microtubule-stabilizing antineoplastic agent, induces expression of tumor necrosis factor alpha and interleukin-1 in macrophages. J Leukoc Biol 52:119-21.

Bordeleau ME, Matthews J, Wojnar JM, Lindqvist L, Novac O, Jankowsky E, Sonenberg N, Northcote P, Teesdale-Spittle P, Pelletier J. 2005. Stimulation of mammalian translation initiation factor eIF4A activity by a small molecule inhibitor of eukaryotic translation. Proc Natl Acad Sci USA 102:10460-5.

Brahn E, Tang C, Banquerigo ML. 1994. Regression of collagen-induced arthritis with Taxol, a microtubule stabilizer. Arthritis Rheum 37:839-45.

Broadley SA, Deans J, Sawcer SJ, Clayton D, Compston DA. 2000. Autoimmune disease in first-degree relatives of patients with multiple sclerosis. A UK survey. Brain 123:1102-11.

Brown DL, Little JE, Chaly N, Schweitzer I, Paulin-Levasseur M. 1985. Effects of Taxol on microtubule organization in mouse splenic lymphocytes and on response to mitogenic stimulation. Eur J Cell Biol 37:130-9.

Brunet LR, Finkelman FD, Cheever AW, Kopf MA, Pearce EJ. 1997. IL-4 protects against TNF- $\varepsilon$-mediated cachexia and death during acute schistosomiasis. J Immunol 159:777-85.

Burns J, Rosenzweig A, Zweiman B, Lisak RP. 1983. Isolation of myelin basic protein-reactive T-cell lines from normal human blood. Cell Immunol $81: 435-40$.

Burres NS, Clement JJ. 1989. Antitumor activity and mechanism of action of the novel marine natural products mycalamide- $\mathrm{A}$ and $-\mathrm{B}$ and onnamide. Cancer Res 49:2935-40. 
Byrd-Leifer CA, Block EF, Takeda K, Akira S, Ding A. 2001. The role of MyD88 and TLR4 in the LPS-mimetic activity of Taxol. Eur J Immunol 31:2448-57. Cantorna MT. 2006. Vitamin D and its role in immunology: Multiple sclerosis, and inflammatory bowel disease. Prog Biophys Mol Biol. 92:60-4.

Cao L, Sun D, Cruz T, Moscarello MA, Ludwin SK, Whitaker JN. 2000. Inhibition of experimental allergic encephalomyelitis in the Lewis rat by paclitaxel. $\mathrm{J}$ Neuroimmunol 108:103-11.

Cassidy PB, Moos PJ, Kelly RC, Fitzpatrick FA. 2002. Cyclooxygenase-2 induction by paclitaxel, docetaxel, and taxane analogues in human monocytes and murine macrophages: structure-activity relationships and their implications. Clin Cancer Res 8:846-55.

Cavaletti G, Cavalletti E, Crippa L, Di Luccio E, Oggioni N, Mazzanti B, Biagioli T, Sala F, Sala V, Frigo M and others. 2004. Pixantrone (BBR2778) reduces the severity of experimental allergic encephalomyelitis. J Neuroimmunol 151:5565.

Celada A, Gray PW, Rinderknecht E, Schreiber RD. 1984. Evidence for a $\varepsilon$ interferon receptor that regulates macrophage tumoricidal activity. J Exp Med 160:55-74.

Chavarria A, Alcocer-Varela J. 2004. Is damage in central nervous system due to inflammation? Autoimmun Rev 3:251-60.

Chuang LT, Lotzova E, Heath J, Cook KR, Munkarah A, Morris M, Wharton JT. 1994. Alteration of lymphocyte microtubule assembly, cytotoxicity, and activation by the anticancer drug Taxol. Cancer Res 54:1286-91. 
Confavreux C, Hutchinson M, Hours MM, Cortinovis-Tourniaire P, Moreau T. 1998. Rate of pregnancy-related relapse in multiple sclerosis. Pregnancy in Multiple Sclerosis Group. N Engl J Med 339:285-91.

Ding A, Sanchez E, Nathan CF. 1993. Taxol shares the ability of bacterial lipopolysaccharide to induce tyrosine phosphorylation of microtubuleassociated protein kinase. J Immunol 151:5596-602.

Donaldson KL, Goolsby GL, Wahl AF. 1994. Cytotoxicity of the anticancer agents cisplatin and Taxol during cell proliferation and the cell cycle. Int J Cancer $57: 847-55$.

Dyment DA, Ebers GC, Sadovnick AD. 2004. Genetics of multiple sclerosis. Lancet Neurol 3:104-10.

El Behi M, Dubucquoi S, Lefranc D, Zephir H, De Seze J, Vermersch P, Prin L. 2005. New insights into cell responses involved in experimental autoimmune encephalomyelitis and multiple sclerosis. Immunol Lett 96:11-26.

Elliott JI, Raguz S, Higgins CF. 2004. Multidrug transporter activity in lymphocytes. Br J Pharmacol 143:899-907.

Ercolini AM, Miller SD. 2006. Mechanisms of immunopathology in murine models of central nervous system demyelinating disease. J Immunol 176:3293-8.

Feldmann M. 2002. Development of anti-TNF therapy for rheumatoid arthritis. Nat Rev Immunol 2:364-71.

Feldmann M, Brennan FM, Maini RN. 1996. Role of cytokines in rheumatoid arthritis. Annu Rev Immunol 14:397-440.

Feldmann M, Steinman L. 2005. Design of effective immunotherapy for human autoimmunity. Nature 435:612-9. 
Feofanov A, Charonov S, Fleury F, Kudelina I, Jardillier JC, Nabiev I. 1999.

Confocal spectral imaging analysis of intracellular interactions of mitoxantrone at different phases of the cell cycle. Anticancer Res 19:5341-8.

Fidler JM, DeJoy SQ, Gibbons JJ, Jr. 1986. Selective immunomodulation by the antineoplastic agent mitoxantrone. I. Suppression of B lymphocyte function. J Immunol 137:727-32.

Fuchs DA, Johnson RK. 1978. Cytologic evidence that Taxol, an antineoplastic agent from Taxus brevifolia, acts as a mitotic spindle poison. Cancer Treat Rep 62:1219-22.

Gaitanos TN. 2005. Cellular and molecular actions of peloruside A, a novel anticancer agent. A thesis of Victoria University of Wellington.

Gaitanos TN, Buey RM, Diaz JF, Northcote PT, Teesdale-Spittle P, Andreu JM, Miller JH. 2004. Peloruside A does not bind to the taxoid site on beta-tubulin and retains its activity in multidrug-resistant cell lines. Cancer Res 64:5063-7.

Galvin F, Freeman GJ, Razi-Wolf Z, Benacerraf B, Nadler L, Reiser H. 1993. Effects of cyclosporin A, FK 506, and mycalamide A on the activation of murine CD4+ T cells by the murine B7 antigen. Eur J Immunol 23:283-6.

Gbadamosi J, Buhmann C, Tessmer W, Moench A, Haag F, Heesen C. 2003. Effects of mitoxantrone on multiple sclerosis patients' lymphocyte subpopulations and production of immunoglobulin, TNF- $\varepsilon$ and IL-10. Eur Neurol 49:137-41.

Gelderblom H, Verweij J, van Zomeren DM, Buijs D, Ouwens L, Nooter K, Stoter G, Sparreboom A. 2002. Influence of Cremophor EL on the bioavailability of intraperitoneal paclitaxel. Clin Cancer Res 8:1237-41.

Gligorov J, Lotz JP. 2004. Preclinical pharmacology of the taxanes: implications of the differences. Oncologist 9 (Suppl 2):3-8. 
Gonsette RE. 1996. Mitoxantrone immunotherapy in multiple sclerosis. Mult Scler 1:329-32.

Green LC, Wagner DA, Glogowski J, Skipper PL, Wishnok JS, Tannenbaum SR. 1982. Analysis of nitrate, nitrite, and ${ }^{15} \mathrm{~N}$-nitrate in biological fluids. Anal Biochem 126:131-8.

Hansen MB, Nielsen SE, Berg K. 1989. Re-examination and further development of a precise and rapid dye method for measuring cell growth/cell kill. J Immunol Methods 119:203-10.

Hellings N, Raus J, Stinissen P. 2002. Insights into the immunopathogenesis of multiple sclerosis. Immunol Res 25:27-51.

Helson L, Helson C, Malik S, Ainsworth S, Mangiardi J. 1993. A saturation threshold for Taxol cytotoxicity in human glial and neuroblastoma cells. Anticancer Drugs 4:487-90.

Hickey WF. 1999. Leukocyte traffic in the central nervous system: the participants and their roles. Semin Immunol 11:125-37.

Hofstetter HH, Shive CL, Forsthuber TG. 2002. Pertussis toxin modulates the immune response to neuroantigens injected in incomplete Freund's adjuvant: induction of Th1 cells and experimental autoimmune encephalomyelitis in the presence of high frequencies of Th2 cells. J Immunol 169:117-25.

Hong J, Zang YC, Li S, Rivera VM, Zhang JZ. 2004. Ex vivo detection of myelin basic protein-reactive T cells in multiple sclerosis and controls using specific TCR oligonucleotide probes. Eur J Immunol 34:870-81. 
Hood KA. 2002. Biological activity of Mycale marine sponge secondary metabolites, including peloruside $\mathrm{A}$, a novel microtubule stabilising compound. A thesis of Victoria University of Wellington.

Hood KA, Bäckström BT, West LM, Northcote PT, Berridge MV, Miller JH. 2001. The novel cytotoxic sponge metabolite peloruside A, structurally similar to bryostatin-1, has unique bioactivity independent of protein kinase C. Anticancer Drug Des 16:155-66.

Hood KA, West LM, Rouwe B, Northcote PT, Berridge MV, Wakefield SJ, Miller JH. 2002. Peloruside A, a novel antimitotic agent with paclitaxel-like microtubule-stabilizing activity. Cancer Res 62:3356-60.

Horwitz SB, Cohen D, Rao S, Ringel I, Shen HJ, Yang CP. 1993. Taxol: mechanisms of action and resistance. J Natl Cancer Inst Monogr 15:55-61. Jimenez-Barbero J, Canales A, Northcote PT, Buey RM, Andreu JM, Diaz JF. 2006. NMR determination of the bioactive conformation of peloruside A bound to microtubules. J Am Chem Soc 128:8757-65.

Johnson KP, Brooks BR, Cohen JA, Ford CC, Goldstein J, Lisak RP, Myers LW, Panitch HS, Rose JW, Schiffer RB. 1995. Copolymer 1 reduces relapse rate and improves disability in relapsing-remitting multiple sclerosis: results of a phase III multicenter, double-blind placebo-controlled trial. The Copolymer 1 Multiple Sclerosis Study Group. Neurology 45:1268-76.

Joo HG. 2003. Altered maturation of dendritic cells by Taxol, an anticancer drug. J Vet Sci 4:229-34.

Jordan M, Toso R, Thrower D, Wilson L. 1993. Mechanism of mitotic block and inhibition of cell proliferation by Taxol at low concentrations. Proc Natl Acad Sci USA 90:9552-9556. 
Juedes AE, Hjelmstrom P, Bergman CM, Neild AL, Ruddle NH. 2000. Kinetics and cellular origin of cytokines in the central nervous system: insight into mechanisms of myelin oligodendrocyte glycoprotein-induced experimental autoimmune encephalomyelitis. J Immunol 164:419-26.

Kabat EA, Freedman DA, Murray JP, Knaub V. 1950. A study of the crystalline albumin, gamma globulin and total protein in the cerebrospinal fluid of 100 cases of multiple sclerosis and in other diseases. Am J Med Sci 219:55-64.

Karpus WJ, Ransohoff RM. 1998. Chemokine regulation of experimental autoimmune encephalomyelitis: temporal and spatial expression patterns govern disease pathogenesis. J Immunol 161:2667-71.

Kerfoot SM, Long EM, Hickey MJ, Andonegui G, Lapointe BM, Zanardo RC, Bonder C, James WG, Robbins SM, Kubes P. 2004. TLR4 contributes to disease-inducing mechanisms resulting in central nervous system autoimmune disease. J Immunol 173:7070-7.

Kim SC, Kim DW, Shim YH, Bang JS, Oh HS, Wan Kim S, Seo MH. 2001. In vivo evaluation of polymeric micellar paclitaxel formulation: toxicity and efficacy. J Control Release 72:191-202.

Kirikae T, Ojima I, Kirikae F, Ma Z, Kuduk SD, Slater JC, Takeuchi CS, Bounaud PY, Nakano M. 1996. Structural requirements of taxoids for nitric oxide and tumor necrosis factor production by murine macrophages. Biochem Biophys Res Commun 227:227-35.

Kotsakis A, Sarra E, Peraki M, Koukourakis M, Apostolaki S, Souglakos J, Mavromanomakis E, Vlachonikolis J, Georgoulias V. 2000. Docetaxelinduced lymphopenia in patients with solid tumors: a prospective phenotypic analysis. Cancer 89:1380-6. 
La Flamme AC, Ruddenklau K, Backstrom BT. 2003. Schistosomiasis decreases central nervous system inflammation and alters the progression of experimental autoimmune encephalomyelitis. Infect Immun 71(9):4996-5004.

Lee JM, Olitsky PK. 1955. Simple method for enhancing development of acute disseminated encephalomyelitis in mice. Proc Soc Exp Biol Med 89:263-6.

Lee M, Yea SS, Jeon YJ. 2000. Paclitaxel causes mouse splenic lymphocytes to a state hyporesponsive to lipopolysaccharide stimulation. Int J Immunopharmacol 22:615-21.

Loke P, Allison JP. 2003. PD-L1 and PD-L2 are differentially regulated by Th1 and Th2 cells. Proc Natl Acad Sci USA 100:5336-41.

Lucchinetti C, Bruck W, Parisi J, Scheithauer B, Rodriguez M, Lassmann H. 2000. Heterogeneity of multiple sclerosis lesions: implications for the pathogenesis of demyelination. Ann Neurol 47:707-17.

Lyons MJ, Amador R, Petito C, Nagashima K, Weinreb H, Zabriskie JB. 1986. Inhibition of acute experimental allergic encephalomyelitis in mice by colchicine. J Exp Med 164:1803-8.

Maher JM, Slitt AL, Cherrington NJ, Cheng X, Klaassen CD. 2005. Tissue distribution and hepatic and renal ontogeny of the multidrug resistanceassociated protein (Mrp) family in mice. Drug Metab Dispos 33:947-55.

Mamula MJ, Gee RJ, Elliott JI, Sette A, Southwood S, Jones PJ, Blier PR. 1999. Isoaspartyl post-translational modification triggers autoimmune responses to self-proteins. J Biol Chem 274:22321-7.

Manfredi JJ, Parness J, Horwitz SB. 1982. Taxol binds to cellular microtubules. J Cell Biol 94:688-96. 
Manthey CL, Perera PY, Salkowski CA, Vogel SN. 1994. Taxol provides a second signal for murine macrophage tumoricidal activity. J Immunol 152:825-31.

Marrie RA. 2004. Environmental risk factors in multiple sclerosis aetiology. Lancet Neurol 3:709-18.

Martyn CN, Cruddas M, Compston DA. 1993. Symptomatic Epstein-Barr virus infection and multiple sclerosis. J Neurol Neurosurg Psychiatry 56:167-68.

McFarland HF. 1992. Twin studies and multiple sclerosis. Ann Neurol 32:722-3.

McGeachy MJ, Stephens LA, Anderton SM. 2005. Natural recovery and protection from autoimmune encephalomyelitis: contribution of CD4+CD25+ regulatory cells within the central nervous system. J Immunol 175:3025-32.

Mekala DJ, Alli RS, Geiger TL. 2005. IL-10-dependent infectious tolerance after the treatment of experimental allergic encephalomyelitis with redirected CD4+CD25+ T lymphocytes. Proc Natl Acad Sci USA 102:11817-22.

Miller JH, Rouwe B, Gaitanos TN, Hood KA, Crume KP, Bäckström BT, La Flamme AC, Berridge MV, Northcote PT. 2004. Peloruside A enhances apoptosis in $\mathrm{H}$ - ras -transformed cells and is cytotoxic to proliferating $\mathrm{T}$ cells. Apoptosis 9:785-796.

Miller SD, McMahon EJ, Schreiner B, Bailey SL. 2007. Antigen presentation in the CNS by myeloid dendritic cells drives progression of relapsing experimental autoimmune encephalomyelitis. Ann N Y Acad Sci 1103:179-91.

Moore FG, Wolfson C. 2002. Human herpes virus 6 and multiple sclerosis. Acta Neurol Scand 106:63-83.

Mosmann T. 1983. Rapid colorimetric assay for cellular growth and survival: application to proliferation and cytotoxicity assays. J Immunol Methods 65:55-63. 
Muhlradt PF, Sasse F. 1997. Epothilone B stabilizes microtubuli of macrophages like Taxol without showing Taxol-like endotoxin activity. Cancer Res 57:3344-6. Mullins DW, Alleva DG, Burger CJ, Elgert KD. 1997. Taxol, a microtubulestabilizing antineoplastic agent, differentially regulates normal and tumorbearing host macrophage nitric oxide production. Immunopharmacology $37: 63-73$.

Mullins DW, Burger CJ, Elgert KD. 1998a. Tumor growth modulates macrophage nitric oxide production following paclitaxel administration. Int $\mathrm{J}$ Immunopharmacol 20:537-51.

Mullins DW, Koci MD, Burger CJ, Elgert KD. 1998b. Interleukin-12 overcomes paclitaxel-mediated suppression of T-cell proliferation. Immunopharmacol Immunotoxicol 20:473-92.

Munger KL, Zhang SM, O'Reilly E, Hernan MA, Olek MJ, Willett WC, Ascherio A. 2004. Vitamin D intake and incidence of multiple sclerosis. Neurology 62:605.

Muraro PA, Wandinger KP, Bielekova B, Gran B, Marques A, Utz U, McFarland HF, Jacobson S, Martin R. 2003. Molecular tracking of antigen-specific T cell clones in neurological immune-mediated disorders. Brain 126:20-31.

Nakashima H, Tasaki A, Kubo M, Kuroki H, Matsumoto K, Tanaka M, Nakamura M, Morisaki T, Katano M. 2005. Effects of docetaxel on antigen presentation-related functions of human monocyte-derived dendritic cells. Cancer Chemother Pharmacol 55:479-87.

Ohmori K, Hong Y, Fujiwara M, Matsumoto Y. 1992. In situ demonstration of proliferating cells in the rat central nervous system during experimental 
autoimmune encephalomyelitis. Evidence suggesting that most infiltrating $\mathrm{T}$ cells do not proliferate in the target organ. Lab Invest 66:54-62.

Ojima I, Fumero-Oderda CL, Kuduk SD, Ma Z, Kirikae F, Kirikae T. 2003. Structure-activity relationship study of taxoids for their ability to activate murine macrophages as well as inhibit the growth of macrophage-like cells. Bioorg Med Chem 11:2867-88.

Oliver SJ, Banquerigo ML, Brahn E. 1994. Suppression of collagen-induced arthritis using an angiogenesis inhibitor, AGM-1470, and a microtubule stabilizer, Taxol. Cell Immunol 157:291-9.

Peachman KK, Rao M, Palmer DR, Zidanic M, Sun W, Alving CR, Rothwell SW. 2004. Functional microtubules are required for antigen processing by macrophages and dendritic cells. Immunol Lett 95:13-24.

Pellman D. 2001. Cancer. A CINtillating new job for the APC tumor suppressor. Science 291:2555-6.

Perera PY, Qureshi N, Vogel SN. 1996. Paclitaxel (Taxol)-induced NF-kappaB translocation in murine macrophages. Infect Immun 64:878-84.

Pineda O, Farras J, Maccari L, Manetti F, Botta M, Vilarrasa J. 2004. Computational comparison of microtubule-stabilising agents laulimalide and peloruside with Taxol and colchicine. Bioorg Med Chem Lett 14:4825-9.

Planczyk MJ, Hopke C, Vandenbark AA, Offner H. 2006. Estrogen-mediated immunomodulation involves reduced activation of effector T cells, potentiation of Treg cells, and enhanced expression of the PD-1 costimulatory pathway. J Neurosci Res 84(2):370-8. 
Ponsonby AL, McMichael A, van der Mei I. 2002. Ultraviolet radiation and autoimmune disease: insights from epidemiological research. Toxicology 181-182:71-8.

Price KS, Castells MC. 2002. Taxol reactions. Allergy Asthma Proc 23:205-8.

Raijmakers R, Vogelzangs J, Croxford JL, Wesseling P, van Venrooij WJ, Pruijn GJ. 2005. Citrullination of central nervous system proteins during the development of experimental autoimmune encephalomyelitis. J Comp Neurol 486:243-53.

Rao P, Falk L, Dougherty S, Sawada T, Pluznik D. 1997. Colchicine down-regulates lipopolysaccharide-induced granulocyte-macrophage colony-stimulating factor production in murine macrophages. J Immunol 159:3531-3539.

Richaud-Patin Y, Soto-Vega E, Jakez-Ocampo J, Llorente L. 2004. P-glycoprotein in autoimmune diseases. Autoimmun Rev 3:188-92.

Ridge SC, Sloboda AE, McReynolds RA, Levine S, Oronsky AL, Kerwar SS. 1985. Suppression of experimental allergic encephalomyelitis by mitoxantrone. Clin Immunol Immunopathol 35:35-42.

Rivers T, Schwentker F. 1935. Encephalomyelitis accompanied by myelin destruction experimentally produced in monkeys. J Exp Med 61:689-701.

Romo D. 1998. Total synthesis and immunosuppressive activity of (-)-pateamine A and related compounds: implementaion of a $\varepsilon$-lactam-based macrocyclization. J Am Chem Soc 120:12237-12254.

Romo D, Choi NS, Li S, Buchler I, Shi Z, Liu JO. 2004. Evidence for separate binding and scaffolding domains in the immunosuppressive and antitumor marine natural product, pateamine a: design, synthesis, and activity studies leading to a potent simplified derivative. J Am Chem Soc 126:10582-8. 
Rowinsky EK, Donehower RC, Jones RJ, Tucker RW. 1988. Microtubule changes and cytotoxicity in leukemic cell lines treated with Taxol. Cancer Res 48:4093-100.

Sakaguchi S, Sakaguchi N. 2005. Animal models of arthritis caused by systemic alteration of the immune system. Curr Opin Immunol 17:589-94.

Sako T, Burioka N, Yasuda K, Tomita K, Miyata M, Kurai J, Chikumi H, Watanabe M, Suyama H, Fukuoka Y. 2004. Cellular immune profile in patients with non-small cell lung cancer after weekly paclitaxel therapy. Acta Oncol 43:159.

Schiff PB, Fant J, Horwitz SB. 1979. Promotion of microtubule assembly in vitro by Taxol. Nature 277:665-7.

Schiff PB, Horwitz SB. 1980. Taxol stabilizes microtubules in mouse fibroblast cells. Proc Natl Acad Sci USA 77:1561-5.

Segal BM, Chang JT, Shevach EM. 2000. CpG oligonucleotides are potent adjuvants for the activation of autoreactive encephalitogenic T cells in vivo. J Immunol 164:5683-8.

Sewell D, Qing Z, Reinke E, Elliot D, Weinstock J, Sandor M, Fabry Z. 2003. Immunomodulation of experimental autoimmune encephalomyelitis by helminth ova immunization. Int Immunol 15(1):59-69.

Schmied M, Duda PW, Krieger JI, Trollmo C, Hafler DA. 2003. In vitro evidence that subcutaneous administration of glatiramer acetate induces hyporesponsive T cells in patients with multiple sclerosis. Clin Immunol 106(3):163-74. 
Sicotte NL, Liva SM, Klutch R, Pfeiffer P, Bouvier S, et al. 2002. Treatment of multiple sclerosis with the pregnancy hormone estriol. Ann. Neurol. 52:42128

Skundric DS. 2005. Experimental models of relapsing-remitting multiple sclerosis: current concepts and perspective. Curr Neurovasc Res 2:349-62.

Somerset DA, Zhang Y, Kilby MD, Samsom DM, Drayson MT. 2004. Normal human pregnancy is associated with an elevation in the immune suppressive CD25+CD4+ regulatory T-cell subset. Immunology 112(1);38-43.

Sospedra M, Martin R. 2005. Immunology of multiple sclerosis. Annu Rev Immunol 23:683-747.

Sriram S, Mitchell W, Stratton C. 1998. Multiple sclerosis associated with Chlamydia pneumoniae infection of the CNS. Neurology 50:571-72.

Sriram S, Steiner I. 2005. Experimental allergic encephalomyelitis: A misleading model of multiple sclerosis. Ann Neurol 58:939-45.

Steinman L. 1999. Assessment of animal models for MS and demyelinating disease in the design of rational therapy. Neuron 24:511-4.

Steinman L, Zamvil SS. 2005. Virtues and pitfalls of EAE for the development of therapies for multiple sclerosis. Trends Immunol 26:565-71.

Stinissen P, Raus J, Zhang J. 1997. Autoimmune pathogenesis of multiple sclerosis: role of autoreactive T lymphocytes and new immunotherapeutic strategies. Crit Rev Immunol 17:33-75.

Sultzer BM. 1968. Genetic control of leucocyte responses to endotoxin. Nature 219:1253-4.

Targoni OS, Baus J, Hofstetter HH, Hesse MD, Karulin AY, Boehm BO, Forsthuber TG, Lehmann PV. 2001. Frequencies of neuroantigen-specific T cells in the 
central nervous system versus the immune periphery during the course of experimental allergic encephalomyelitis. J Immunol 166:4757-64.

Tsavaris N, Kosmas C, Vadiaka M, Kanelopoulos P, Boulamatsis D. 2002. Immune changes in patients with advanced breast cancer undergoing chemotherapy with taxanes. Br J Cancer 87:21-7.

Ubogu EE, Cossoy MB, Ransohoff RM. 2005. The expression and function of chemokines involved in CNS inflammation. Trends Pharmacol Sci 27:48-55.

Van Boekel MA, van Venrooij WJ. 2003. Modifications of arginines and their role in autoimmunity. Autoimmun Rev 2:57-62.

Van Amerongen BM, Dijkstra CD, Lips P, Polman CH. 2004. Multiple sclerosis and vitamin D: an update. Eur J Clin Nutr 58:1095-109.

Viglietta V, Baecher-Allan C, Weiner HL, Hafler DA. 2004. Loss of functional suppression by CD4+CD25+ regulatory $\mathrm{T}$ cells in patients with multiple sclerosis. J Exp Med 199:971-9.

Vila-Del Sol V, Diaz-Munoz MD, Fresno M. 2006. Requirement of tumor necrosis factor $\{$ alpha $\}$ and nuclear factor- $\{$ kappa $\}$ B in the induction by IFN\{gamma\} of inducible nitric oxide synthase (iNOS) in macrophages. $\mathbf{J}$ Leukoc Biol. Oct 11 [Epub ahead of print].

Visser L, Jan de Heer H, Boven LA, van Riel D, van Meurs M, Melief MJ, Zahringer U, van Strijp J, Lambrecht BN, Nieuwenhuis EE. 2005. Proinflammatory bacterial peptidoglycan as a cofactor for the development of central nervous system autoimmune disease. J Immunol 174:808-16.

Von Andrian UH, Engelhardt B. 2003. Alpha4 integrins as therapeutic targets in autoimmune disease. N Engl J Med 348:68-72. 
Walsh MJ, Tourtellotte WW. 1986. Temporal invariance and clonal uniformity of brain and cerebrospinal $\operatorname{IgG}$, IgA, and IgM in multiple sclerosis. J Exp Med $163: 41-53$.

Walsh MJ, Tourtellotte WW, Shapshak P. 1986. Immunoglobulin heavy chain associated protein in multiple sclerosis cerebrospinal fluid. Mol Immunol 23:1117-23.

Wandinger KP, Jabs W, Siekhaus A, Bubel S, Trillenberg P, et al. 2000. Association between clinical disease activity and Epstein-Barr virus reactivation in MS Neurology 55:178-84.

Wani MC, Taylor HL, Wall ME, Coggon P, McPhail AT. 1971. Plant antitumor agents. VI. The isolation and structure of Taxol, a novel antileukemic and antitumor agent from Taxus brevifolia. J Am Chem Soc 93:2325-7.

Wekerle H, Linington C. 2006. Organ specific autoantigens and the autoreactiveT cell repertoire: the case of myelin oligodendrocyte glycoprotein. Eur J Immunol 36:512-5.

West LM, Northcote PT, Battershill CN. 2000. Peloruside A: a potent cytotoxic macrolide isolated from the New Zealand marine sponge Mycale sp. J Org Chem 65:445-9.

Wingerchuk DM, Lesaux J, Rice GP, Kremenchutzky M, Ebers GC. 2005. A pilot study of oral calcitriol (1,25-dihydroxyvitamin D3) for relapsing-remitting multiple sclerosis. J Neurol Neurosurg Psychiatry 76(9):1294-6.

Yong T, Meininger GA, Linthicum DS. 1993. Enhancement of histamine-induced vascular leakage by pertussis toxin in SJL/J mice but not BALB/c mice. $\mathrm{J}$ Neuroimmunol 45:47-52. 
Yong VW. 2002. Differential mechanisms of action of interferon- $\varepsilon$ and glatiramer aetate in MS. Neurology 59:802-8.

Yuan JH, Zhang RP, Zhang RG, Guo LX, Wang XW, Luo D, Xie Y, Xie H. 2000. Growth-inhibiting effects of Taxol on human liver cancer in vitro and in nude mice. World J Gastroenterol 6:210-215.

Zhang AL, Russell PJ. 2006. Paclitaxel suppresses the growth of primary prostate tumours (RM-1) and metastases in the lung in C57BL/6 mice. Cancer Lett 233:185-91

Zhang X, Koldzic DN, Izikson L, Reddy J, Nazareno RF, Sakaguchi S, Kuchroo VK, Weiner HL. 2004. IL-10 is involved in the suppression of experimental autoimmune encephalomyelitis by $\mathrm{CD} 25+\mathrm{CD} 4+$ regulatory $\mathrm{T}$ cells. Int Immunol 16:249-56. 


\section{Appendix A: Buffers and Reagents}

\section{Cell Wash}

Dulbecco's Modified Eagle Medium containing:

- $1 \%$ pen-strep (100 U penicillin/ml and 100\&g streptomycin/ml)

- 30 mM HEPES

\section{CTCM}

Dulbecco's Modified Eagle Medium containing:

- 10\% Foetal Calf Serum (FCS)

- $1 \%$ pen-strep (100 U penicillin/ml and 100\&g streptomycin/ml)

- 1 mM L-glutamine

- 10 mM HEPES

- $5.5 \mu \mathrm{M} \varepsilon$-mercaptoethanol

ELISA Capture Buffer (IFN-a, IL-12, and IL-10)

- $0.1 \mathrm{M} \mathrm{Na}_{2} \mathrm{HPO}_{4}$

- $\mathrm{ddH}_{2} \mathrm{O}$

$\mathrm{pH}$ to 9.0

ELISA Capture Buffer (TNF- $\alpha$ )

- $0.1 \mathrm{M} \mathrm{Na}_{2} \mathrm{HPO}_{4}$

- $\mathrm{ddH}_{2} \mathrm{O}$

$\mathrm{pH}$ to $\mathbf{6 . 0}$ 


\section{ELISA Development-Stopping Reagent}

- $0.18 \mathrm{M} \mathrm{H}_{2} \mathrm{SO}_{4}$

- $\mathrm{ddd}_{2} \mathrm{O}$

\section{FACS Buffer}

$-0.1 \%$ sodium azide

- $2 \%$ foetal calf serum

- PBS

\section{MTT Solution}

- sterile $10 \mathrm{mM}$ dPBS

- $5 \mathrm{mg} / \mathrm{ml}$ MTT (Sigma)

\section{MTT Solubiliser $(500 \mathrm{ml})$}

- $10 \%(50 \mathrm{~g})$ sodium dodecyl sulfate (SDS)

- $45 \%$ (225 ml) dimethylformamide (DMF)

pH 4.5 with acetic acid

Diluted to $500 \mathrm{ml}$ with $\mathrm{ddH}_{2} \mathrm{O}$

\section{PBS}

Sterile $\mathrm{ddH}_{2} \mathrm{O}$ containing:
- $\mathrm{Na}_{2} \mathrm{HPO}_{4}$
$8.7 \mathrm{mM}$
- $\mathrm{NaH}_{2} \mathrm{PO}_{4}$
$1.3 \mathrm{mM}$
$-\mathrm{NaCl}$
$145 \mathrm{mM}$ 
pH 7.4

\section{Pertussis Toxin Buffer}

Sterile $\mathrm{ddH}_{2} \mathrm{O}$ containing:

- Tris-Cl $\quad 15 \mathrm{mM}$

$\begin{array}{ll}-\mathrm{NaCl} & 0.5 \mathrm{M}\end{array}$

- Triton X-100 $\quad 0.017 \%$

\section{TPBS}

- PBS containing $0.05 \%$ Tween-20

\section{Tris Buffer (0.1 M; 1 Litre)}

- Tris Base $\quad 12.1 \mathrm{~g}$

- 1N HCL $\quad 81.5 \mathrm{ml}$

- Distilled water $900 \mathrm{ml}$

$\mathrm{pH}$ to 7.4

\section{Zinc Fixative (1 Litre)}

- Calcium Acetate $0.5 \mathrm{~g}$

- Zinc Acetate $5 \mathrm{~g}$

- Zinc Chloride $5 \mathrm{~g}$

- 0.1 M Tris Buffer $1 \mathrm{~L}$ 


\section{Appendix B: Determining peloruside purity}

Peloruside is a novel compound with a very limited supply. The purity of peloruside was questioned when it was observed in our lab that non-proliferating M $\varnothing$ died when cultured with peloruside, which is known to be cytotoxic to proliferating cells only. Because of this, there are potential issues regarding the purity of the peloruside used in the present experiment. Using nuclear magnetic resonance (NMR), the purity of peloruside was determined by the Natural Products Group from the School of Chemical and Physical Sciences, Victoria University of Wellington. There were two stocks of peloruside that were used throughout this thesis - an older batch with uncertain purity, and a newer, purer batch as determined by NMR spectra. These two batches were tested side-by-side in BMM $\varnothing$ and Con-A stimulated splenocytes to investigate possible cytotoxic contaminants. The newer batch of peloruside had little effect on non-proliferating BMM $\varnothing$ as determined by the MTT assay after a $72 \mathrm{hr}$ culture (Panel a). The older batch of peloruside completely abolished metabolic activity at concentrations as low as $31 \mathrm{nM}$ under the same culture conditions. With concentrations at or above $31 \mathrm{nM}$, blebbing and shriveling of BMM $\varnothing$, induced by the older batch of peloruside, was evident within hours, and clearly visible under a light microscope (no pictures available). Both Con-A stimulated (Panel b) and unstimulated splenocytes (Panel c) were susceptible to the cytotoxicity of the older peloruside at similar concentrations; whereas, the newer peloruside was only cytotoxic to proliferating splenocytes (Panel b), albeit only partially in this experiment. This set of experiments was performed once to quickly verify the quality of the peloruside. Unless it is specifically stated, all work with peloruside was performed using the newer batch. 


\section{a. $\quad$ BMMØ}

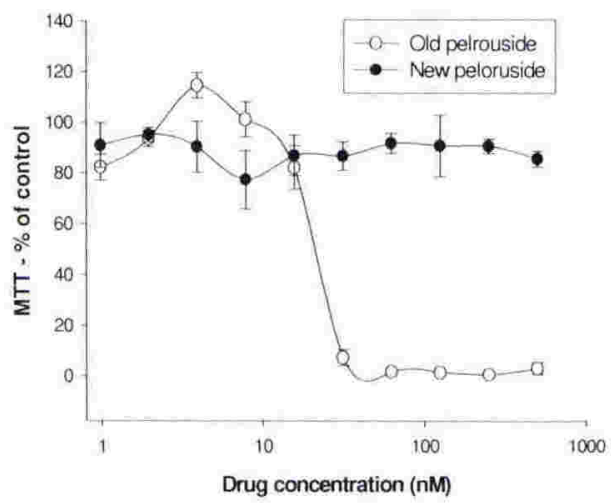

b. Stimulated Splenocytes

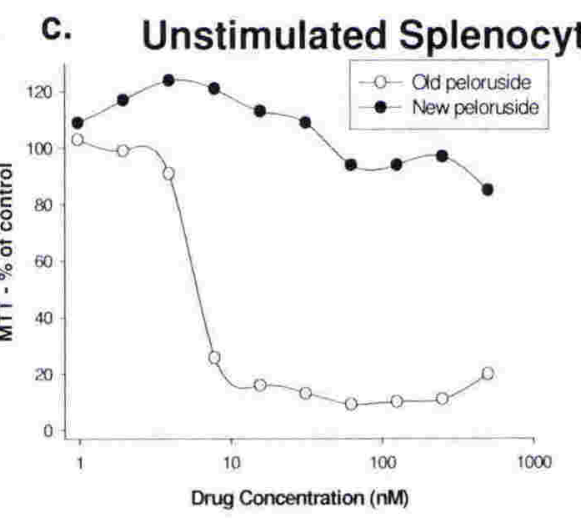

Testing the relative purity of a newer batch vs. an older batch of peloruside. The older batch of peloruside (open circles) strongly inhibited BMM $\varnothing$ metabolism (a) and was cytotoxic to stimulated splenocytes (b) and unstimulated splenocytes (c). The newer peloruside (filled circles) had little or no effect in BMMØ (a) and unstimulated splenocytes (c). Mild cytotoxicity was observed in stimulated splenocytes (b). Tests were performed once in triplicate. Results are expressed as a \% of an untreated control. 


\title{
Appendix C: FACS Gating
}

\author{
Annexin/PI staining
}

a.

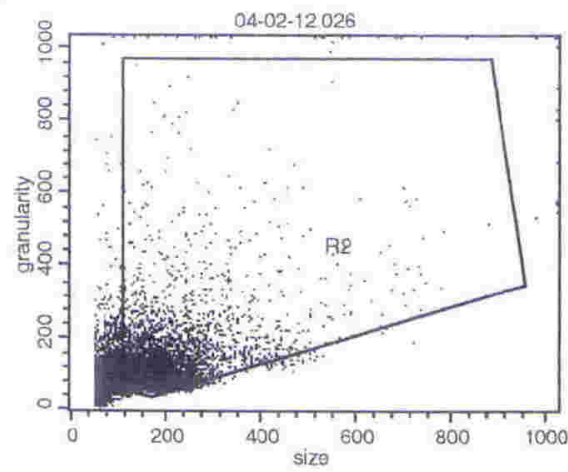

c.

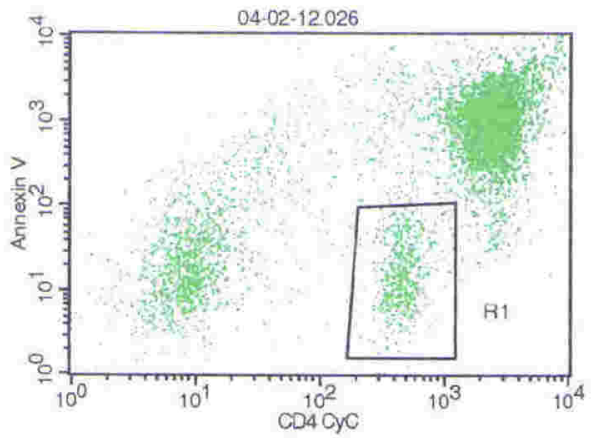

b.

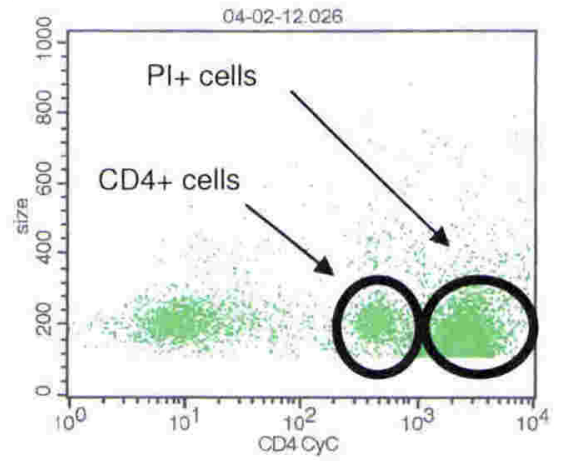

d.

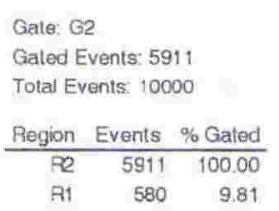

Determining T cell apoptosis by FACS

Splenocytes were cultured with anti-CD3 and cytotoxic agents (Chapter 3.3.1) for 24 or $72 \mathrm{hr}$. Cells were stained with markers for CD4+ or CD8+ T cells, and Annexin V/PI. The sample represented above (Panels a-d) is from a 24-hr culture containing pateamine at concentrations slightly above the $\mathrm{IC}_{50}$ value, and is stained with Annexin V/PI and a CD4+ marker. The emission spectrum of $\mathrm{PI}$ encompasses the FL-2 and FL-3 fluorescent channels, causing $\mathrm{PI}$-stained events to appear in the same detection channel as the $\mathrm{CD} 4+$ and CD8+ labels (FL-3), which are easily discernable based on the level of fluorescence (Panel b). Back-gating in Panel (b) determined where to set the gates for R2 in Panel (a), which excludes non-cellular events and debris. Panels (b) \& (c) show events that occur within R2 from Panel (a). The $\%$ of CD4+ or CD8+ non-apoptotic cells was determined by placing a gate (R1) around the Annexin V/PI negative cells and CD4+ or CD8+ cells (Panel C). The number of events in R1 in the representative sample above is 9.81 (Panel d; under the "\% Gated" in R1). This number was determined for all samples, including a control sample from the drug-free culture. To determine the comparative percentages of non-apoptotic cells, the "\% Gated" value for R1 in each sample was divided by the "\% Gated" R1 value from the drügfree control. 
a.

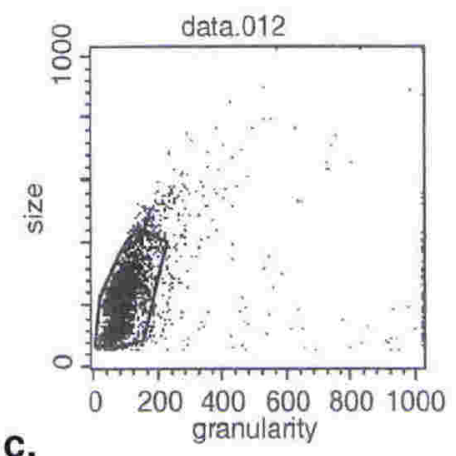

c.

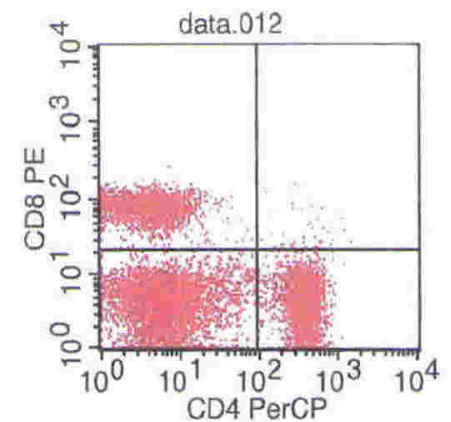

b.

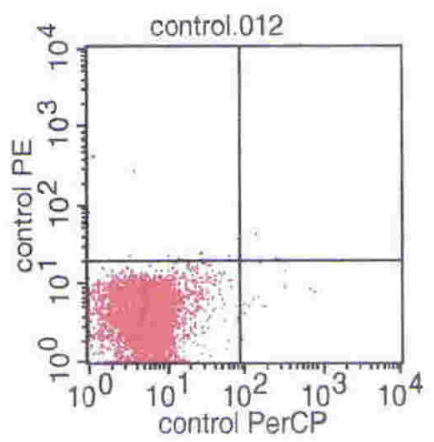

d.

Gate: G1

Gated Events: 9579

Total Events: 10000

$X$ Parameter: CD4 PerCP (Log)

Y Parameter: CD8 PE (Log)

Quad Location: 94,21

\begin{tabular}{|c|c|c|c|}
\hline zuad & Events & Gate & To \\
\hline UL & 2111 & 22.04 & 21. \\
\hline UR & 37 & 0.39 & 0.37 \\
\hline LL & 4860 & 50.74 & 48.60 \\
\hline LR & 2571 & 26.84 & 25. \\
\hline
\end{tabular}

Identifying percentages of lymphocyte sub-populations from LN cells.

Cells Isolated from LN were labeled with marker antibodies and analysed

by FACS. Shown above is a representative sample of the $L N$ cell population identification process. Panel (a) shows the gate (black line around the accumulation of events) placed around the region in which the vast majority of lymphocytes are detected. Panels $(b \& c)$ show the events that occur after the gating in Panel (a). Quadrants settings were determined in a sample stained with fluorescently-labeled control IgG antibodies to compensate for autofluorescence (b). Panel (c) shows the distinct lymphocyte sub-populations within the previously set quadrant from (a). The statistics obtained from this sample are shown in Panel (d), where the lymphocyte sub-populations were calculated based on the "\% Gated" for the specific cell sub-type in each quadrant. Lymphocyte populations from each sample were averaged, and were represented in each experiment as \pm SEM . 


\section{Identifying proliferation in $\mathrm{CD}^{+} \mathrm{T}$ cells from CFSE-labeled $2 \mathrm{D} 2$ cells}

a.

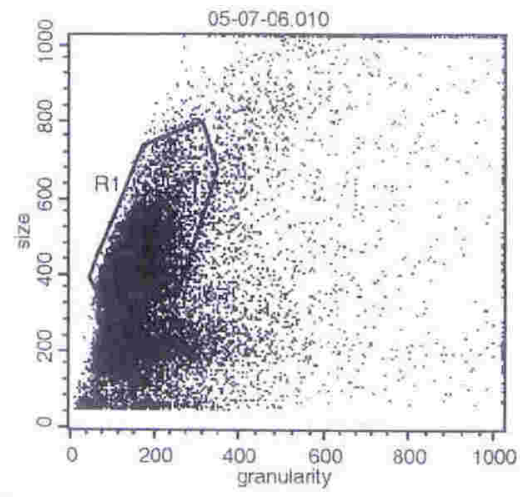

c.

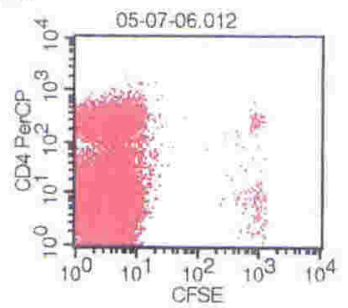

d.

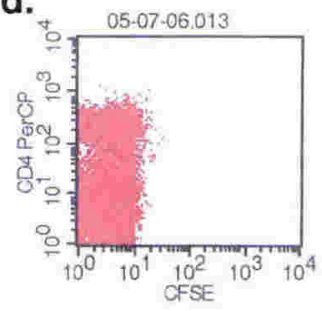

b.

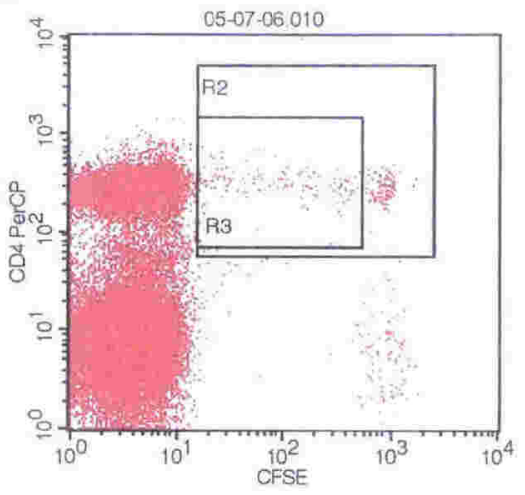

e.

$$
\begin{aligned}
& \text { File: } 05-07-06.010 \\
& \text { Gate: G1 } \\
& \text { Total Events: } 50000 \\
& \begin{array}{cr}
\text { Region } \% \text { Gated } \\
\hline \text { R1 } & 100.00 \\
\text { P2 } & 0.70 \\
\text { R3 } & 0.41
\end{array}
\end{aligned}
$$

\section{Determining the extent of in vivo proliferation of CFSE-labeled cells from}

2D2 mice.

Splenocyte and LN cells from 2D2 mice, which are transgenic to have over $80 \%$ of CD4+ T cells expressing MOG-specific TCR, were transferred to wild-type $\mathrm{C} 57 \mathrm{BL} / 6$ mice. These animals were MOG-immunised and either treated with Taxol, or left untreated. The above sample (Panels a \& b) represents a typical FACS plot from this set of experiments. Two other control groups were included: 1) a non-immunised/untreated group that received CFSE labeled cells (Panel c) and 2) a non-immunised/untreated group that did not receive CFSE-labeled cells (Panel d). After 4-5 days p.i., the DLN cells were removed from each group and labeled with a CD4+ fluorescent marker. The gate in Panel (a) (R1) surrounds a dense region of events that contained the majority of CD4 and CFSE doublepositive events, as determined by back-gating. To determine the $\%$ of proliferating CD4+CFSE+ cells, the R2 gate was placed around ALL of the CD4+CFSE+ cells, and the R3 gate was placed around only the CD4+CFSE+ cells that have undergone one cell division, as determined by the shift in fluorescence (Panel b). The R3 value was divided by the R2 value (Panel e) in order to calculate $\%$ of proliferating MOG-specific CD4+ cells. Panel (c) shows the position of nonproliferating CD4+CFSE+ cells from non-immunised mice that received CFSElabeled cells, and Panel (d) shows the position of cells from a normal control mouse. 